Supporting Information

for

\title{
Enantioselective Catalytic Ring Expansion of Methylenecyclopropyl Amides Promoted by a Chiral Magnesium Lewis Acid
}

\author{
Catherine Taillier and Mark Lautens* \\ Davenport Research Laboratories, Department of Chemistry, University of Toronto, \\ 80 St. George Street, Toronto, Ontario, M5S 3H6, Canada \\ mlautens@chem.utoronto.ca
}

Table of contents

Page

I. General experimental details $\quad S 1$

$\begin{array}{ll}\text { II. Preparation of starting materials } & S 2\end{array}$

III. Enantioselective Ring Expansion of Methylenecyclopropyl Amides $\quad S 4$

IV. Determination of the absolute configuration of methylenepyrrolidine 8i $\quad S 11$

V. ${ }^{1} \mathrm{H}$ and ${ }^{13} \mathrm{C}-\mathrm{NMR}$ spectra of ring-expanded products $\quad S 13$

$\begin{array}{ll}\text { VI. HPLCs of ring-expanded products } & \text { S29 }\end{array}$

\section{General experimental details}

All flasks were flame-dried under a stream of argon and cooled before use. All experiments were performed under anhydrous conditions under an atmosphere of argon. Solvents and solutions were transferred with syringes and cannulae using standard inert atmosphere techniques. Tetrahydrofuran (THF) was distilled under nitrogen from $\mathrm{Na}$ /benzophenone immediately prior to use. Dichloromethane was distilled under nitrogen from $\mathrm{CaH}_{2}$ immediately before use. All reagents were used as received from Sigma-Aldrich unless otherwise indicated. $\mathrm{MgI}_{2}$ was weighed out in a glovebox to prevent absorption of water. Column chromatography was carried out as "flash chromatography" as reported by Still using neutral silica (Silicycle, Quebec, Canada). ${ }^{1}$

Melting points were recorded using a Fisher-Johns melting point apparatus and are uncorrected. ${ }^{1} \mathrm{H}$, and ${ }^{13} \mathrm{C}$ NMR spectra were recorded using either a Varian Gemini 300 or Varian Unity $400 \mathrm{MHz}$ spectrometers in $\mathrm{CDCl}_{3}$ with chemical shifts relative to tetramethylsilane (TMS, $0 \mathrm{ppm}$ ) unless otherwise reported. Spectral features are tabulated in the following order: chemical shift $(\delta, \mathrm{ppm})$; multiplicity (s-singlet, d-doublet, t-triplet, q-quartet, m-complex multiplet); number of protons; coupling constants $(\mathrm{J}, \mathrm{Hz})$. No special notation is used for equivalent carbons. IR spectra were obtained using a Nicolet DX FT IR spectrometer as thin films on $\mathrm{NaCl}$ plates. Optical rotations were obtained using a Perkin-Elmer 243 B polarimeter. High-resolution mass spectra were obtained using a VG 70-250S (double focusing) mass spectrometer at $70 \mathrm{eV}$. Enantiomeric excess was determined on an analytical HPLC using Chiracel OD-H or Daicel Chiralpak AD columns.

\footnotetext{
${ }^{1}$ Still, W. C.; Kahn, M.; Mitra, A. J. Org. Chem. 1978, 43, 2923-2925.
} 


\section{Preparation of starting materials}

p-Toluenesulfonyl (Tosyl) aryl aldimines 2, 7a-k, 10a-c were prepared in moderate to good yields according to known procedures. ${ }^{2}$ 2-Methylenecyclopropane carboxylic acid diphenylamide $\mathbf{1}$ and 2methylenecyclopropanecarboxylic acid phenyl(pyridy-2-yl)amide $\mathbf{9}$ were prepared from 2-methylenecyclopropane carboxylic acid ethyl ester and 2-methylenecyclopropane carboxylic acid. ${ }^{3}$ The bis(oxazoline)-type ligands (S)-4, (S)-5, $(R, S)-\mathbf{6 a - c}$ and $(S, R)-\mathbf{6 c}$ were synthesized according to literature procedures. ${ }^{4}$

\section{2-Methylenecyclopropane carboxylic acid diphenylamide $1^{5}$}

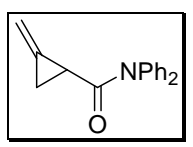

To a solution of MCP carboxylic acid $(3.96 \mathrm{~g}, 40 \mathrm{mmol})$ and triethylamine $(5.58 \mathrm{~mL}, 40 \mathrm{mmol})$ in THF $(150 \mathrm{~mL})$ was added isobutylchloroformate $(5.19 \mathrm{~mL}, 40 \mathrm{mmol})$ at $0{ }^{\circ} \mathrm{C}$. The solution was stirred at $0{ }^{\circ} \mathrm{C}$ for $15 \mathrm{~min}$ followed by the addition of lithium diphenylamide which was prepared by adding $n$-BuLi (17.6 mL, $44 \mathrm{mmol}, 2.5 \mathrm{M}$ solution in hexanes) into a solution of diphenylamine $(7.45 \mathrm{~g}, 44 \mathrm{mmol})$ in $\mathrm{THF}(20 \mathrm{~mL})$ at $-78{ }^{\circ} \mathrm{C}$ and warming to room temperature for $30 \mathrm{~min}$. The resulting mixture was stirred at room temperature for 4 hours. After being quenched with saturated $\mathrm{NH}_{4} \mathrm{Cl}$ solution, the reaction mixture was extracted with ethyl acetate $(2 \times 250 \mathrm{~mL})$. The combined organic extracts were washed with $\mathrm{H}_{2} \mathrm{O}(250 \mathrm{~mL})$, brine $(250 \mathrm{~mL})$ and dried over $\mathrm{MgSO}_{4}$, filtered and concentrated. Flash chromatography on silica gel (Hexanes:Ethyl Acetate 8:1) furnished the product $1(6.5 \mathrm{~g}, 26 \mathrm{mmol}, 65 \%)$ as a beige solid. $\mathrm{mp}=52{ }^{\circ} \mathrm{C}, R f=0.45$ (Hexanes:EtOAc 3:1); IR $\left(\mathrm{CH}_{2} \mathrm{Cl}_{2}\right)$ v (cm-1) 3062, 3036, 2991, 1742, 1668, 1594, 1490, 1448, 1361, 1251, 1177, 1158, 1103, 1074, 1025, 1003, 971, 900, 754, 755, 700, 616; ${ }^{1} \mathrm{H}-\mathrm{NMR}\left(300 \mathrm{MHz}, \mathrm{CDCl}_{3}\right) \delta 7.46-7.14(\mathrm{~m}, 10 \mathrm{H})$, 5.51-5.44 (m, 2H), 2.24-2.15 (m, 1H), 2.05-1.96 (m, 1H), 1.53-1.43 (m, 1H); ${ }^{13} \mathrm{C}-\mathrm{NMR}(75 \mathrm{MHz}$, $\left.\mathrm{CDCl}_{3}\right) \delta 170.7,142.9,132.4,129.4,128.5,127.9,127.1,103.4,19.9,11.5$; HRMS Calcd for $\mathrm{C}_{17} \mathrm{H}_{15} \mathrm{NO}(\mathrm{M})^{+}: 249.1154$ Found: 249.1166 .

\section{2-Methylenecyclopropanecarboxylic acid phenyl(pyridy-2-yl)amide 9}

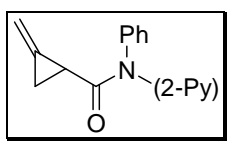

To a solution of MCP carboxylic acid (3.96 g, $40 \mathrm{mmol}, 1$ equiv) and triethylamine (5.58 $\mathrm{mL}, 40$ mmol, 1 equiv) in THF $(150 \mathrm{~mL})$ was added isobutylchloroformate $(5.19 \mathrm{~mL}, 40 \mathrm{mmol}, 1$ equiv) at $0{ }^{\circ} \mathrm{C}$. The solution was stirred at $0{ }^{\circ} \mathrm{C}$ for 15 min followed by the addition of lithium $\mathrm{N}$-phenyl(2pyridyl)amide which was prepared by adding $n$-BuLi $(17.6 \mathrm{~mL}, 2.5 \mathrm{M}$ solution in hexanes, 44 mmol, 1.1 equiv) into a solution of $N$-phenyl(2-pyridyl)amine (7.45 g, $44 \mathrm{mmol}, 1.1$ equiv) in THF $(20 \mathrm{~mL})$ at $-78{ }^{\circ} \mathrm{C}$ and warming to room temperature for $30 \mathrm{~min}$. The resulting mixture was stirred at room temperature for 6 hours. After being quenched with saturated $\mathrm{NH}_{4} \mathrm{Cl}$ solution, the reaction mixture was extracted with ethyl acetate $(2 \times 250 \mathrm{~mL})$. The combined organic extracts were washed

\footnotetext{
${ }^{2}$ (a) Jennings, W. B.; Lovely, C. J. Tetrahedron 1991, 47, 5561-5568. (b) Lee, K. Y.; Lee, C. G.; Kim, J. N. Tetrahedron Lett. 2003, 44, 1231-1234.

${ }^{3}$ Lai, M.; Liu, L.; Liu, H.-W. J. Am. Chem. Soc. 1991, 113, 7388-7397.

${ }^{4}$ (a) For the synthesis of 4, see: Evans, D. A.; Johnson, J. S. J. Am. Chem. Soc. 1998, 120, 4895-4896. (b) For the synthesis of 5, see: Nishiyama, H.; Kondo, M.; Nakamura, T.; Itoh, K. Organometallics 1991, 10, 500-508. (c) For the synthesis of $6 \mathbf{a}, \mathbf{6 b}$ and $\mathbf{6 c}$, see: Sibi, M. P.; Ji, J. J. Org. Chem. 1997, 62, 3800-3801 and Barnes, D. M.; Ji, J.; Fickes, M. A.; Fitzgerald, M. A.; King, S. A.; Morton, H. E.; Plagge, F. A.; Preskill, M.; Wagaw, S. H.; Wittenberger, S. J.; Zhang, J. J. Am. Chem. Soc. 2002, 124, 13097-13105.

${ }^{5}$ Lautens, M.; Han, W. J. Am. Chem. Soc. 2002, 124, 6312-6316.
} 
with $\mathrm{H}_{2} \mathrm{O}(250 \mathrm{~mL})$, brine $(250 \mathrm{~mL})$ and dried over $\mathrm{MgSO}_{4}$, filtered and concentrated. Flash chromatography on silica gel (Hexanes:Ethyl Acetate 8:1) furnished the product $9(6.3 \mathrm{~g}, 25 \mathrm{mmol}$, $63 \%$ ) as a pale yellow solid. $\mathrm{mp}=62{ }^{\circ} \mathrm{C} ; R f=0.52$ (Hexanes:EtOAc 3:1); IR $\left(\mathrm{CH}_{2} \mathrm{Cl}_{2}\right)$ v 3032, 3011, 2989, 1673, 1585, 1491, 1466, 1432, 1356, 1239, 1185, 1105, 904, 778, $699 \mathrm{~cm}^{-1}$; ${ }^{1} \mathrm{H}-\mathrm{NMR}$ $\left(400 \mathrm{MHz}, \mathrm{CDCl}_{3}\right) \delta 8.42(\mathrm{dm}, 1 \mathrm{H}, J=4.0 \mathrm{~Hz}), 7.73(\mathrm{tm}, 1 \mathrm{H}, J=7.2 \mathrm{~Hz}), 7.56(\mathrm{~d}, 1 \mathrm{H}, J=8.0 \mathrm{~Hz})$, 7.48-7.30 (m, 5H), $7.11(\mathrm{~m}, 1 \mathrm{H}), 5.03(\mathrm{~m}, 1 \mathrm{H}), 5.47(\mathrm{~m}, 1 \mathrm{H}), 2.22-2.17(\mathrm{~m}, 1 \mathrm{H}), 2.05-1.97(\mathrm{~m}, 1 \mathrm{H})$, 1.54-1.45 (m, 1H); ${ }^{13} \mathrm{C}-\mathrm{NMR}\left(100 \mathrm{MHz}, \mathrm{CDCl}_{3}\right) \delta 171.6,155.3,149.0,141.8,137.9,132.3,129.7$, 129.0, 127.8, 121.4, 103.7, 20.2, 11.6; HRMS Calcd for $\mathrm{C}_{16} \mathrm{H}_{14} \mathrm{~N}_{2} \mathrm{O}(\mathrm{M})^{+}: 249.1028$ Found: 249.1022 . 


\section{Enantioselective Ring Expansion of Methylenecyclopropyl Amides}

\section{General Procedure:}

To a flame dried flask were added $\mathrm{MgI}$ (17 mg, $0.06 \mathrm{mmol}, 30 \mathrm{~mol} \%)$ and CP-Indabox $6 \mathrm{c}$ (32 mg, $0.09 \mathrm{mmol}, 45 \mathrm{~mol} \%)$. THF ( $1 \mathrm{~mL})$ was added and the mixture was stirred at room temperature. After 2 hours, a solution of MCP $1(0.20 \mathrm{mmol}, 50 \mathrm{mg}, 1$ equiv. $)$ and $N$-tosyl aldimine $(0.24 \mathrm{mmol}$, 1.2 equiv.) in THF (1.5 mL) was added dropwise. The resulting yellow suspension was stirred for 1 hour at room temperature before heating to reflux. Reaction completion was monitored by TLC. The reaction was then cooled to room temperature, diluted with ethyl acetate $(2.0 \mathrm{~mL})$, and quenched with saturated aqueous sodium sulfite $(2.0 \mathrm{~mL})$. The aqueous phase was then extracted with ethyl acetate $(3 \times 15 \mathrm{~mL})$ and the combined organic layers were washed with $\mathrm{H}_{2} \mathrm{O}$ and brine, dried over $\mathrm{Na}_{2} \mathrm{SO}_{4}$, filtered and concentrated in vacuo.

\section{(+)-(2R,3R)-4-Methylene-1-(toluene-4-sulfonyl)-2-(4-trifluoromethyl-phenyl)-} pyrrolidine-3-carboxylic acid diphenylamide 3

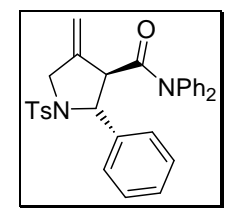

Following the general procedure outlined above (refluxed for 12 hours) for the enantioselective ring expansion of 2-methylenecyclopropane carboxylic acid diphenylamide $\mathbf{1}$ in the presence of aldimine 2 using ligand $(R, S)$-CP-Indabox 6c. After workup, purification by chromatography on silica gel (Gradient Hexanes:EtOAc; 5:1 to 3:1) gave methylene pyrrolidine $\mathbf{3}$ as a white solid (61 $\mathrm{mg}, 60 \%, 84 \%$ ee). $\mathrm{mp} 76-78{ }^{\circ} \mathrm{C}, R f=0.26$ (Hexanes:EtOAc 3:1); $[\alpha]_{\mathrm{D}}+66.4\left(c 1.0, \mathrm{CHCl}_{3}\right)$; IR $\left(\mathrm{CH}_{2} \mathrm{Cl}_{2}\right) \vee 3060,3035,2933,1674,1592,1490,1445,1349,1292,1164,1094,1030,909,836$, 753, $696 \mathrm{~cm}^{-1}$; ${ }^{1} \mathrm{H}-\mathrm{NMR}\left(300 \mathrm{MHz}, \mathrm{CDCl}_{3}\right) \delta 7.60(\mathrm{~d}, 2 \mathrm{H}, J=8.1 \mathrm{~Hz}), 7.37-6.97(\mathrm{~m}, 15 \mathrm{H}), 6.62$ (bs, 2H), $5.05(\mathrm{~m}, 1 \mathrm{H}), 4.99(\mathrm{~m}, 1 \mathrm{H}), 4.71(\mathrm{~d}, 1 \mathrm{H}, J=7.8 \mathrm{~Hz}), 4.26(\mathrm{bd}, 1 \mathrm{H}, J=13.9 \mathrm{~Hz}), 4.04$ $(\mathrm{dm}, 1 \mathrm{H}, J=13.9 \mathrm{~Hz}), 3.72(\mathrm{bd}, 1 \mathrm{H}, J=7.8 \mathrm{~Hz}), 2.39(\mathrm{~s}, 3 \mathrm{H}) ;{ }^{13} \mathrm{C}-\mathrm{NMR}\left(75 \mathrm{MHz}, \mathrm{CDCl}_{3}\right) \delta$ 169.7, 143.8, 142.3, 139.9, 132.6, 129.6, 128.5, 128.1, 128.0, 126.9, 126.4, 108.5, 68.2, 57.9, 54.4, 21.6; HRMS Calcd for $\mathrm{C}_{31} \mathrm{H}_{28} \mathrm{~N}_{2} \mathrm{O}_{3} \mathrm{~S}(\mathrm{M})^{+}$: 508.1664 Found: 508.1672. Chiral HPLC: Chiralcel OD-H column, 94:06 Hexanes: $i-\mathrm{PrOH}, 1 \mathrm{~mL} / \mathrm{min}, 30{ }^{\circ} \mathrm{C}, \mathrm{t}_{1}=46.0 \mathrm{~min}, \mathrm{t}_{2}=54.8 \mathrm{~min}$.

\section{(+)-(2R,3R)-4-Methylene-1-(toluene-4-sulfonyl)-2-(2-trifluoromethyl-phenyl)- pyrrolidine-3-carboxylic acid diphenylamide 8a}

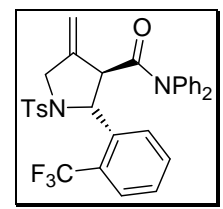

Following the general procedure outlined above (refluxed for 12 hours) for the enantioselective ring expansion of 2-methylenecyclopropane carboxylic acid diphenylamide $\mathbf{1}$ in the presence of aldimine 7a using ligand $(R, S)$-CP-Indabox 6c. After workup, purification by chromatography on silica gel (Gradient Hexanes:EtOAc; 5:1 to 3:1) gave methylene pyrrolidine 8a as a pale yellow solid (74 mg, 64\%, 86\% ee). mp 215-217 ${ }^{\circ} \mathrm{C}, R f=0.27$ (Hexanes:EtOAc 3:1); $[\alpha]_{\mathrm{D}}+92.8$ (c 2.0, $\left.\mathrm{CHCl}_{3}\right)$; IR $\left(\mathrm{CH}_{2} \mathrm{Cl}_{2}\right) \vee 3063,3038,2918,2842,1667,1617,1594,1490,1453,1348,1311,1286$, 1156, 1113, 1112, 1056, 1034, 1002, 914, 839, 810, 751, 700, $656 \mathrm{~cm}^{-1}$; ${ }^{1} \mathrm{H}-\mathrm{NMR}(300 \mathrm{MHz}$, $\left.\mathrm{CDCl}_{3}\right) \delta$ 7.82-7.74 (m, 2H), 7.66-7.42 (m, 3H), 7.38-7.08 (m, 9H), 7.04-6.94 (m, 4H), 5.53 (s, 1H), $5.19(\mathrm{~s}, 1 \mathrm{H}), 4.68(\mathrm{~m}, 1 \mathrm{H}), 4.28(\mathrm{~d}, 1 \mathrm{H}, J=11.9 \mathrm{~Hz}), 4.12(\mathrm{dd}, 1 \mathrm{H}, J=12.1,1.1 \mathrm{~Hz}), 3.45(\mathrm{~s}, 1 \mathrm{H})$, $2.40(\mathrm{~s}, 3 \mathrm{H}) ;{ }^{13} \mathrm{C}-\mathrm{NMR}\left(75 \mathrm{MHz}, \mathrm{CDCl}_{3}\right) \delta 170.0,143.8,142.2,141.5,133.8,132.5,129.6,129.1$, 
128.9, 128.5, 128.4, 127.6, 126.4, 126.0, 125.8, 125.7, 122.9, 112.3, 62.9, 57.4, 53.9, 21.7; HRMS Calcd for $\mathrm{C}_{32} \mathrm{H}_{27} \mathrm{~F}_{3} \mathrm{~N}_{2} \mathrm{O}_{3} \mathrm{~S}(\mathrm{M})^{+}:$576.1695 Found: 576.1681. Chiral HPLC: Chiralcel OD-H column, 94:06 Hexanes: $i-\mathrm{PrOH}, 1 \mathrm{~mL} / \mathrm{min}, 30^{\circ} \mathrm{C}, \mathrm{t}_{1}=15.9 \mathrm{~min}, \mathrm{t}_{2}=24.3 \mathrm{~min}$.

\section{(-)-(2S,3S)-4-Methylene-1-(toluene-4-sulfonyl)-2-(2-trifluoromethyl-phenyl)- pyrrolidine-3-carboxylic acid diphenylamide ent-8a}

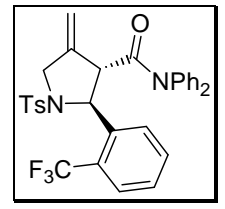

Following the general procedure outlined above (refluxed for 12 hours) for the enantioselective ring expansion of 2-methylenecyclopropane carboxylic acid diphenylamide $\mathbf{1}$ in the presence of aldimine 7a using ligand $(S, R)$-CP-Indabox 6c. After workup, purification by chromatography on silica gel (Gradient Hexanes:EtOAc; 5:1 to 3:1) gave methylene pyrrolidine ent-8a as a colourless oil (70 mg, 61\%, 82\% ee). $R f=0.26$ (Hexanes:EtOAc 3:1); $[\alpha]_{\mathrm{D}}-88.2\left(\right.$ c 2.3, $\left.\mathrm{CHCl}_{3}\right) ; \mathrm{IR}\left(\mathrm{CH}_{2} \mathrm{Cl}_{2}\right)$ $v$ 3063, 3038, 2918, 2842, 1667, 1617, 1594, 1490, 1453, 1348, 1311, 1286, 1156, 1113, 1112, 1056, 1034, 1002, 914, 839, 810, 751, 700, $656 \mathrm{~cm}^{-1}$; ${ }^{1} \mathrm{H}-\mathrm{NMR}\left(300 \mathrm{MHz}, \mathrm{CDCl}_{3}\right) \delta$ 7.82-7.74 (m, 2H), 7.66-7.42 (m, 3H), 7.38-7.08 (m, 9H), 7.04-6.94 (m, 4H), $5.53(\mathrm{~s}, 1 \mathrm{H}), 5.19(\mathrm{~s}, 1 \mathrm{H}), 4.68(\mathrm{~m}$, $1 \mathrm{H}), 4.28(\mathrm{~d}, 1 \mathrm{H}, J=11.9 \mathrm{~Hz}), 4.12(\mathrm{dd}, 1 \mathrm{H}, J=12.1,1.1 \mathrm{~Hz}), 3.45(\mathrm{~s}, 1 \mathrm{H}), 2.40(\mathrm{~s}, 3 \mathrm{H}) ;{ }^{13} \mathrm{C}-$ NMR $\left(75 \mathrm{MHz}, \mathrm{CDCl}_{3}\right) \delta 170.0,143.8,142.2,141.5,133.8,132.5,129.6,129.1,128.9,128.5$, $128.4,127.6,126.4,126.0,125.8,125.7,122.9,112.3,62.9,57.4,53.9,21.7$. Chiral HPLC: Chiralcel OD-H column, 94:06 Hexanes: $i-\mathrm{PrOH}, 1 \mathrm{~mL} / \mathrm{min}, 30^{\circ} \mathrm{C}, \mathrm{t}_{1}=15.9 \mathrm{~min}, \mathrm{t}_{2}=24.3 \mathrm{~min}$.

(+)-(2R,3R)-4-Methylene-1-(toluene-4-sulfonyl)-2-(4-trifluoromethyl-phenyl)pyrrolidine-3-carboxylic acid diphenylamide $8 \mathrm{~b}$

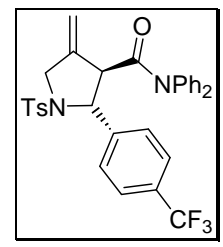

Following the general procedure outlined above (refluxed for 12 hours) for the enantioselective ring expansion of 2-methylenecyclopropane carboxylic acid diphenylamide $\mathbf{1}$ in the presence of aldimine $\mathbf{7 b}$ using ligand $(R, S)$-CP-Indabox 6c. After workup, purification by chromatography on silica gel (Gradient Hexanes:EtOAc; 5:1 to 3:1) gave methylene pyrrolidine $\mathbf{8 b}$ as a pale yellow solid $\left(60 \mathrm{mg}, 52 \%, 82 \%\right.$ ee). $\mathrm{mp} 180-182{ }^{\circ} \mathrm{C}$ (ethyl acetate), $R f=0.27$ (Hexanes:EtOAc $3: 1$ ); $[\alpha]_{\mathrm{D}}+62.4\left(c 1.0, \mathrm{CHCl}_{3}\right) ;$ IR $\left(\mathrm{CH}_{2} \mathrm{Cl}_{2}\right) \vee 3063,2924,2862,1671,1617,1595,1489,1451,1423$, 1348, 1323, 1160, 1119, 1066, 1013, 903, 850, 812, 753, 737, 699, $661 \mathrm{~cm}^{-1} ;{ }^{1} \mathrm{H}-\mathrm{NMR}(300 \mathrm{MHz}$, $\left.\mathrm{CDCl}_{3}\right) \delta$ 7.62-7.58 (m, 4H), 7.38-7.10 (m, 10H), 7.07-6.98 (m, 2H), $6.63(\mathrm{bs}, 2 \mathrm{H}), 5.09(\mathrm{~m}, 1 \mathrm{H})$, $5.01(\mathrm{~m}, 1 \mathrm{H}), 4.80(\mathrm{~d}, 1 \mathrm{H}, J=7.5 \mathrm{~Hz}), 4.28(\mathrm{~d}, 1 \mathrm{H}, J=13.6 \mathrm{~Hz}), 4.07(\mathrm{dm}, 1 \mathrm{H}, J=13.7 \mathrm{~Hz}), 3.68$ $(\mathrm{dm}, 1 \mathrm{H}, J=7.3 \mathrm{~Hz}), 2.42$ (s, 3H); ${ }^{13} \mathrm{C}-\mathrm{NMR}\left(75 \mathrm{MHz}, \mathrm{CDCl}_{3}\right) \delta 169.5,144.5,144.4,142.1,132.7$, 130.1, 129.9, 129.1, 128.3, 127.4, 126.8, 126.2, 125.7, 109.3, 67.6, 58.0, 54.5, 21.8; HRMS Calcd for $\mathrm{C}_{32} \mathrm{H}_{27} \mathrm{~F}_{3} \mathrm{~N}_{2} \mathrm{O}_{3} \mathrm{~S}(\mathrm{M})^{+}$: 576.1695 Found: 576.1702. Chiral HPLC: Chiralpak AD column, 95:05 Hexanes: $i-\mathrm{PrOH}, 1 \mathrm{~mL} / \mathrm{min}, 30^{\circ} \mathrm{C}, \mathrm{t}_{1}=36.2 \mathrm{~min}, \mathrm{t}_{2}=51.9 \mathrm{~min}$. 
(+)-(2R,3R)-2-(2-Bromo-phenyl)-4-methylene-1-(toluene-4-sulfonyl)pyrrolidine-3-carboxylic acid diphenylamide 8c

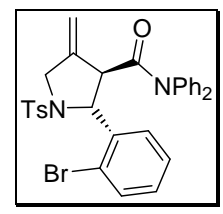

Following the general procedure outlined above (refluxed for 12 hours) for the enantioselective ring expansion of 2-methylenecyclopropane carboxylic acid diphenylamide $\mathbf{1}$ in the presence of aldimine 7c using ligand $(R, S)$-CP-Indabox 6c. After workup, purification by chromatography on silica gel (Gradient Hexanes:EtOAc; 5:1 to 3:1) gave methylene pyrrolidine 8c as a white solid (61 mg, 52\%, 74\% ee). mp $182.5^{\circ} \mathrm{C}, R f=0.27$ (Hexanes:EtOAc 3:1); $[\alpha]_{\mathrm{D}}+52.4$ (c 1.0, $\mathrm{CHCl}_{3}$ ); IR $\left(\mathrm{CH}_{2} \mathrm{Cl}_{2}\right)$ v 3061, 2984, 2923, 2854, 1664, 1592, 1489, 1447, 1344, 1268, 1158, 1093, 1070, 1002, 906, 842, 807, 754, 735, 701, $659 \mathrm{~cm}^{-1}$; ${ }^{1} \mathrm{H}-\mathrm{NMR}\left(300 \mathrm{MHz}, \mathrm{CDCl}_{3}\right) \delta 7.79(\mathrm{~d}, 2 \mathrm{H}$, $J=8.1 \mathrm{~Hz}), 7.55(\mathrm{dd}, 1 \mathrm{H}, J=8.2,1.2 \mathrm{~Hz}), 7.41(\mathrm{dd}, 1 \mathrm{H}, J=7.8,1.6 \mathrm{~Hz}), 7.36-7.09(\mathrm{~m}, 10 \mathrm{H})$, 7.08-6.91 (m, 4H), $5.45(\mathrm{bs}, 1 \mathrm{H}), 5.09(\mathrm{~d}, 1 \mathrm{H}, J=1.5 \mathrm{~Hz}),, 4.73(\mathrm{~s}, 1 \mathrm{H}), 4.26(\mathrm{dm}, 1 \mathrm{H}$, $J=12.6 \mathrm{~Hz}), 4.07$ (ddd, $1 \mathrm{H}, J=12.6,2.2,1.6 \mathrm{~Hz}), 3.59$ (bs, 1H), $2.41(\mathrm{~s}, 3 \mathrm{H}) ;{ }^{13} \mathrm{C}-\mathrm{NMR}(75 \mathrm{MHz}$, $\left.\mathrm{CDCl}_{3}\right) \delta 170.1,143.9,133.2,132.8,129.7,129.2,128.9,128.6,128.2,127.9,126.3,122.0,110.9$, 66.7, 56.6, 54.2, 21.8; HRMS Calcd for $\mathrm{C}_{31} \mathrm{H}_{28} \mathrm{BrN}_{2} \mathrm{O}_{3} \mathrm{~S}(\mathrm{M}+\mathrm{H})^{+}$: 587.1004 Found: 587.1000. Chiral HPLC: Chiralpak AD column, 95:05 Hexanes: $i-\mathrm{PrOH}, 1 \mathrm{~mL} / \mathrm{min}, 30{ }^{\circ} \mathrm{C}, \mathrm{t}_{1}=34.0 \mathrm{~min}$, $\mathrm{t}_{2}=39.1 \mathrm{~min}$.

(+)-(2R,3R)-2-(3-Bromo-phenyl)-4-methylene-1-(toluene-4-sulfonyl)pyrrolidine-3-carboxylic acid diphenylamide 8d

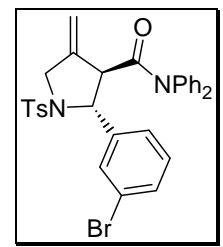

Following the general procedure outlined above (refluxed for 12 hours) for the enantioselective ring expansion of 2-methylenecyclopropane carboxylic acid diphenylamide $\mathbf{1}$ in the presence of aldimine 7d using ligand $(R, S)$-CP-Indabox 6c. After workup, purification by chromatography on silica gel (Hexanes:EtOAc; 4:1) gave methylene pyrrolidine 8d as a colourless oil $(63 \mathrm{mg}$, 54\%, $76 \%$ ee). $\mathrm{R}_{f}=0.23$ (Hexanes:EtOAc; 3:1); $[\alpha]_{\mathrm{D}}+47.0\left(\right.$ c 1.0, $\left.\mathrm{CHCl}_{3}\right)$; IR (Thin Film) v 3062, 1665, 1596, 1491, 1348, 1162, 1093, 704, 694, $662 \mathrm{~cm}^{-1}$; ${ }^{1} \mathrm{H}$ NMR $\left(300 \mathrm{MHz}, \mathrm{CDCl}_{3}\right) \delta$ 7.62-7.53 (m, 2H), 7.50-6.94 (m, 14H), $6.70(\mathrm{bs}, 2 \mathrm{H}), 5.07(\mathrm{~m}, 1 \mathrm{H}), 5.01(\mathrm{~m}, 1 \mathrm{H}), 4.73(\mathrm{~d}, 1 \mathrm{H}, J=7.6 \mathrm{~Hz}), 4.24$ $(\mathrm{bd}, 1 \mathrm{H}, J=13.2 \mathrm{~Hz}), 4.09(\mathrm{~m}, 1 \mathrm{H}), 3.66(\mathrm{~m}, 1 \mathrm{H}), 2.40(\mathrm{~s}, 3 \mathrm{H}) ;{ }^{13} \mathrm{C}$ NMR $\left(75 \mathrm{MHz}, \mathrm{CDCl}_{3}\right)$ $\delta 169.6,144.3,142.4,142.2,133.2,131.3,130.2,130.1,130.0,129.9,128.2,126.0,122.9,67.6$, 58.0, 54.4, 21.8; HRMS calcd for $\mathrm{C}_{31} \mathrm{H}_{27} \mathrm{BrN}_{2} \mathrm{O}_{3} \mathrm{~S}\left(\mathrm{M}^{+}\right)$586.0926, found 586.0926. Chiral HPLC: Chiralcel OD-H column, 90:10 Hexanes: $i-\mathrm{PrOH}, 1 \mathrm{~mL} / \mathrm{min}, 30^{\circ} \mathrm{C}, \mathrm{t}_{1}=26.1 \mathrm{~min}, \mathrm{t}_{2}=38.3 \mathrm{~min}$.

(+)-(2R,3R)-2-(4-Bromo-phenyl)-4-methylene-1-(toluene-4-sulfonyl)pyrrolidine-3-carboxylic acid diphenylamide $8 \mathrm{e}$

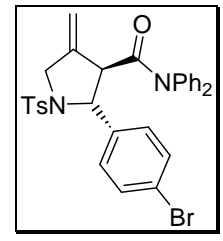

Following the general procedure outlined above (refluxed for 12 hours) for the enantioselective ring expansion of 2-methylenecyclopropane carboxylic acid diphenylamide $\mathbf{1}$ in the presence of 
aldimine 7e using ligand $(R, S)$-CP-Indabox 6c. After workup, purification by chromatography on silica gel (Gradient Hexanes:EtOAc; 5:1 to 3:1) gave methylene pyrrolidine 8e as a white solid (64 mg, 55\%, 78\% eе). mp 167-169 ${ }^{\circ} \mathrm{C}, R f=0.26$ (Hexanes:EtOAc:hexanes 3:1); $[\alpha]_{\mathrm{D}}+86.0(c 1.0$, $\left.\mathrm{CHCl}_{3}\right)$; IR $\left(\mathrm{CH}_{2} \mathrm{Cl}_{2}\right) \vee 3060,2927,2863,1671,1594,1487,1449,1348,1291,1161,1095,1073$, 1009, 902, 841, 813, 756, 731, 702, $661 \mathrm{~cm}^{-1}$; ${ }^{1} \mathrm{H}-\mathrm{NMR}\left(300 \mathrm{MHz}, \mathrm{CDCl}_{3}\right) \delta$ 7.64-7.44 (m, 4H), 7.39-6.99 (m, 12H), 6.79-6.64 (bs, 2H), $5.08(\mathrm{~s}, 1 \mathrm{H}), 5.02(\mathrm{~s}, 1 \mathrm{H}), 4.69(\mathrm{~d}, 1 \mathrm{H}, J=7.5 \mathrm{~Hz}), 4.26(\mathrm{~d}$, $1 \mathrm{H}, J=13.8 \mathrm{~Hz}), 4.05(\mathrm{dm}, 1 \mathrm{H}, J=13.8 \mathrm{~Hz}), 3.70(\mathrm{~d}, 1 \mathrm{H}, J=7.5 \mathrm{~Hz}), 2.44(\mathrm{~s}, 3 \mathrm{H}) ;{ }^{13} \mathrm{C}-\mathrm{NMR}$ $\left(75 \mathrm{MHz}, \mathrm{CDCl}_{3}\right) \delta 169.7,144.4,142.27,139.37,132.7,131.9,130.2,129.9,129.1,128.9,128.5$, 128.3, 126.2, 122.182, 109.1, 67.6, 57.9, 54.6, 21.8; HRMS Calcd for $\mathrm{C}_{31} \mathrm{H}_{27} \mathrm{BrN}_{2} \mathrm{O}_{3} \mathrm{~S}(\mathrm{M})^{+}$: 586.0926 Found: 586.0943. Chiral HPLC: Chiralpak AD column, 90:10 Hexanes:i-PrOH, $1 \mathrm{~mL} / \mathrm{min}, 30^{\circ} \mathrm{C}, \mathrm{t}_{1}=51.1 \mathrm{~min}, \mathrm{t}_{2}=64.7 \mathrm{~min}$.

(+)-(2R,3R)-2-(2,4-Dichloro-phenyl)-4-methylene-1-(toluene-4-sulfonyl)pyrrolidine-3-carboxylic acid diphenylamide $8 f$

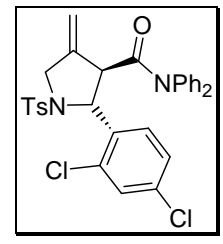

Following the general procedure outlined above (refluxed for 12 hours) for the enantioselective ring expansion of 2-methylenecyclopropane carboxylic acid diphenylamide $\mathbf{1}$ in the presence of aldimine 7f using ligand $(R, S)$-CP-Indabox 6c. After workup, purification by chromatography on silica gel (Gradient Hexanes:EtOAc; 5:1 to 3:1) gave methylene pyrrolidine 8f as a white solid (69 $\mathrm{mg}, 60 \%, 78 \%$ ee). $\mathrm{mp} 208{ }^{\circ} \mathrm{C}, R f=0.26$, (Hexanes:EtOAc 3:1); $[\alpha]_{\mathrm{D}}+102.8\left(c 1.0, \mathrm{CHCl}_{3}\right)$; IR $\left(\mathrm{CH}_{2} \mathrm{Cl}_{2}\right) \vee 3066,3038,2953,2925,2833,1673,1613,1596,1514,1494,1451,1346,1303,1247$, 1159, 1093, 1032, 906, 846, 814, 755, 727, 702, $657 \mathrm{~cm}^{-1} ;{ }^{1} \mathrm{H}-\mathrm{NMR}\left(300 \mathrm{MHz}, \mathrm{CDCl}_{3}\right) \delta 7.75(\mathrm{~d}$, $2 \mathrm{H}, J=8.3 \mathrm{~Hz}), 7.40-7.09(\mathrm{~m}, 11 \mathrm{H}), 7.09-6.91(\mathrm{bs}, 4 \mathrm{H}), 5.39(\mathrm{~d}, 1 \mathrm{H}, J=4.5 \mathrm{~Hz}), 5.1(\mathrm{~d}, 1 \mathrm{H}$, $J=1.5 \mathrm{~Hz}), 4.77(\mathrm{~m}, 1 \mathrm{H}), 4.24(\mathrm{dm}, 1 \mathrm{H}, J=12.7 \mathrm{~Hz}), 4.06(\mathrm{ddm}, 1 \mathrm{H}, J=12.7,1.6 \mathrm{~Hz}), 3.59(\mathrm{~m}$, 1H), $2.41(\mathrm{~s}, 3 \mathrm{H}) ;{ }^{13} \mathrm{C}-\mathrm{NMR}\left(75 \mathrm{MHz}, \mathrm{CDCl}_{3}\right) \delta 169.9,144.1,141.7,138.1,134.1,133.0,164$, 132.5, 130.0, 129.8, 129.3, 128.5, 127.7, 126.2, 111.0, 64.1, 56.3, 54.1, 21.8; HRMS Calcd for $\mathrm{C}_{31} \mathrm{H}_{26} \mathrm{Cl}_{2} \mathrm{~N}_{2} \mathrm{O}_{3} \mathrm{~S}(\mathrm{M})^{+}:$576.1041 Found: 576.1042. Chiral HPLC: Chiralpak AD column, 95:05 Hexanes: $i-\mathrm{PrOH}, 1 \mathrm{~mL} / \mathrm{min}, 30^{\circ} \mathrm{C}, \mathrm{t}_{1}=30.6 \mathrm{~min}, \mathrm{t}_{2}=45.1 \mathrm{~min}$.

\section{(+)-(2R,3R)-2-(2,4-Dimethyl-phenyl)-4-methylene-1-(toluene-4-sulfonyl)- pyrrolidine-3- carboxylic acid diphenylamide $8 \mathrm{~g}$}

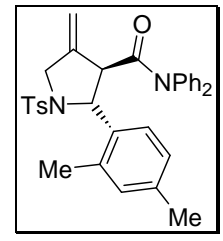

Following the general procedure outlined above (refluxed for 12 hours) for the enantioselective ring expansion of 2-methylenecyclopropane carboxylic acid diphenylamide $\mathbf{1}$ in the presence of aldimine $7 \mathbf{g}$ using ligand $(R, S)$-CP-Indabox 6c. After workup, purification by chromatography on silica gel (Gradient Hexanes:EtOAc; 5:1 to 3:1) gave methylene pyrrolidine $\mathbf{8 g}$ as a pale yellow solid (76 mg, 71\%, 71\% ee). mp 163-165 ${ }^{\circ} \mathrm{C}, R f=0.24$ (Hexanes:EtOAc 3:1); $[\alpha]_{\mathrm{D}}+87.0$ (c 1.0, $\left.\mathrm{CHCl}_{3}\right)$; IR $\left(\mathrm{CH}_{2} \mathrm{Cl}_{2}\right) \vee 3063,3038,2924,2861,1674,1596,1489,1451,1351,1291,1157,1072$, 1031, 1003, 902, 811, 758, 733, 702, $658 \mathrm{~cm}^{-1}$; ${ }^{1} \mathrm{H}-\mathrm{NMR}\left(300 \mathrm{MHz}, \mathrm{CDCl}_{3}\right) \delta 7.59(\mathrm{~d}, 2 \mathrm{H}$, $J=8.2 \mathrm{~Hz}), 7.34-6.87(\mathrm{~m}, 13 \mathrm{H}), 6.71-6.52(\mathrm{bs}, 2 \mathrm{H}), 5.09-4.94(\mathrm{~m}, 3 \mathrm{H}), 4.25(\mathrm{dm}, 1 \mathrm{H}$, $J=13.8 \mathrm{~Hz}), 4.06(\mathrm{ddm}, 1 \mathrm{H}, J=13.7,1.9 \mathrm{~Hz}), 3.76(\mathrm{~d}, 1 \mathrm{H}, J=7.5 \mathrm{~Hz}), 2.39(\mathrm{~s}, 3 \mathrm{H}), 2.34(\mathrm{~s}, 3 \mathrm{H})$, 
$2.29(\mathrm{~s}, 3 \mathrm{H}) ;{ }^{13} \mathrm{C}-\mathrm{NMR}\left(75 \mathrm{MHz}, \mathrm{CDCl}_{3}\right) \delta 170.3,143.8,142.8,137.4,135.5,134.6,133.5,131.7$, $129.9,129.6,129.0,128.3,128.2,127.0,126.2$, 108.8, 65.5, 56.5, 54.4, 21.7, 21.3, 19.6; HRMS Calcd for $\mathrm{C}_{33} \mathrm{H}_{33} \mathrm{~N}_{2} \mathrm{O}_{3} \mathrm{~S}(\mathrm{M}+\mathrm{H})^{+}:$537.2212 Found: 537.2196. Chiral HPLC: Chiralpak AD column, 90:10 Hexanes: $i-\mathrm{PrOH}, 1 \mathrm{~mL} / \mathrm{min}, 30^{\circ} \mathrm{C}, \mathrm{t}_{1}=28.1 \mathrm{~min}, \mathrm{t}_{2}=34.7 \mathrm{~min}$.

\section{(+)-(2R,3R)-2-(2-Methoxy-phenyl)-4-methylene-1-(toluene-4-sulfonyl)- pyrrolidine-3-carboxylic acid diphenylamide $8 \mathrm{~h}$}

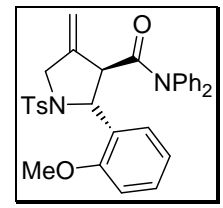

Following the general procedure outlined above (refluxed for 12 hours) for the enantioselective ring expansion of 2-methylenecyclopropane carboxylic acid diphenylamide $\mathbf{1}$ in the presence of aldimine $\mathbf{7 h}$ using ligand $(R, S)$-CP-Indabox 6c. After workup, purification by chromatography on silica gel (Hexanes:EtOAc; 3:1) gave methylene pyrrolidine $\mathbf{8 h}$ as a colourless oil (59 $\mathrm{mg}$, 55\%, 74\% ee). $\mathrm{R}_{f}=0.13$ (Hexanes:EtOAc; 3:1); $[\alpha]_{\mathrm{D}}+94.8\left(\right.$ c 1.0, $\left.\mathrm{CHCl}_{3}\right)$; IR (Thin Film) v 3062, 1668, 1597, 1491, 1343, 1160, 1097, 755, 703, $662 \mathrm{~cm}^{-1} ;{ }^{1} \mathrm{H}$ NMR $\left(300 \mathrm{MHz}, \mathrm{CDCl}_{3}\right) \delta$ 7.70-7.64 (m, 2H), 7.33-6.73 (m, 16H), $5.37(\mathrm{~d}, 1 \mathrm{H}, J=5.2 \mathrm{~Hz}), 5.04(\mathrm{~m}, 1 \mathrm{H}), 4.83(\mathrm{~m}, 1 \mathrm{H}), 4.23-4.09(\mathrm{~m}, 2 \mathrm{H})$, $3.77(\mathrm{~m}, 1 \mathrm{H}), 3.72(\mathrm{~s}, 3 \mathrm{H}), 2.38(\mathrm{~s}, 3 \mathrm{H}) ;{ }^{13} \mathrm{C} \mathrm{NMR}\left(100 \mathrm{MHz}, \mathrm{CDCl}_{3}\right) \delta 178.0,156.4,143.5,143.4$, 143.3, 134.7, 129.8, 129.5, 129.2, 129.1, 128.8, 128.3, 126.6, 120.9, 110.6, 109.7, 63.7, 55.4, 54.0, 21.8; HRMS calcd for $\mathrm{C}_{32} \mathrm{H}_{31} \mathrm{~N}_{2} \mathrm{O}_{4} \mathrm{~S}\left(\mathrm{M}+\mathrm{H}^{+}\right)$539.2004, found 539.2001. Chiral HPLC: Chiralcel OD-H column, 90:10 Hexanes: $i$ - $\mathrm{PrOH}, 1 \mathrm{~mL} / \mathrm{min}, 30{ }^{\circ} \mathrm{C}, \mathrm{t}_{1}=24.1 \mathrm{~min}, \mathrm{t}_{2}=29.7 \mathrm{~min}$.

\section{(+)-(2R,3R)-2-(4-Methoxy-phenyl)-4-methylene-1-(toluene-4-sulfonyl)-} pyrrolidine-3-carboxylic acid diphenylamide $8 \mathrm{i}$

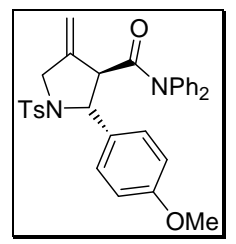

Following the general procedure outlined above (refluxed for 12 hours) for the enantioselective ring expansion of 2-methylenecyclopropane carboxylic acid diphenylamide $\mathbf{1}$ in the presence of aldimine 7i using ligand $(R, S)$-CP-Indabox 6c. After workup, purification by chromatography on silica gel (Gradient Hexanes:EtOAc; 5:1 to 3:1) gave methylene pyrrolidine 8i as a white solid (75 $\mathrm{mg}, 70 \%, 56 \%$ ee). $\mathrm{mp} 225-227{ }^{\circ} \mathrm{C} ; R f=0.21$ (Hexanes:EtOAc 3:1); $[\alpha]_{\mathrm{D}}+76.4\left(c\right.$ 1.0, $\left.\mathrm{CHCl}_{3}\right)$; IR $\left(\mathrm{CH}_{2} \mathrm{Cl}_{2}\right) \vee 3060,2918,2847,1674,1610,1594,1510,1490,1449,1345,1291,1246,1159,1094$, $1072,1027,843,808,752,698 \mathrm{~cm}^{-1}$; ${ }^{1} \mathrm{H}-\mathrm{NMR}\left(300 \mathrm{MHz}, \mathrm{CDCl}_{3}\right) \delta$ 7.61-7.56 (m, 2H), 7.32-7.05 $(\mathrm{m}, 10 \mathrm{H}), 7.09-6.96(\mathrm{~m}, 2 \mathrm{H}), 6.89-6.83(\mathrm{~m}, 2 \mathrm{H}), 6.74-6.61(\mathrm{bs}, 2 \mathrm{H}), 5.04(\mathrm{dm}, 1 \mathrm{H}, J=2.2 \mathrm{~Hz}), 4.99$ $(\mathrm{dm}, 1 \mathrm{H}, J=2.2 \mathrm{~Hz}), 4.62(\mathrm{~d}, 1 \mathrm{H}, J=7.9 \mathrm{~Hz}), 4.25(\mathrm{dm}, 1 \mathrm{H}, J=13.9 \mathrm{~Hz}), 4.00(\mathrm{ddd}, 1 \mathrm{H}, J=13.9$, 2.2, $2.2 \mathrm{~Hz}), 3.85(\mathrm{~s}, 3 \mathrm{H}), 3.72(\mathrm{~m}, 1 \mathrm{H}), 2.41(\mathrm{~s}, 3 \mathrm{H}) ;{ }^{13} \mathrm{C}-\mathrm{NMR}\left(75 \mathrm{MHz}, \mathrm{CDCl}_{3}\right) \delta 170.0,159.6$, 144.0, 142.6, 132.8, 131.8, 129.8, 129.1, 128.5, 128.5, 128.4, 126.6, 126.3, 114.0, 108.5, 68.0, 58.1, 55.6, 54.6, 21.8; HRMS Calcd for $\mathrm{C}_{32} \mathrm{H}_{30} \mathrm{~N}_{2} \mathrm{O}_{4} \mathrm{~S}(\mathrm{M})^{+}$: 538.1926 Found: 538.1914. Chiral HPLC: Chiralcel OD-H column, 90:10 Hexanes: $i-\mathrm{PrOH}, 1 \mathrm{~mL} / \mathrm{min}, 30^{\circ} \mathrm{C}, \mathrm{t}_{1}=35.3 \mathrm{~min}, \mathrm{t}_{2}=78.3 \mathrm{~min}$. 
(+)-(2R,3R)-2-Benzo[1,3] dioxol-5-yl-4-methylene-1-(toluene-4-sulfonyl)pyrrolidine-3-carboxylic acid diphenylamide $8 \mathbf{j}$

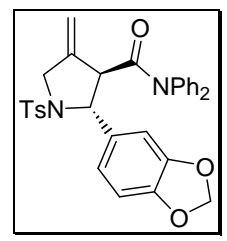

Following the general procedure outlined above (refluxed for 12 hours) for the enantioselective ring expansion of 2-methylenecyclopropane carboxylic acid diphenylamide $\mathbf{1}$ in the presence of aldimine 7j using ligand $(R, S)$-CP-Indabox 6c. After workup, purification by chromatography on silica gel (Gradient Hexanes:EtOAc; 5:1 to 3:1) gave methylene pyrrolidine $\mathbf{8 j}$ as a colourless oil (67 mg, 61\%, 64\% ee). $\mathrm{R}_{f}=0.11$ (Hexanes:EtOAc; 3:1); $[\alpha]_{\mathrm{D}}+73.2\left(c 1.0, \mathrm{CHCl}_{3}\right)$; IR (Thin Film) v 3062, 2921, 1672, 1490, 1346, 1248, 1163, 1094, 1038, 703, $663 \mathrm{~cm}^{-1} ;{ }^{1} \mathrm{H}$ NMR (400 MHz, $\left.\mathrm{CDCl}_{3}\right) \delta 7.78(\mathrm{~d}, 1 \mathrm{H}, J=8.3 \mathrm{~Hz}), 7.60(\mathrm{~d}, 2 \mathrm{H}, J=8.1 \mathrm{~Hz}), 7.34-6.97(\mathrm{~m}, 10 \mathrm{H}), 6.80-6.62(\mathrm{~m}, 4 \mathrm{H})$, $5.97(\mathrm{dd}, 2 \mathrm{H}, J=13.0,1.5 \mathrm{~Hz}), 5.05(\mathrm{~m}, 1 \mathrm{H}), 4.99(\mathrm{~m}, 1 \mathrm{H}), 4.63(\mathrm{~d}, 1 \mathrm{H}, J=7.6 \mathrm{~Hz}), 4.22(\mathrm{~d}, 1 \mathrm{H}$, $J=13.8 \mathrm{~Hz}), 4.02(\mathrm{dm}, 1 \mathrm{H}, J=13.8 \mathrm{~Hz}), 3.70(\mathrm{dm}, 1 \mathrm{H}, J=7.6 \mathrm{~Hz}), 2.40(\mathrm{~s}, 3 \mathrm{H}) ;{ }^{13} \mathrm{C}$ NMR $(100$ $\left.\mathrm{MHz}, \mathrm{CDCl}_{3}\right) \delta 170.0,148.0,147.6,144.0,142.4,133.9,132.9,129.8,129.0,128.6,128.3,126.6$, 120.7, 108.9, 108.1, 107.5, 101.3, 68.1, 57.9, 54.5, 21.7; HRMS calcd for $\mathrm{C}_{32} \mathrm{H}_{28} \mathrm{~N}_{2} \mathrm{O}_{5} \mathrm{~S}\left(\mathrm{M}^{+}\right)$ 552.1719, found 551.1719. Chiral HPLC: ChiralPak AD column, 85:15 Hexanes:i-PrOH, 1 $\mathrm{mL} / \mathrm{min}, 30{ }^{\circ} \mathrm{C}, \mathrm{t}_{1}=54.3 \mathrm{~min}, \mathrm{t}_{2}=60.8 \mathrm{~min}$.

(+)-Acetic acid (2R,3R)-4-[3-diphenylcarbamoyl-4-methylene-1-(toluene-4sulfonyl)-pyrrolidin-2-yl]-phenyl ester 8k

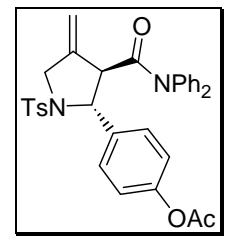

Following the general procedure outlined above (refluxed for 12 hours) for the enantioselective ring expansion of 2-methylenecyclopropane carboxylic acid diphenylamide $\mathbf{1}$ in the presence of aldimine $7 \mathbf{k}$ using ligand $(R, S)$-CP-Indabox 6c. After workup, purification by chromatography on silica gel (Hexanes:EtOAc; 3:1) gave methylene pyrrolidine 8k as a colourless oil $(67 \mathrm{mg}$, 59\%, $70 \%$ ee). $\mathrm{R}_{f}=0.08$ (Hexanes:EtOAc; 3:1); $[\alpha]_{\mathrm{D}}+104.7\left(\right.$ c 0.34, $\left.\mathrm{CHCl}_{3}\right)$; IR (Thin Film) v 3061, 1766, 1674, 1596, 1505, 1491, 1350, 1201, 1164, 1095, 735, 704, $664 \mathrm{~cm}^{-1} ;{ }^{1} \mathrm{H}$ NMR $(300 \mathrm{MHz}$, $\left.\mathrm{CDCl}_{3}\right) \delta$ 7.63-7.55 (m, 2H), 7.36-7.00 (m, 14H), 6.74-6.55 (m, 2H), $5.06(\mathrm{~m}, 1 \mathrm{H}), 5.00(\mathrm{~m}, 1 \mathrm{H})$, $4.71(\mathrm{~d}, 1 \mathrm{H}, J=7.9 \mathrm{~Hz}), 4.26(\mathrm{dm}, 1 \mathrm{H}, J=14.1 \mathrm{~Hz}), 4.03(\mathrm{~m}, 1 \mathrm{H}), 3.69(\mathrm{~m}, 1 \mathrm{H}), 2.39(\mathrm{~s}, 3 \mathrm{H}), 2.33$ $(\mathrm{s}, 3 \mathrm{H}) ;{ }^{13} \mathrm{C}$ NMR $\left(100 \mathrm{MHz}, \mathrm{CDCl}_{3}\right) \delta 170.0,169.7,150.7,144.2,142.3,137.7,143.1,132.5$, 130.3, 129.9, 129.1, 128.5, 128.3, 128.0, 126.7, 126.3, 122.0, 116.5, 108.8, 67.8, 58.2, 54.6, 21.8, 21.4; HRMS calcd for $\mathrm{C}_{33} \mathrm{H}_{31} \mathrm{~N}_{2} \mathrm{O}_{5} \mathrm{~S}\left(\mathrm{M}+\mathrm{H}^{+}\right)$567.1954, found 567.1956. Chiral HPLC: Chiralpak AD column, 93:07 Hexanes: $i-\mathrm{PrOH}, 1 \mathrm{~mL} / \mathrm{min}, 30{ }^{\circ} \mathrm{C}, \mathrm{t}_{1}=98.8 \mathrm{~min}, \mathrm{t}_{2}=105.3 \mathrm{~min}$.

(+)-(2R,3R)-2-Furan-2-yl-4-methylene-1-(toluene-4-sulfonyl)-pyrrolidine-3carboxylic acid diphenylamide 11a

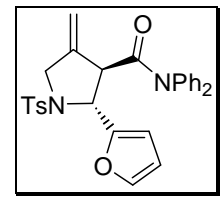

Following the general procedure outlined above (refluxed for 14 hours) for the enantioselective ring expansion of 2-methylenecyclopropane carboxylic acid diphenylamide $\mathbf{1}$ in the presence of aldimine 10a using ligand $(R, S)$-CP-Indabox 6c. After workup, purification by chromatography on 
silica gel (Gradient Hexanes:EtOAc; 4:1 to 2:1) gave methylene pyrrolidine 11a as a colourless oil (55 mg, 55\%, 71\% ee). $\mathrm{R}_{f}=0.17$ (Hexanes:EtOAc; 3:1); $[\alpha]_{\mathrm{D}}+98.8\left(\right.$ ( $\left.1.0 \mathrm{CHCl}_{3}\right)$; IR (Thin Film) v 3062, 2923, 1673, 1597, 1491, 1452, 1348, 1161, 1095, 736, 703, $660 \mathrm{~cm}^{-1} ;{ }^{1} \mathrm{H}$ NMR (400 MHz, $\left.\mathrm{CDCl}_{3}\right) \delta$ 7.65-7.60 (m, 2H), 7.37-7.01 (m, 11H), $6.89(\mathrm{bs}, 2 \mathrm{H}), 6.38-6.33(\mathrm{~m}, 2 \mathrm{H}), 5.06(\mathrm{~m}, 1 \mathrm{H})$, $5.03(\mathrm{~m}, 1 \mathrm{H}), 4.90(\mathrm{~d}, 1 \mathrm{H}, J=7.3 \mathrm{~Hz}), 4,17(\mathrm{dm}, 1 \mathrm{H}, J=13.4 \mathrm{~Hz}), 4.08-3.99(\mathrm{~m}, 2 \mathrm{H}), 2.38(\mathrm{~s}, 3 \mathrm{H})$; ${ }^{13} \mathrm{C}$ NMR $\left(100 \mathrm{MHz}, \mathrm{CDCl}_{3}\right) \delta 170.1,151.1,143.9,142.7,142.4,133.4,130.1,129.8,129.1,128.5$, 128.1, 126.3, 110.6, 109.2, 109.1, 61.1, 54.0, 53.8, 21.7; HRMS calcd for $\mathrm{C}_{29} \mathrm{H}_{27} \mathrm{~N}_{2} \mathrm{O}_{4} \mathrm{~S}(\mathrm{M}+\mathrm{H})^{+}$ 499.1686, found 499.1675. Chiral HPLC: Chiralcel OD-H column, 90:10 Hexanes:i-PrOH, $1 \mathrm{~mL} / \mathrm{min}, 30{ }^{\circ} \mathrm{C}, \mathrm{t}_{1}=27.1 \mathrm{~min}, \mathrm{t}_{2}=40.5 \mathrm{~min}$.

\section{(+)-(2R,3R)-2-Furan-3-yl-4-methylene-1-(toluene-4-sulfonyl)-pyrrolidine-3- carboxylic acid diphenylamide $11 b$}

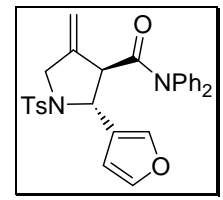

Following the general procedure outlined above (refluxed for 14 hours) for the enantioselective ring expansion of 2-methylenecyclopropane carboxylic acid diphenylamide $\mathbf{1}$ in the presence of aldimine 10b using ligand $(R, S)$-CP-Indabox 6c. After workup, purification by chromatography on silica gel (Hexanes:EtOAc; 4:1) gave methylene pyrrolidine $\mathbf{1 1 b}$ as a colourless oil $(66 \mathrm{mg}, 66 \%$, $72 \%$ ee). $\mathrm{R}_{f}=0.18$ (Hexanes:EtOAc; 3:1); $[\alpha]_{\mathrm{D}}+66.4\left(c\right.$ 1.0, $\left.\mathrm{CHCl}_{3}\right)$; IR (Thin Film) v 3062, 1674, 1596, 1491, 1347, 1161, 1095, 704, $664 \mathrm{~cm}^{-1} ;{ }^{1} \mathrm{H}$ NMR $\left(300 \mathrm{MHz}, \mathrm{CDCl}_{3}\right) \delta$ 7.69-7.61 (m, 2H), 7.41-6.91 (m, 14H), $6.08(\mathrm{~m}, 1 \mathrm{H}), 5.07(\mathrm{~m}, 1 \mathrm{H}), 5.02(\mathrm{~m}, 1 \mathrm{H}), 4.77(1 \mathrm{H}, \mathrm{d}, J=6.8 \mathrm{~Hz}), 4.20-4.00$ $(\mathrm{m}, 2 \mathrm{H}), 3.75(\mathrm{~m}, 1 \mathrm{H}), 2.39(\mathrm{~s}, 3 \mathrm{H}) ;{ }^{13} \mathrm{C}$ NMR $\left(75 \mathrm{MHz}, \mathrm{CDCl}_{3}\right) \delta 169.9,144,0,143.7,142.5$, $140.3,133.5,129.8,128.2,124.9,109.4,109.1,60.0,56.3,54.0,21.8$; HRMS calcd for $\mathrm{C}_{29} \mathrm{H}_{27} \mathrm{~N}_{2} \mathrm{O}_{4} \mathrm{~S}(\mathrm{M}+\mathrm{H})^{+}$499.1686, found 499.1693. Chiral HPLC: Chiralcel OD-H column, 90:10 Hexanes: $i-\mathrm{PrOH}, 1 \mathrm{~mL} / \mathrm{min}, 30^{\circ} \mathrm{C}, \mathrm{t}_{1}=28.1 \mathrm{~min}, \mathrm{t}_{2}=40,2 \mathrm{~min}$.

\section{(+)-(2R,3R)-1'-Methyl-4-methylene-1-(toluene-4-sulfonyl)-2,3,4,5-tetrahydro- 1H,1'H-[2,2']bipyrrolyl-3-carboxylic acid diphenylamide 11c}

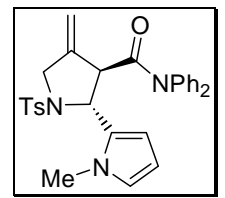

Following the general procedure outlined above (refluxed for 14 hours) for the enantioselective ring expansion of 2-methylenecyclopropane carboxylic acid diphenylamide $\mathbf{1}$ in the presence of aldimine 10c using ligand $(R, S)$-CP-Indabox 6c. After workup, purification by chromatography on silica gel (Gradient Hexanes:EtOAc; 4:1 to 2:1) gave methylene pyrrolidine 11c as an orange oil (68 mg, 67\%, 48\% ee). $\mathrm{R}_{f}=0.13$ (Hexanes:EtOAc; 3:1); $[\alpha]_{\mathrm{D}}+64.2\left(c\right.$ 1.0, $\mathrm{CHCl}_{3}$ ); IR (Thin Film) v 3061, 2924, 1676, 1597, 1491, 1352, 1294, 1165, 1093, 760, 704, $658 \mathrm{~cm}^{-1} ;{ }^{1} \mathrm{H}$ NMR (400 MHz, $\left.\mathrm{CDCl}_{3}\right) \delta$ 7.60-7.55 (m, 2H), 7.33-7.20 (m, 7H), 7.17-7.20 (m, 3H), 6.67-6.55 (m, 3H), 6.09 (dd, $1 \mathrm{H}, J=3.5,3.6 \mathrm{~Hz}), 6.02(\mathrm{dd}, 1 \mathrm{H}, J=3,5,2.0 \mathrm{~Hz}), 5,09(\mathrm{~m}, 1 \mathrm{H}), 5.06(\mathrm{~m}, 1 \mathrm{H}), 4.61(\mathrm{~d}, 1 \mathrm{H}, J=9.5$ $\mathrm{Hz}), 4.22(\mathrm{bd}, 1 \mathrm{H}, J=13.9 \mathrm{~Hz}), 3.94(\mathrm{dm}, 1 \mathrm{H}, J=9.5 \mathrm{~Hz}), 3.87(\mathrm{dm}, 1 \mathrm{H}, J=13.9 \mathrm{~Hz}), 3.35(\mathrm{~s}$, 3H), $2.39(\mathrm{~s}, 3 \mathrm{H}) ;{ }^{13} \mathrm{C}$ NMR $\left(100 \mathrm{MHz}, \mathrm{CDCl}_{3}\right) \delta 170.4,144.1,142.3,142.2,132.0,130.0,129.7$, 129.1, 128.6, 128.4, 126.6, 126.3, 124.6, 110.5, 108.7, 107.2, 62.6, 54.3, 54.2, 34.9, 21.7; HRMS calcd for $\mathrm{C}_{30} \mathrm{H}_{29} \mathrm{~N}_{3} \mathrm{O}_{3} \mathrm{~S}\left(\mathrm{M}^{+}\right)$511.1930, found 511.1938. Chiral HPLC: ChiralPak AD column, $85: 15$ hexane: $i-\mathrm{PrOH}, 1 \mathrm{~mL} / \mathrm{min}, 30{ }^{\circ} \mathrm{C}, \mathrm{t}_{1}=26.0 \mathrm{~min}, \mathrm{t}_{2}=28.9 \mathrm{~min}$. 


\section{(-)-(2R,3R)-4-Methylene-2-phenyl-1-(toluene-4-sulfonyl)-pyrrolidine-3- carboxylic acid phenyl-pyridin-2-yl-amide 11d}

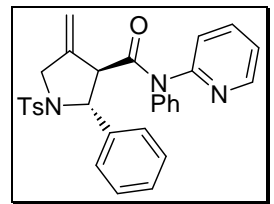

Following the general procedure outlined above (refluxed for 10 hours) for the enantioselective ring expansion of 2-methylenecyclopropane carboxylic acid phenyl(2-pyridyl)amide 9 in the presence of aldimine 2 using ligand $(R, S)$-CP-Indabox $\mathbf{6 c}$. After workup, purification by chromatography on silica gel (Gradient Hexanes:EtOAc; 4:1 to 2:1) gave methylene pyrrolidine 11d as a colourless oil (93 mg, 92\%, 78\% ee). $\mathrm{R}_{f}=0.14$ (Hexanes:EtOAc; 3:1); $[\alpha]_{\mathrm{D}}-71.0\left(c\right.$ 1.0, $\mathrm{CHCl}_{3}$ ); IR (Thin Film) v 3061, 1678, 1585, 1346, 1292, 1162, 1095, 757, 700, $662 \mathrm{~cm}^{-1} ;{ }^{1} \mathrm{H}$ NMR $\left(300 \mathrm{MHz}, \mathrm{CDCl}_{3}\right)$ $\delta 8.29(\mathrm{~m}, 1 \mathrm{H}), 7.65-7.54(\mathrm{~m}, 3 \mathrm{H}), 7.36-7.14(\mathrm{~m}, 10 \mathrm{H}), 7.12-7.00(\mathrm{~m}, 2 \mathrm{H}), 6.90-6.80(\mathrm{~m}, 2 \mathrm{H}), 5.08$ $(\mathrm{m}, 1 \mathrm{H}), 5.02(\mathrm{~m}, 1 \mathrm{H}), 4.87(\mathrm{~d}, 1 \mathrm{H}, J=6.5 \mathrm{~Hz}), 4.25(\mathrm{dm}, 1 \mathrm{H}, J=13.8 \mathrm{~Hz}), 4.11(\mathrm{~m}, 1 \mathrm{H}), 3.76(\mathrm{~m}$, $1 \mathrm{H}), 2.38(\mathrm{~s}, 3 \mathrm{H}) ;{ }^{13} \mathrm{C}$ NMR $\left(75 \mathrm{MHz}, \mathrm{CDCl}_{3}\right) \delta 170.8,154.8,149.1,143.9,142.5,141.1,140.6$, 138.1, 133.5, 129.8, 129.7, 128.7, 128.4, 128.3, 128.1, 128.0, 127.0, 122.0, 121.3, 109.7, 68.1, 58.5, 54.4, 21.8; HRMS calcd for $\mathrm{C}_{30} \mathrm{H}_{27} \mathrm{~N}_{3} \mathrm{O}_{3} \mathrm{~S}\left(\mathrm{M}^{+}\right)$509.1773, found 509.1772. Chiral HPLC: Chiralpak AD column, 80:20 Hexanes: $i-\mathrm{PrOH}, 1 \mathrm{~mL} / \mathrm{min}, 30^{\circ} \mathrm{C}, \mathrm{t}_{1}=28.4 \mathrm{~min}, \mathrm{t}_{2}=38.6 \mathrm{~min}$.

\section{Determination of the absolute configuration of methylenepyrrolidine 8i}

The absolute stereochemistry assignment was based upon the published ${ }^{6} \mathrm{X}$-Ray structure of the methylenepyrrolidine resulting from $\mathrm{MgI}_{2}$-mediated ring expansion of $\mathrm{MCP} \mathbf{1}$ with the chiral $p$-methoxy substituted aromatic sulfinime. After oxidation of its opposite enantiomer with $m$-CPBA, it was possible to assign the absolute configuration of the ring-expanded product $\mathbf{8 i}$ to be $(2 R, 3 R)$ by comparison of both optical rotations and chiral HPLC retention times.
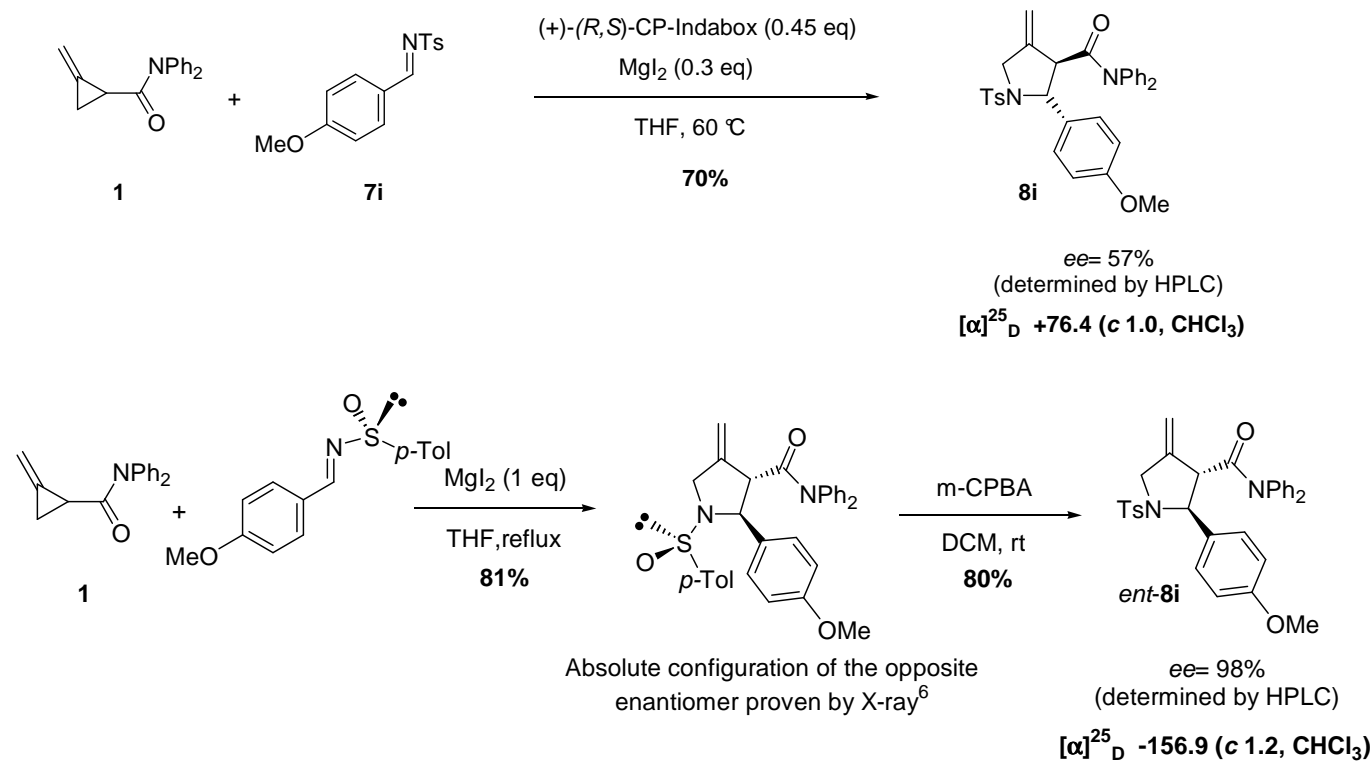
(-)-(2S,3S,SR)-2-(4-Methoxy-phenyl)-4-methylene-1-(toluene-4-sulfinyl)pyrrolidine-3-carboxylic acid diphenylamide $12^{6}$

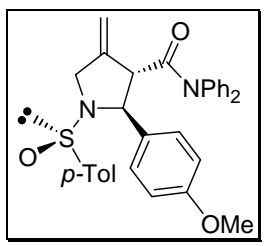

To a flame dried flask was added MCP (50 mg, $0.20 \mathrm{mmol}, 1$ equiv.), MgI2 (56 mg, $0.20 \mathrm{mmol}$, 1 equiv.) and sulfinimine ( $65 \mathrm{mg}, 0.24 \mathrm{mmol}, 1.2$ equiv.). THF was added ( $2.0 \mathrm{~mL}$ ) and the solution was stirred for 20 minutes at room temperature before heating to reflux. Reaction completion was monitored by TLC. The reaction was then cooled to room temperature, diluted with ethyl acetate $(2.0 \mathrm{~mL})$, and quenched with saturated aqueous sodium sulfite $(2.0 \mathrm{~mL})$. The aqueous layer was separated and $0.5 \mathrm{M}$ tetrasodium ethylenediaminetetraacetic acid salt (EDTA) was added (50 mL). The aqueous phase was then extracted with ethyl acetate $(3 \times 50 \mathrm{~mL})$ and the combined organic extracts were dried over $\mathrm{Na}_{2} \mathrm{SO}_{4}$, filtered and concentrated in vacuo. Purification by Flash chromatography on silica gel (Hexanes:EtOAc; 1:1) gave the ring expanded product $\mathbf{1 2}$ as a white solide (86 mg, 85\%). mp 175-177oC; $\mathrm{R}_{f}=0.39$ (Hexanes:EtOAc; 1:1); $[\alpha]_{\mathrm{D}}-53.4$ (c 1.0, $\mathrm{CHCl}$ ); IR $\left(\mathrm{CH}_{2} \mathrm{Cl}_{2}\right) 3004,2933,1675,1612,1594,1513,1491,1454,1364,1296,1250,1173,1156,1091$, 1068, 1031, 984, 884, 837, 812, 755, $703 \mathrm{~cm}^{-1}$; ${ }^{1} \mathrm{H}$ NMR (400 MHz, CDCl3) 7.47 (d, 2H, $J=8.4$ $\mathrm{Hz}), 7.34(\mathrm{~d}, 2 \mathrm{H}, J=8.4 \mathrm{~Hz}), 7.32-7.04(\mathrm{~m}, 10 \mathrm{H}), 6.97(\mathrm{~d}, 2 \mathrm{H}, J=8.8 \mathrm{~Hz}), 6.68(\mathrm{bs}, 2 \mathrm{H}), 5.13(\mathrm{~d}$, $1 \mathrm{H}, J=2.4 \mathrm{~Hz}), 5.05(\mathrm{~d}, 1 \mathrm{H}, J=10.0 \mathrm{~Hz}), 4.98(\mathrm{~d}, 1 \mathrm{H}, J=2.4 \mathrm{~Hz}), 4.15(\mathrm{dd}, 1 \mathrm{H}, J=14.4,2.4$ $\mathrm{Hz}), 3.89(\mathrm{~s}, 3 \mathrm{H}), 3.73(\mathrm{dm}, 1 \mathrm{H}, J=10.0 \mathrm{~Hz}), 3.06(\mathrm{~d}, 1 \mathrm{H}, J=14.4 \mathrm{~Hz}), 2.38(\mathrm{~s}, 3 \mathrm{H}) ;{ }^{13} \mathrm{C} \mathrm{NMR}$ (100 MHz, CDCl3) 170.1, 160.2, 144.5, 142.5, 142.4, 141.3, 140.9, 130.2, 129.9, 129.8, 129.7, $129.1,128.8,128.8,128.2,126.5,126.3,125.6,114.4,107.9,67.4,57.3,55.6,45.5,21.6$; HRMS calcd for $\mathrm{C}_{32} \mathrm{H}_{30} \mathrm{~N}_{2} \mathrm{O}_{3} \mathrm{~S}\left(\mathrm{M}^{+}\right)$523.2055, found: 523.2040.

\section{(-)-(2S,3S)-2-(4-Methoxy-phenyl)-4-methylene-1-(toluene-4-sulfonyl)- pyrrolidine-3-carboxylic acid diphenylamide ent-8i}

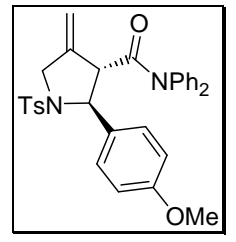

To a solution of ring-expanded product $12\left(52 \mathrm{mg}, 0.1 \mathrm{mmol}, 1\right.$ equiv.) in DCM (15 mL), at $0{ }^{\circ} \mathrm{C}$, was added m-CPBA ( $0.2 \mathrm{mmol}, 2$ equiv.) in one portion. After 1 hour at at $0{ }^{\circ} \mathrm{C}$, the reaction mixture was allowed to cool to room temperature and stirred for 12 hours. The reaction was then quenched with a saturated aqueous solution of $\mathrm{NaHCO}_{3}(15 \mathrm{~mL})$. The biphasic mixture was stirred for 1 hour. The organic layer was next washed with $\mathrm{H}_{2} \mathrm{O}$ and brine, dried over $\mathrm{Na}_{2} \mathrm{SO}_{4}$, filtered and concentrated in vacuo. Purification by chromatography on silica gel (Gradient Hexanes:EtOAc; 5:1 to 3:1) then gave methylene pyrrolidine ent-8i as a white solid (86 $\mathrm{mg}, 80 \%, 98 \%$ ee $)$. mp 223-225 ${ }^{\circ} \mathrm{C} ; R f=0.21$ (Hexanes:EtOAc 3:1); $[\alpha]_{\mathrm{D}}-156.9\left(c 1.2, \mathrm{CHCl}_{3}\right) ; \mathrm{IR}\left(\mathrm{CH}_{2} \mathrm{Cl}_{2}\right) \vee 3060$, 2918, 2847, 1674, 1610, 1594, 1510, 1490, 1449, 1345, 1291, 1246, 1159, 1094, 1072, 1027, 843, $808,752,698 \mathrm{~cm}^{-1} ;{ }^{1} \mathrm{H}$ MR $\left(300 \mathrm{MHz}, \mathrm{CDCl}_{3}\right) \delta$ 7.61-7.56 (m, 2H), 7.32-7.05 (m, 10H), 7.09-6.96 $(\mathrm{m}, 2 \mathrm{H}), 6.89-6.83(\mathrm{~m}, 2 \mathrm{H}), 6.74-6.61(\mathrm{bs}, 2 \mathrm{H}), 5.04(\mathrm{dm}, 1 \mathrm{H}, J=2.2 \mathrm{~Hz}), 4.99(\mathrm{dm}, 1 \mathrm{H}, J=2.2$ $\mathrm{Hz}), 4.62(\mathrm{~d}, 1 \mathrm{H}, J=7.9 \mathrm{~Hz}), 4.25(\mathrm{dm}, 1 \mathrm{H}, J=13.9 \mathrm{~Hz}), 4.00(\mathrm{ddd}, 1 \mathrm{H}, J=13.9,2.2,2.2 \mathrm{~Hz}$ ), $3.85(\mathrm{~s}, 3 \mathrm{H}), 3.72(\mathrm{~m}, 1 \mathrm{H}), 2.41(\mathrm{~s}, 3 \mathrm{H}) ;{ }^{13} \mathrm{C} \mathrm{NMR}\left(75 \mathrm{MHz}, \mathrm{CDCl}_{3}\right) \delta 170.0,159.6,144.0,142.6$, $132.8,131.8,129.8,129.1,128.5,128.5,128.4,126.6,126.3,114.0,108.5,68.0,58.1,55.6,54.6$, 21.8; Chiral HPLC: Chiralcel OD-H column, 90:10 Hexanes: $i-\mathrm{PrOH}, 1 \mathrm{~mL} / \mathrm{min}, 30{ }^{\circ} \mathrm{C}, \mathrm{t}_{1}=35.3$ $\min , \mathrm{t}_{2}=78.3 \mathrm{~min}$.

\footnotetext{
${ }^{6}$ Scott, M. E.; Han, W.; Lautens, M. Org. Lett. 2004, 6, 3309-3312.

${ }^{7}$ Davis, F. A.; Prasad, K. R.; Nolt, M. B.; Wu, Y. Org. Lett. 2003, 5, 925-927.
} 
V. ${ }^{1} \mathrm{H}$ and ${ }^{13} \mathrm{C}$-NMR spectra of ring-expanded products
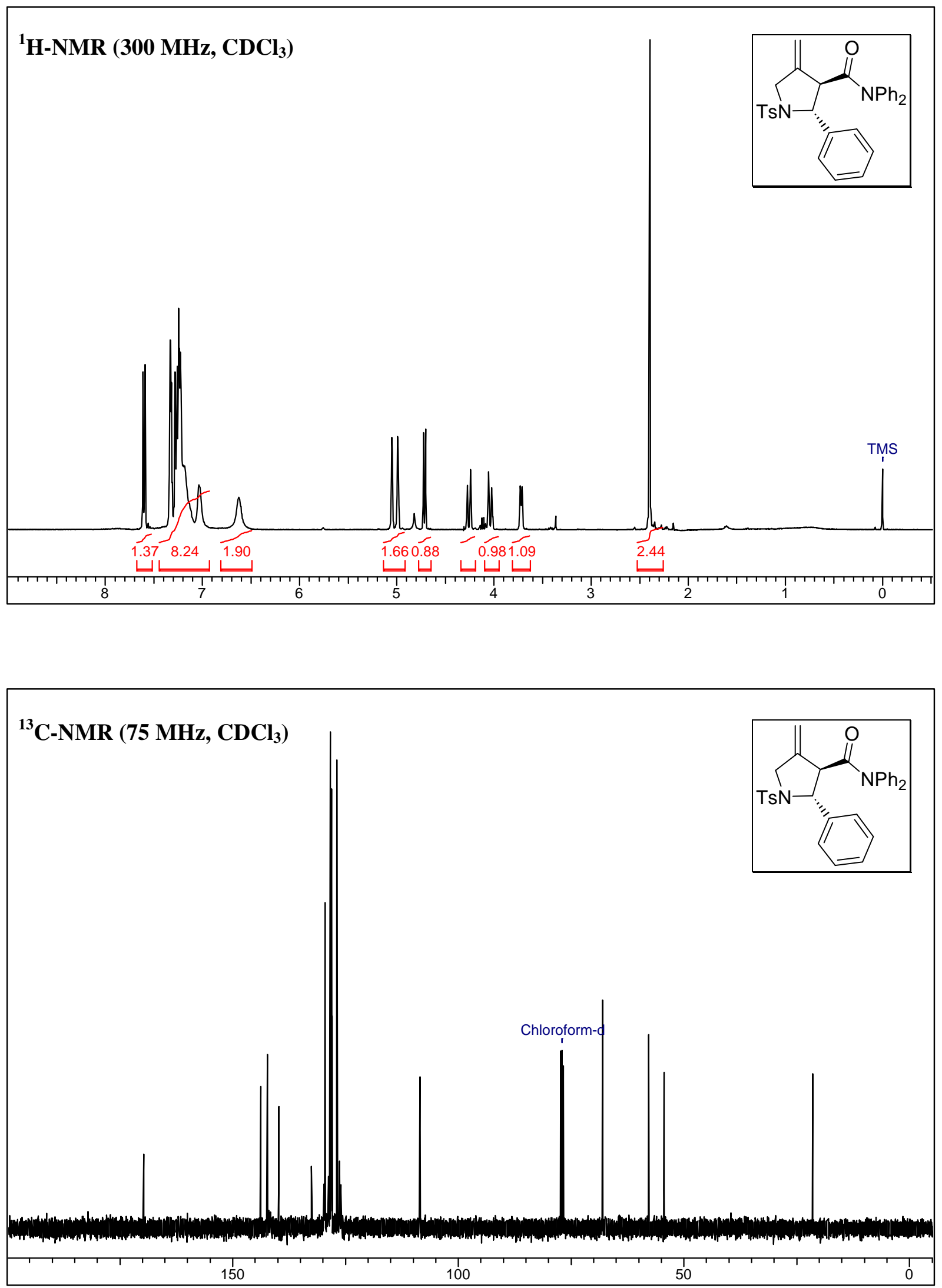

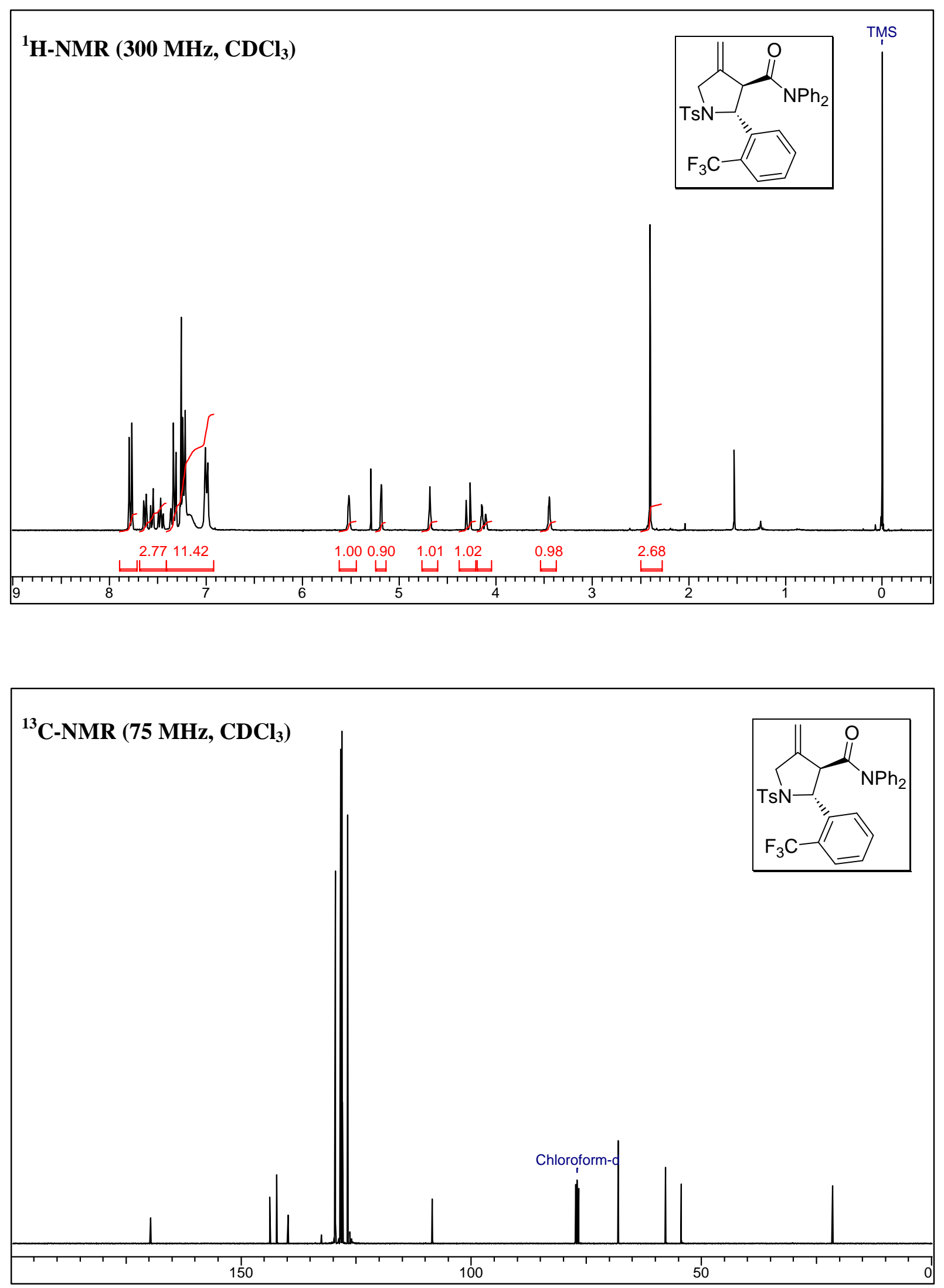

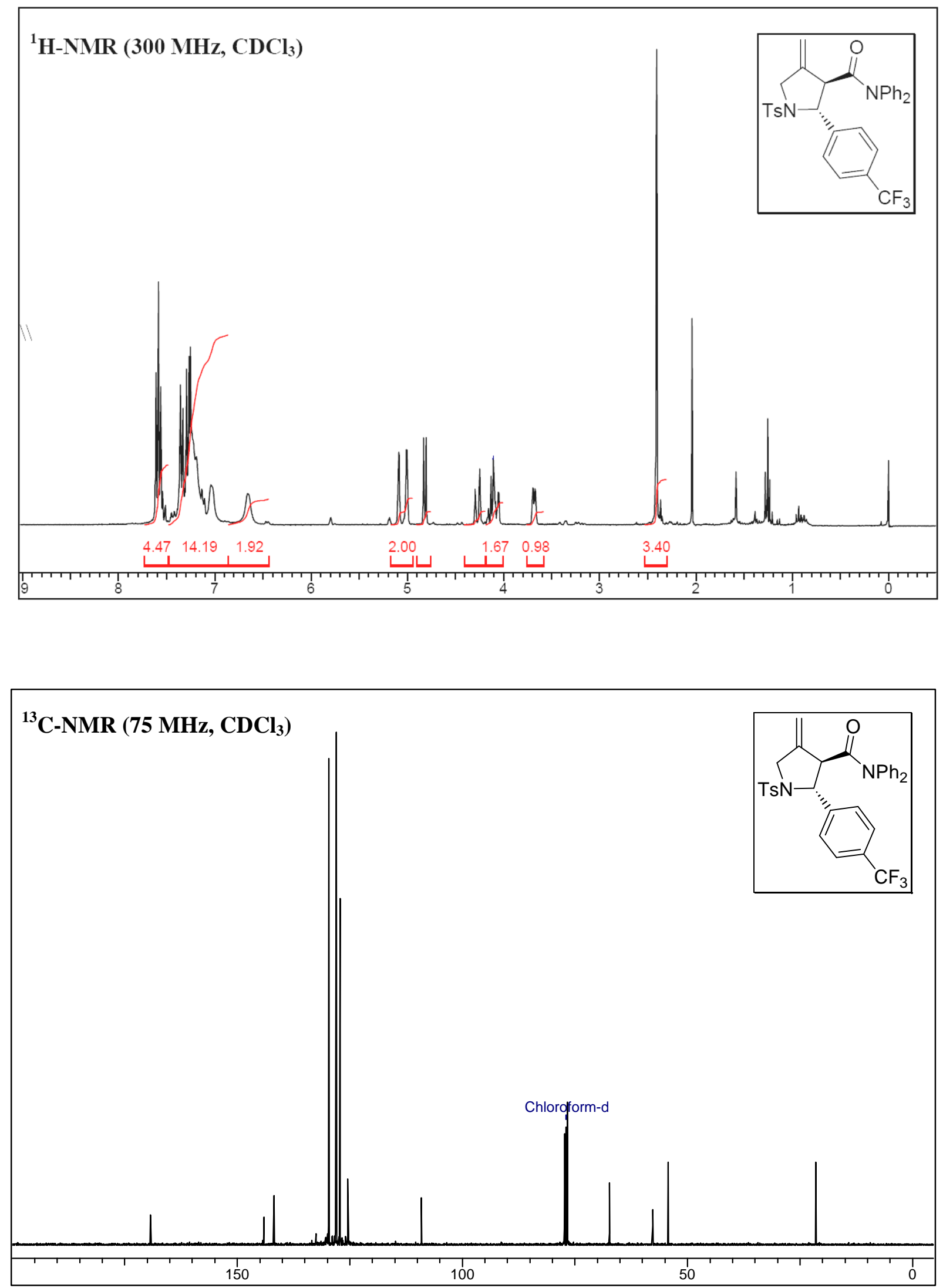

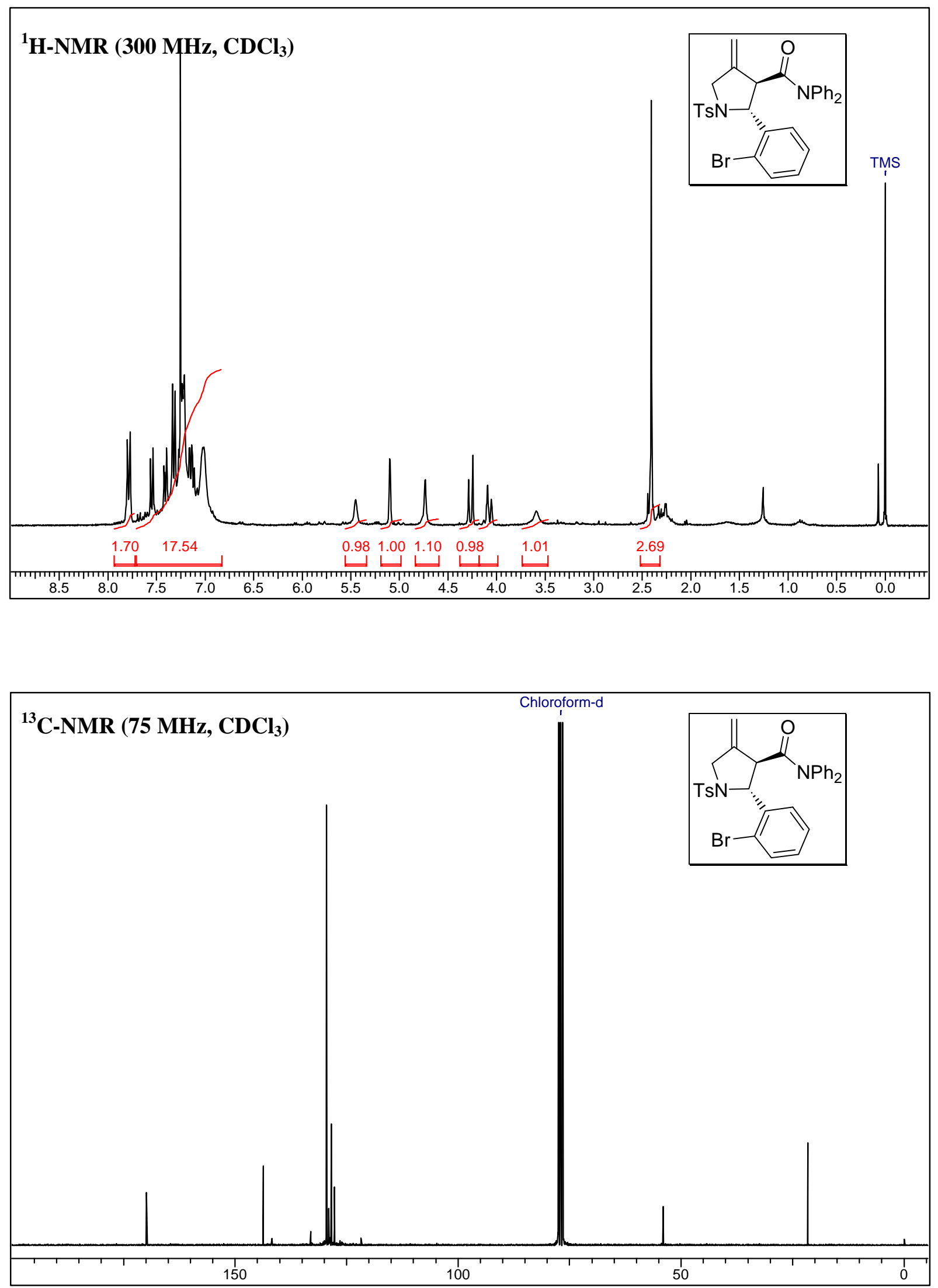

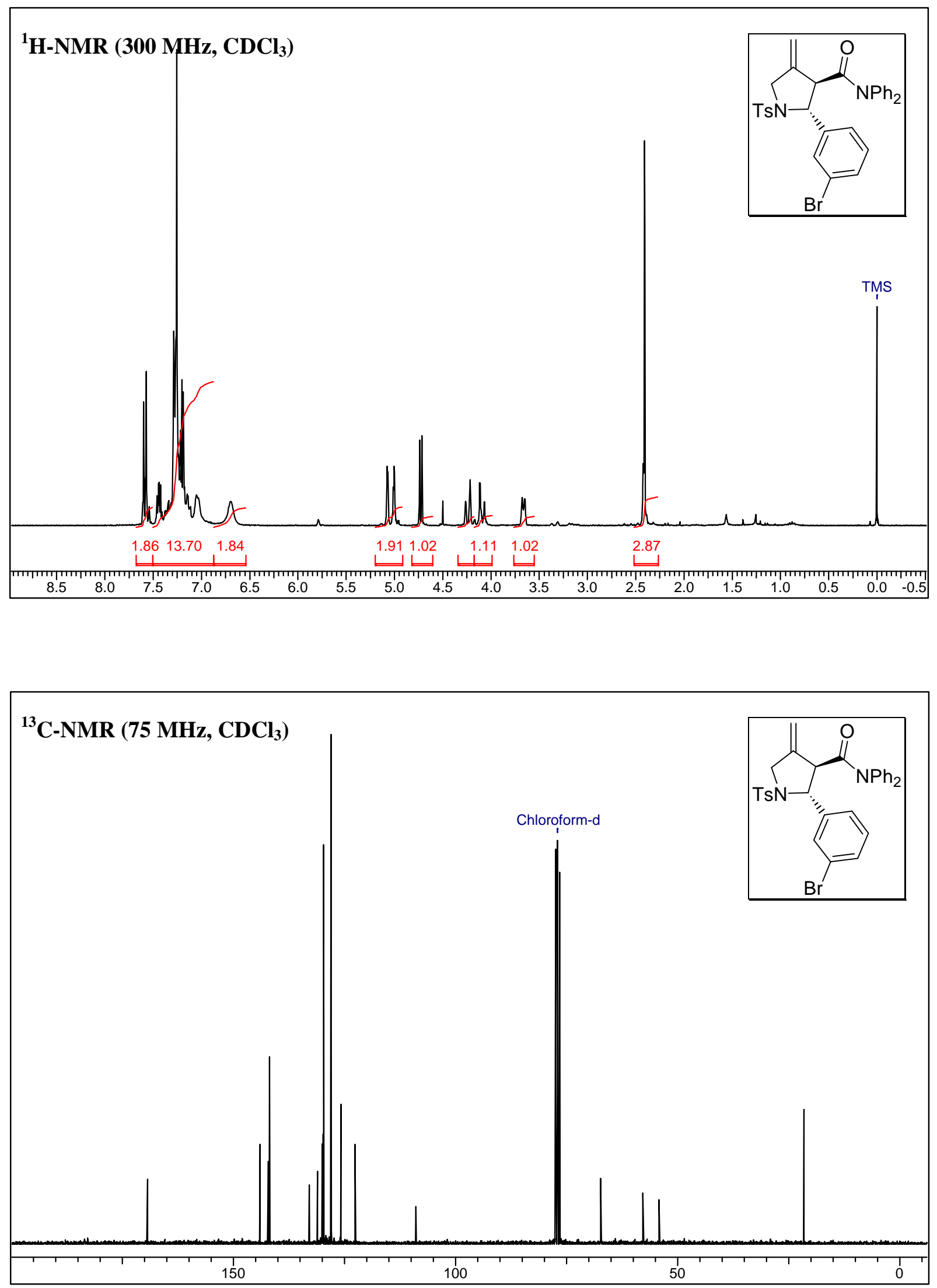
Taillier and Lautens

S18
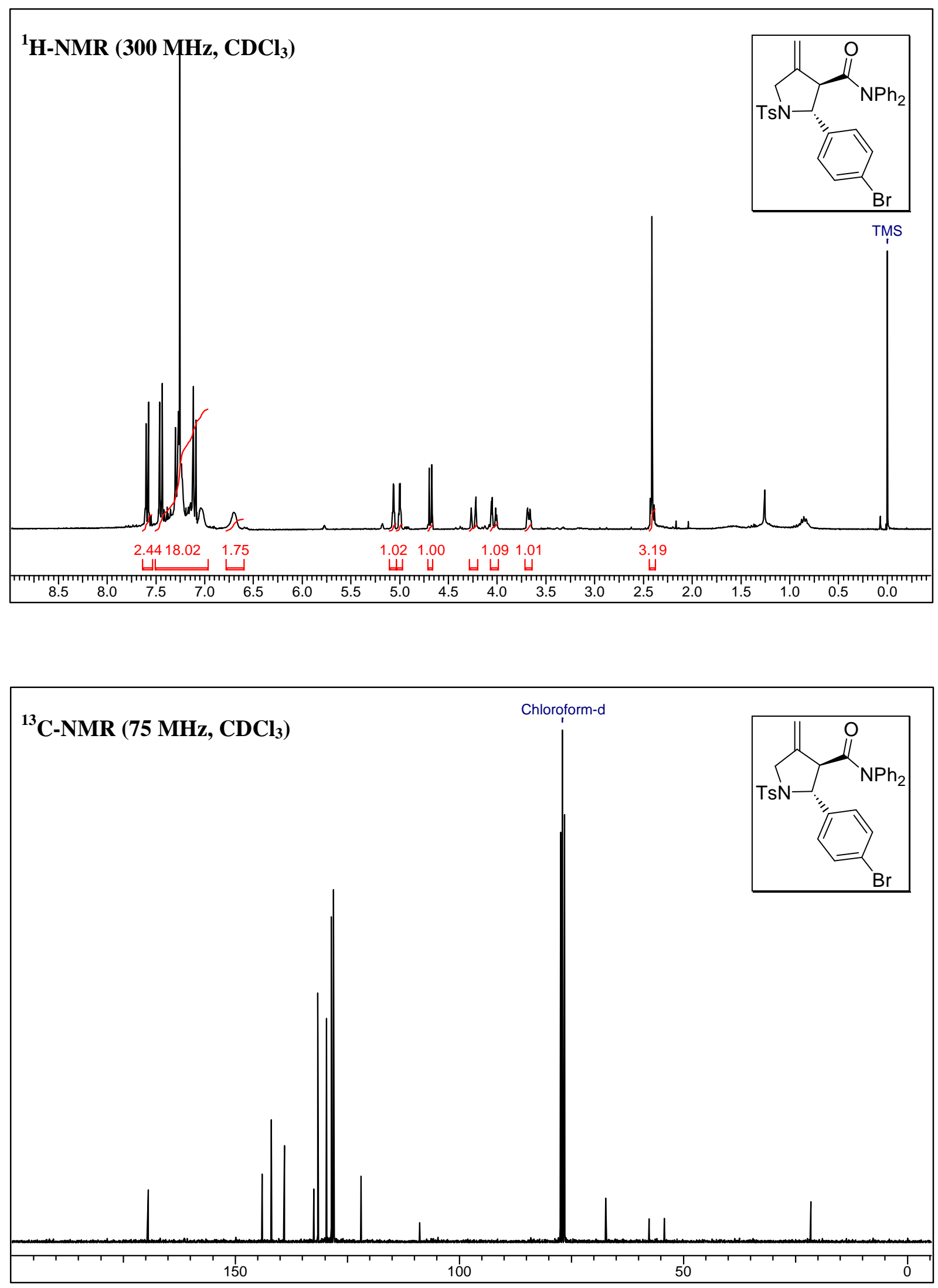

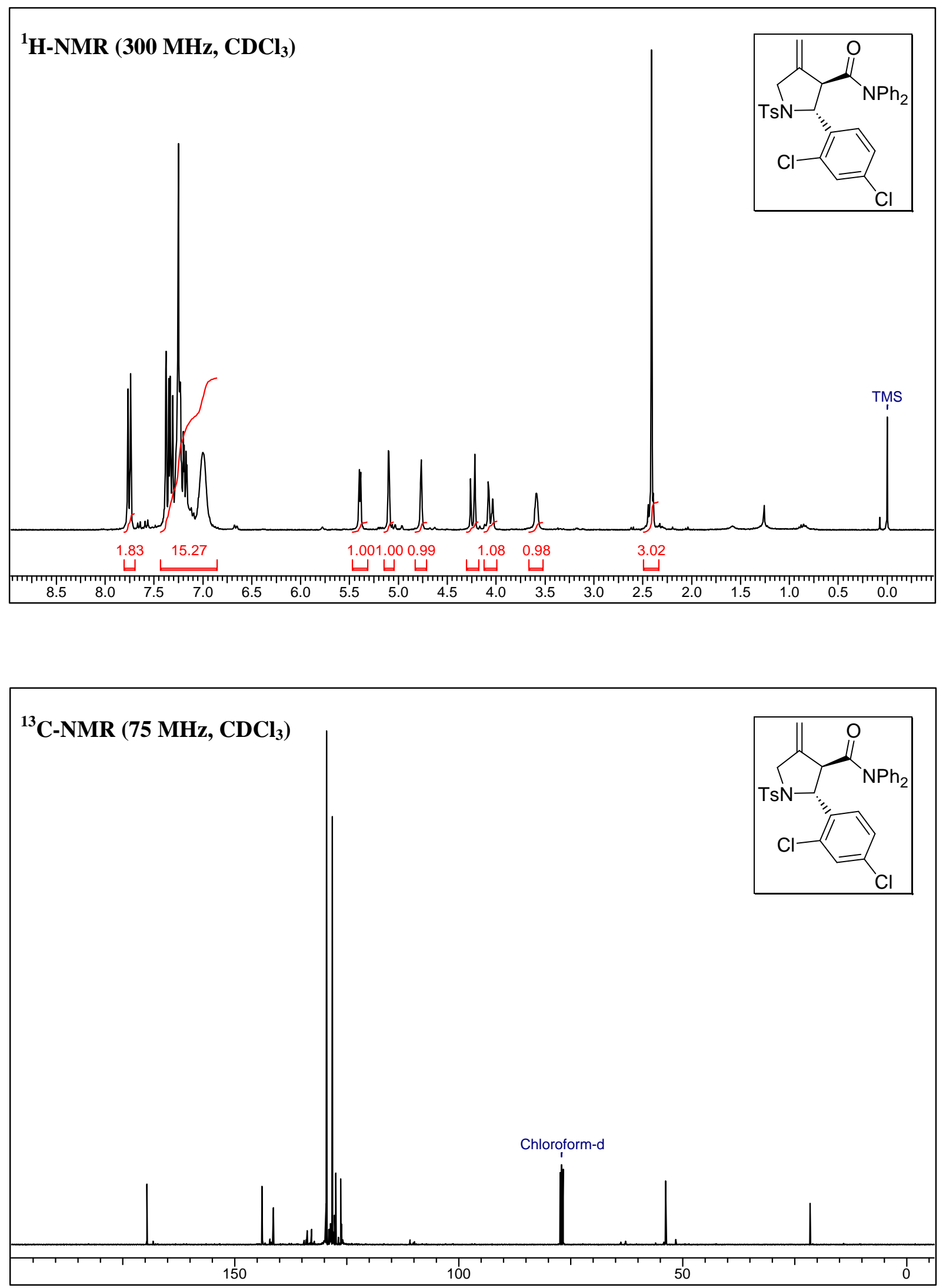

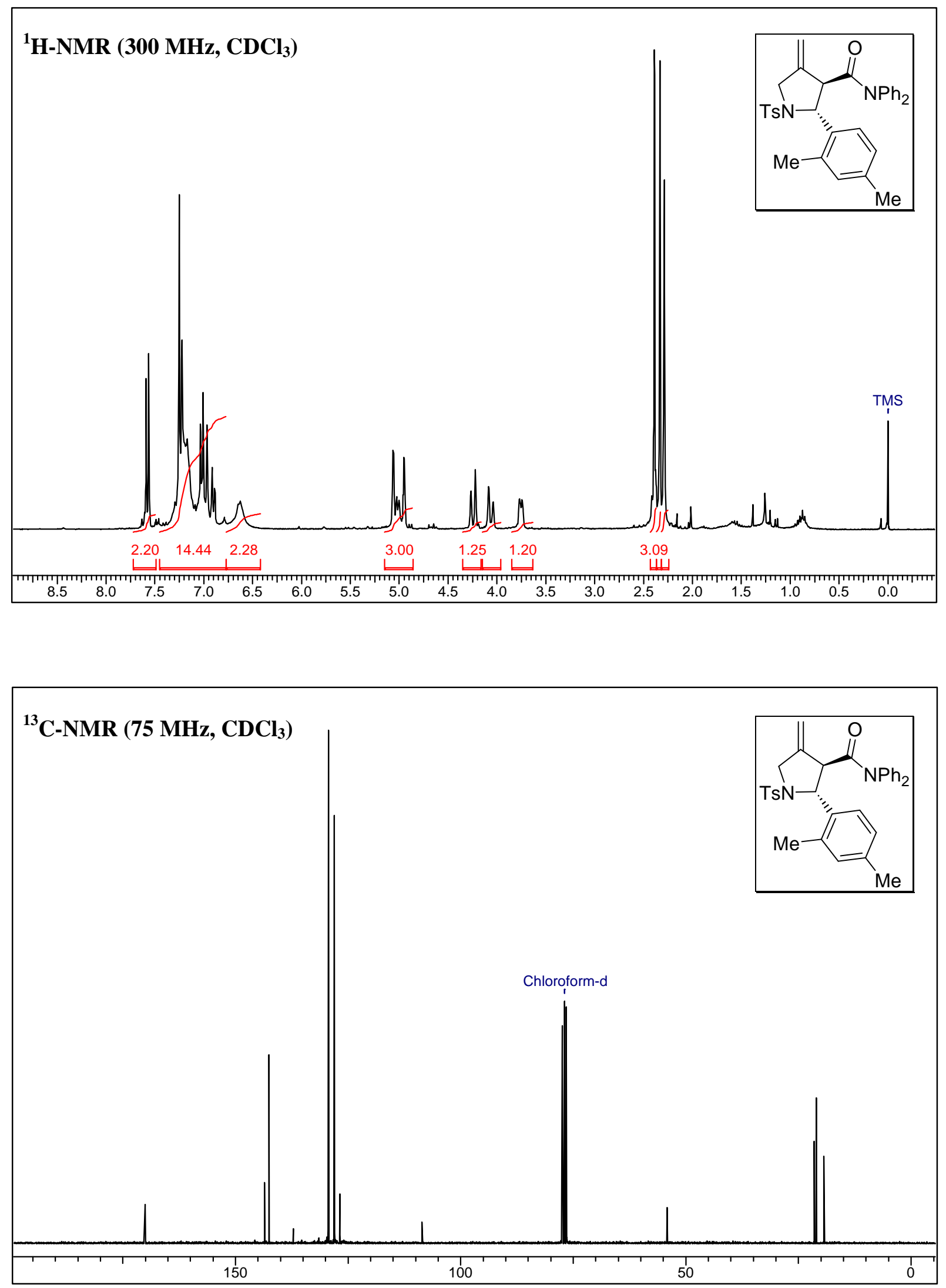

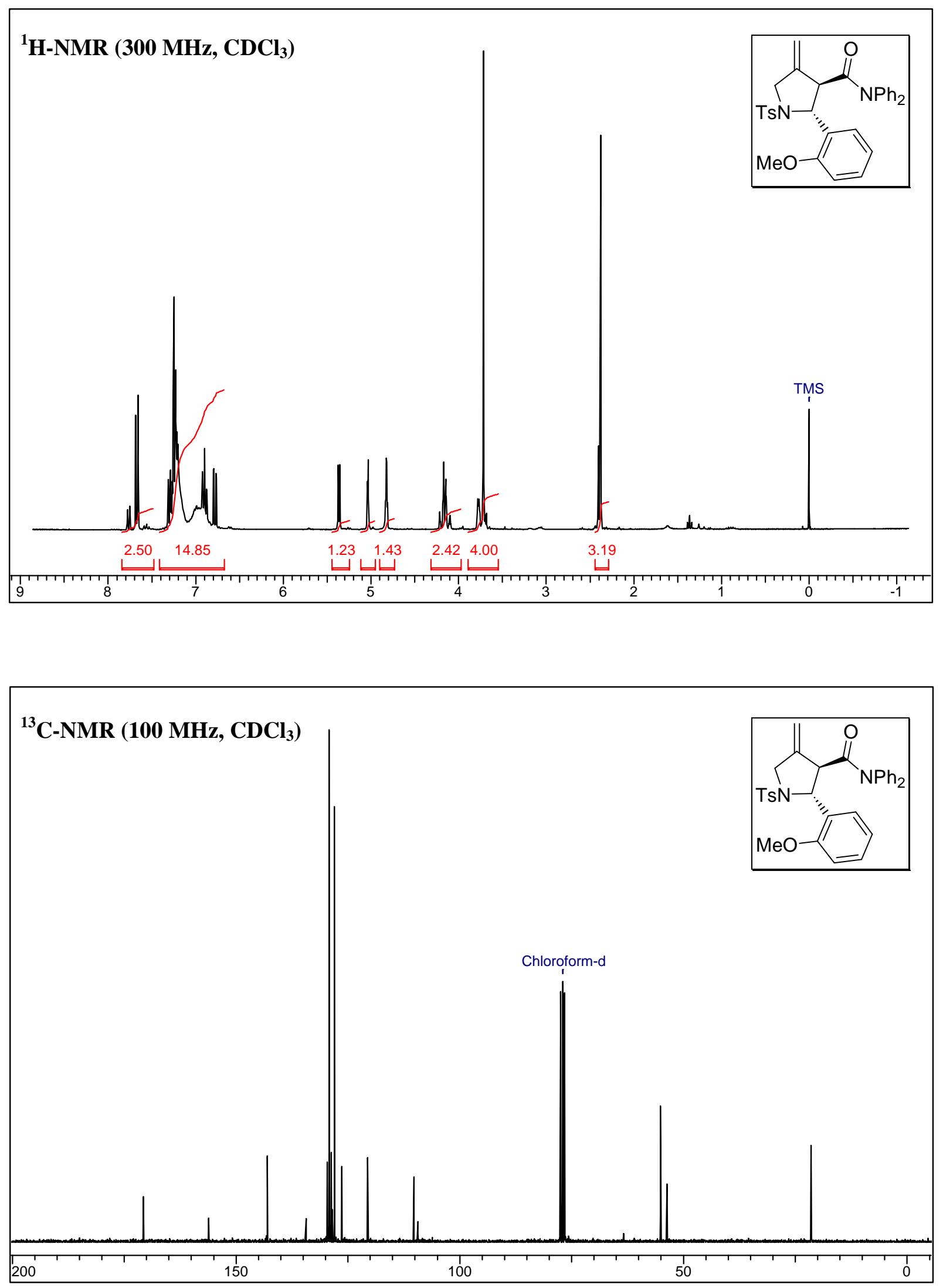

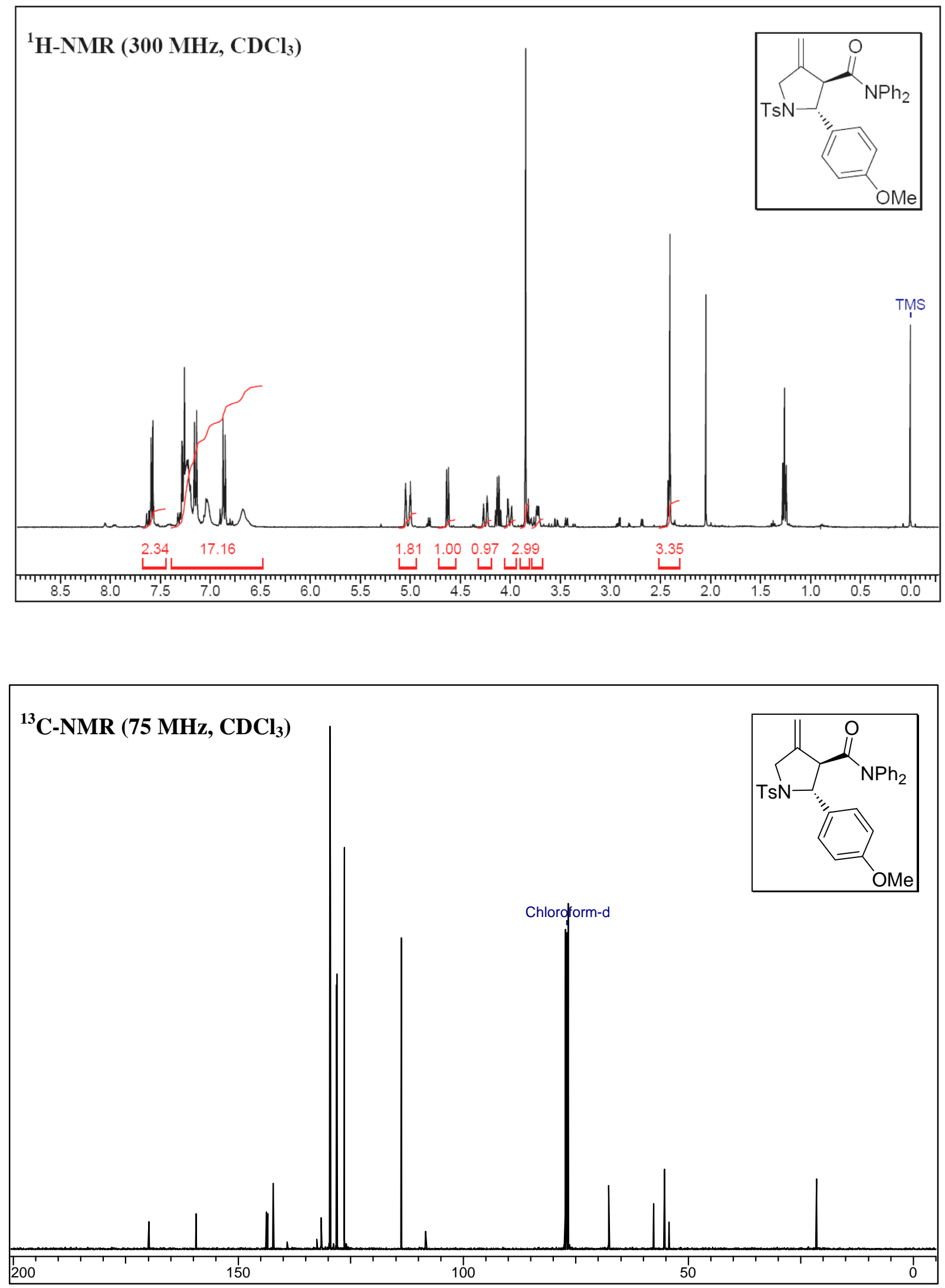


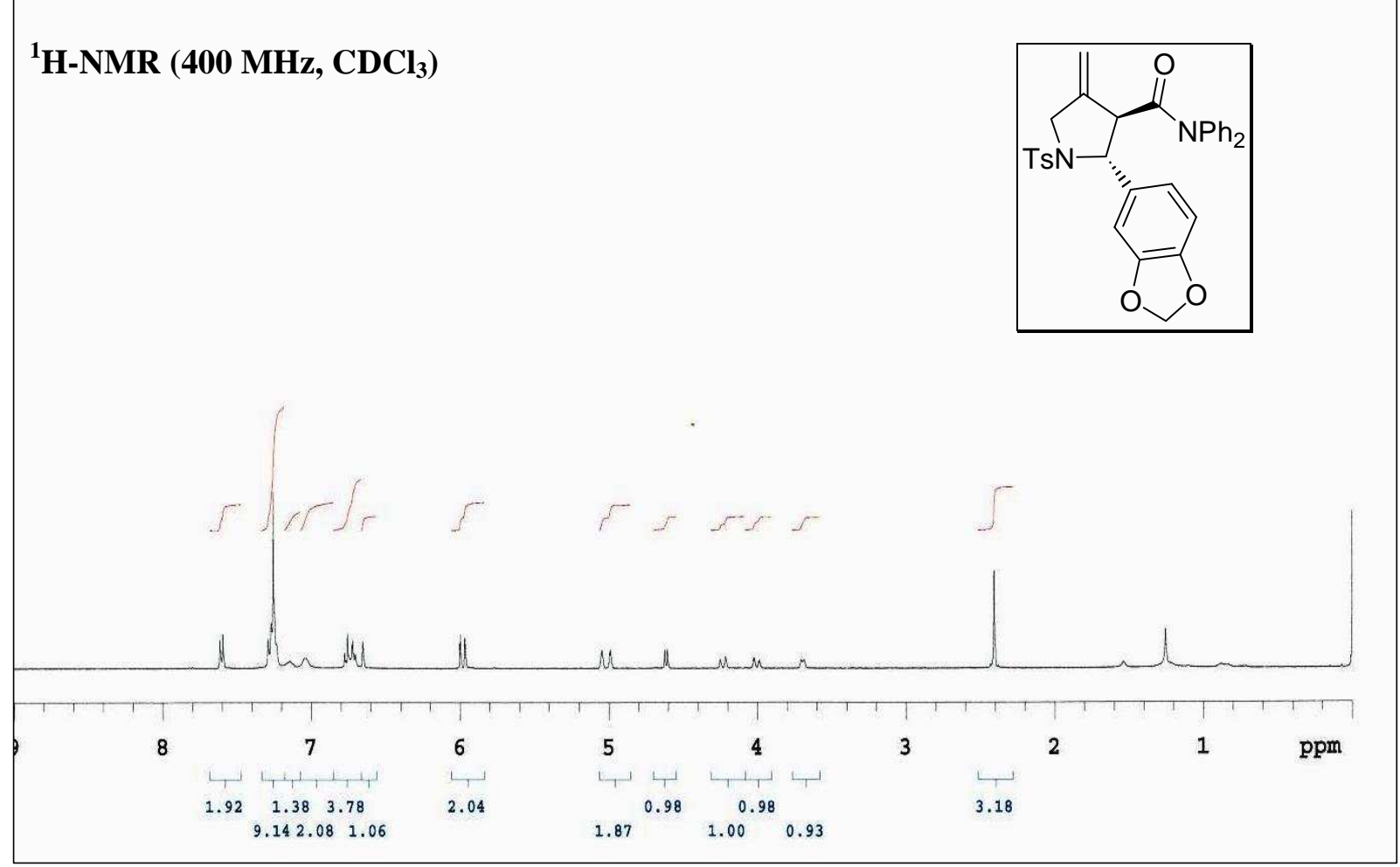



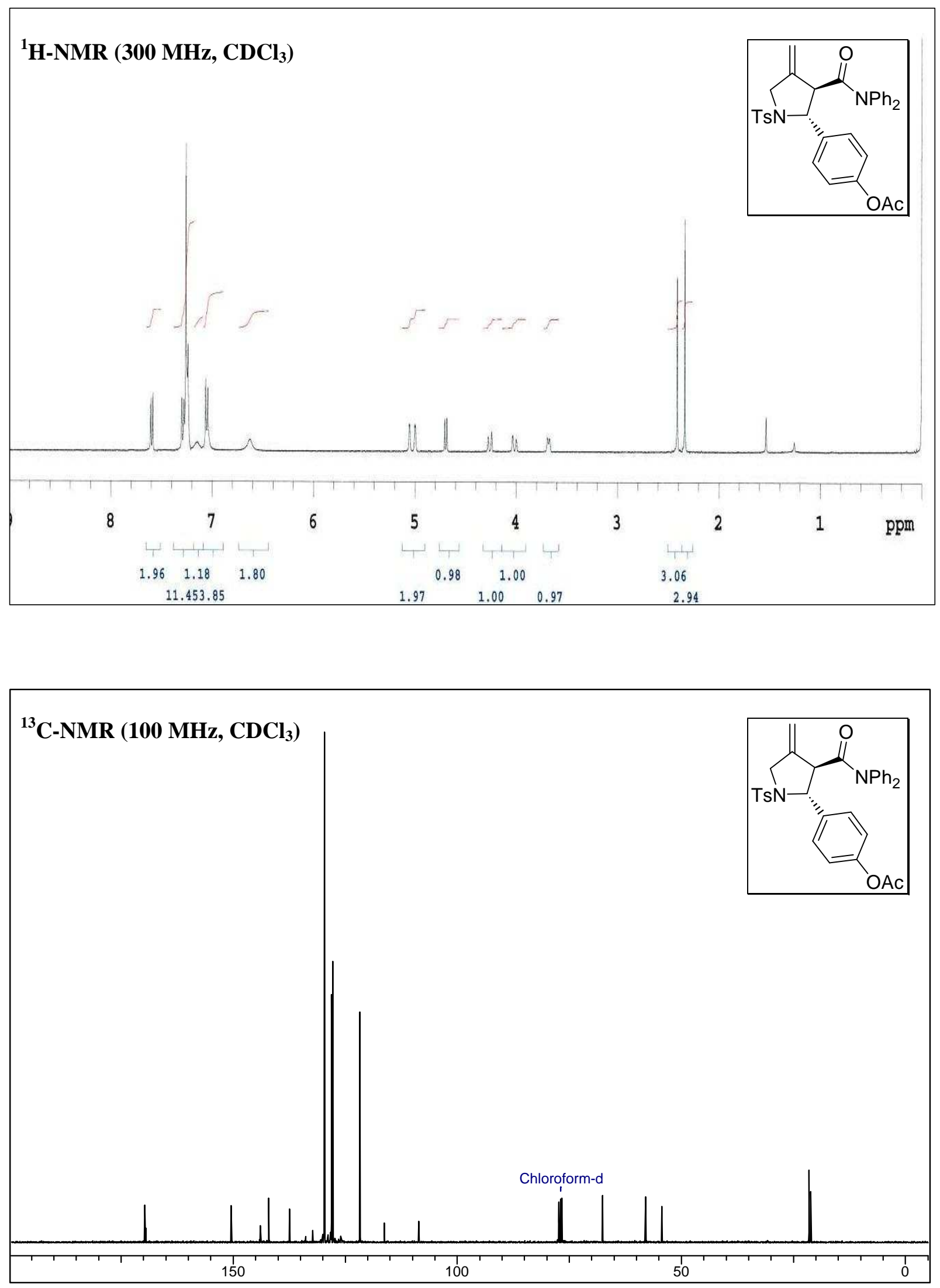

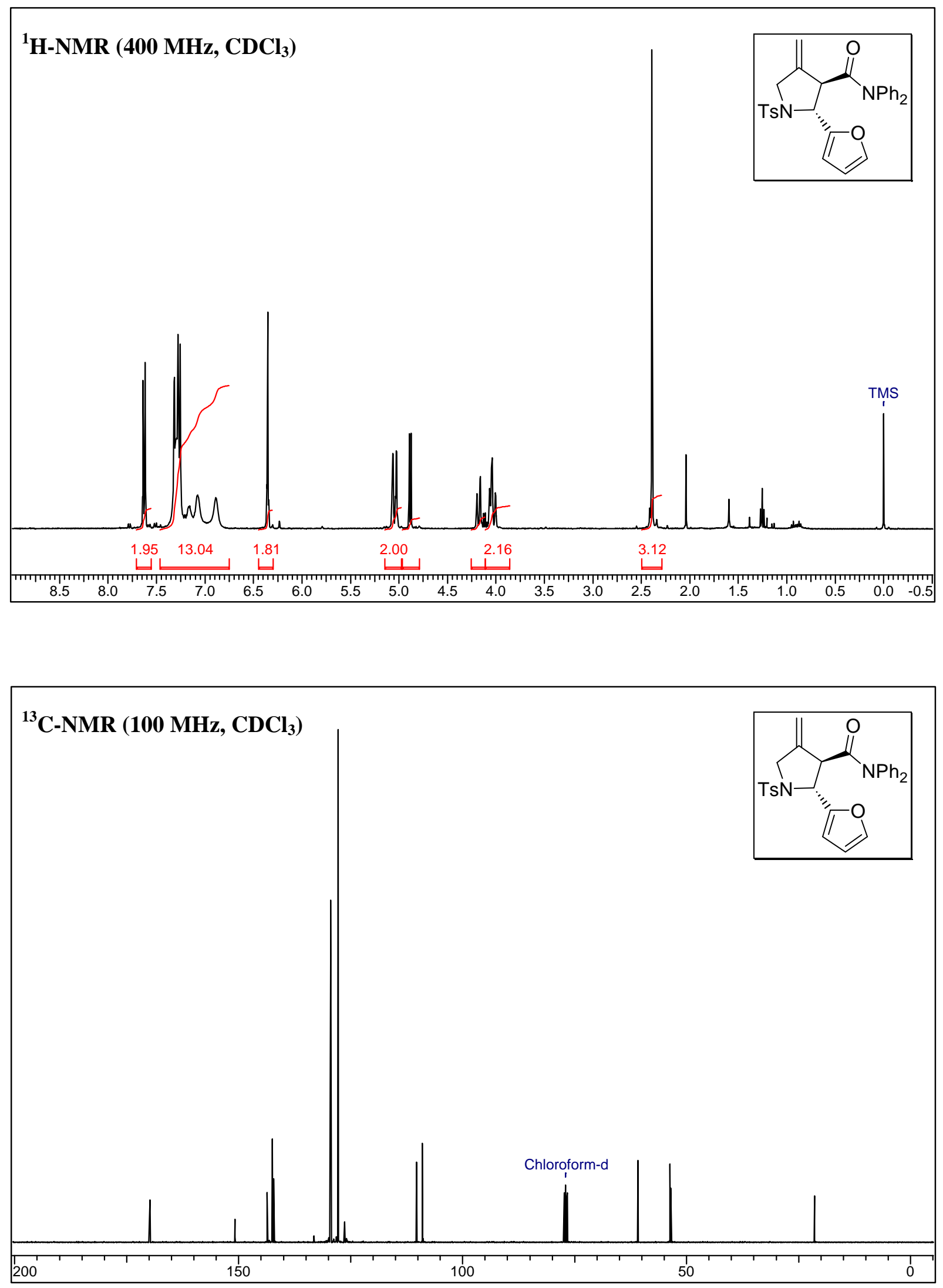

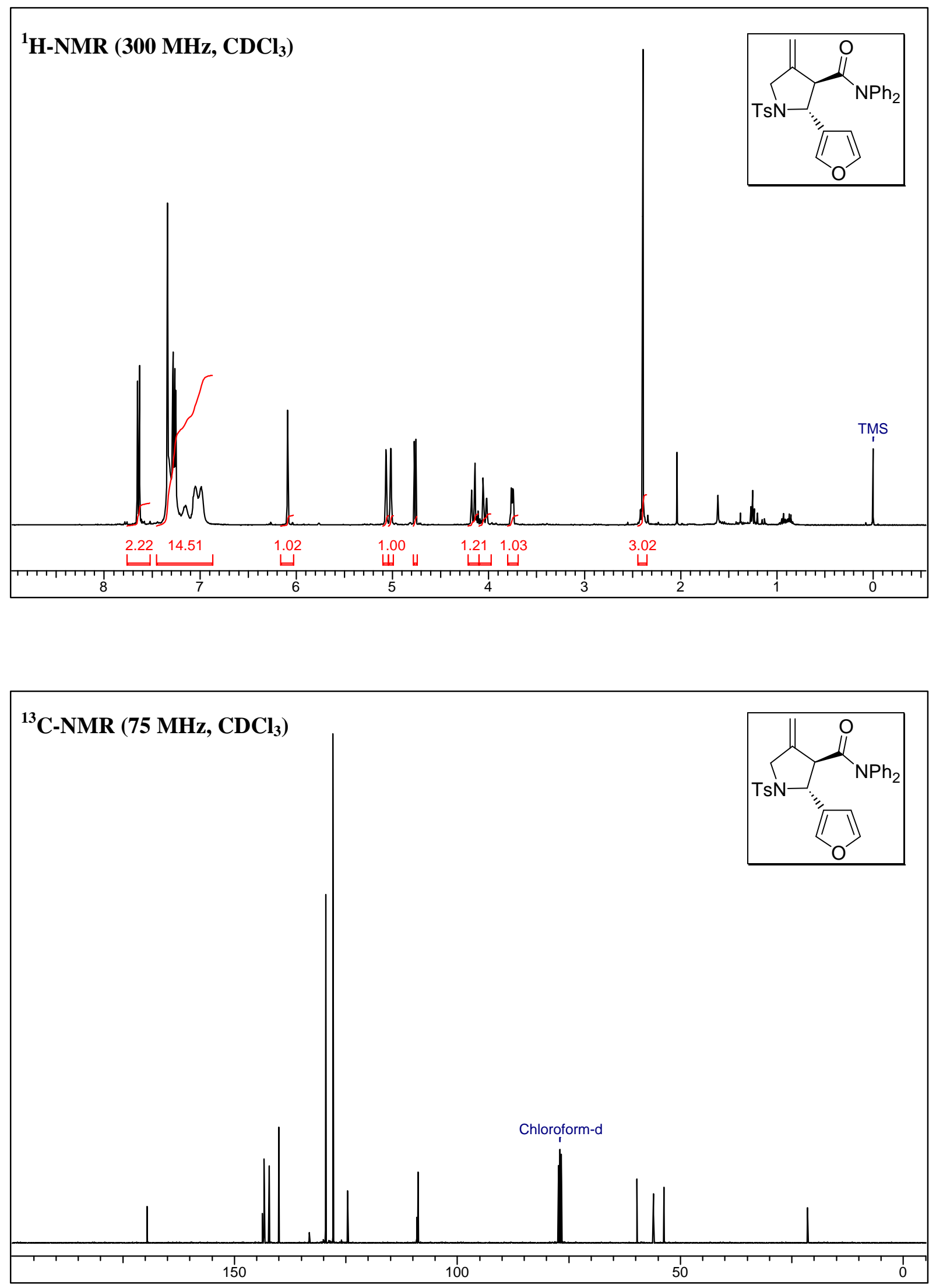

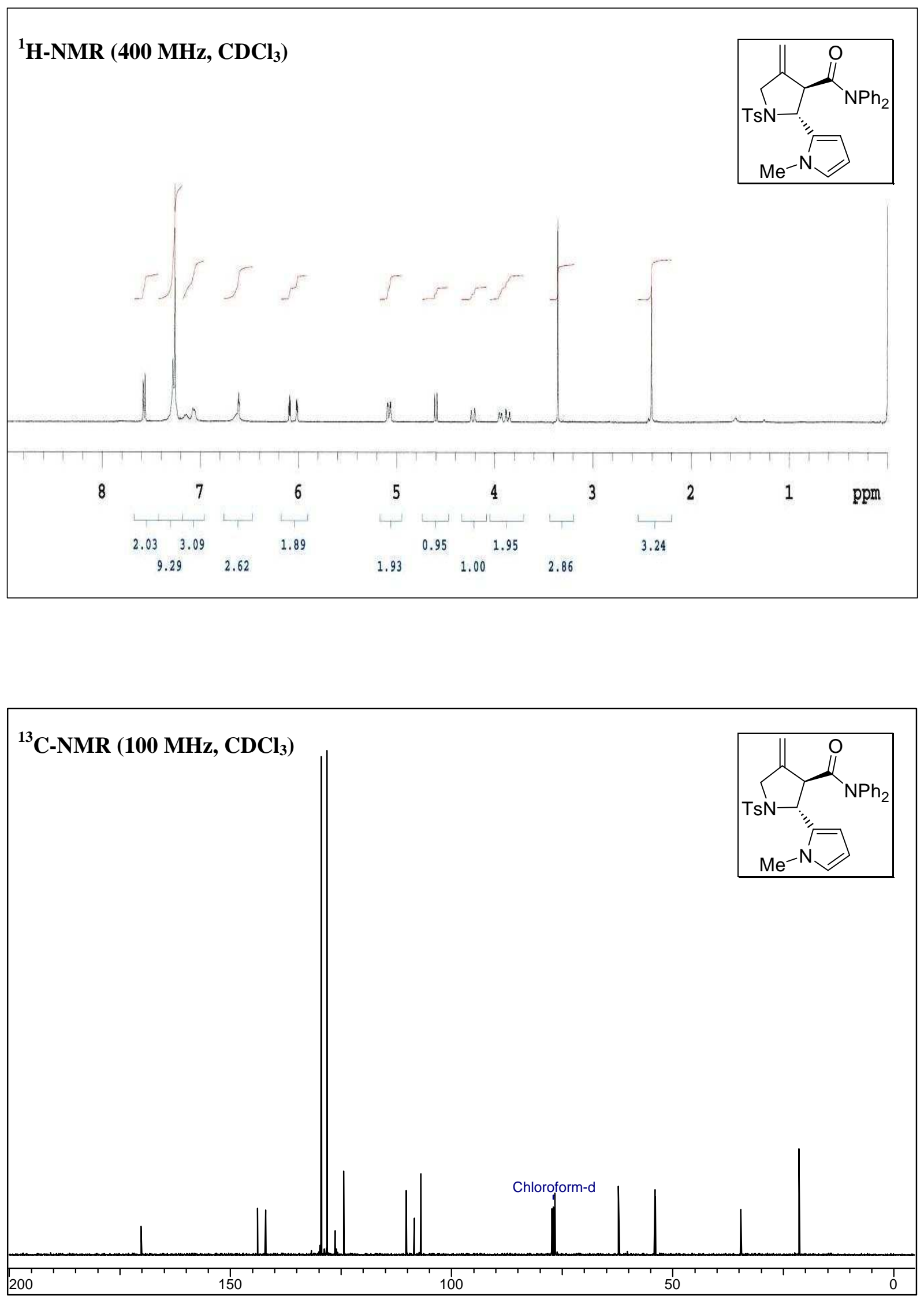

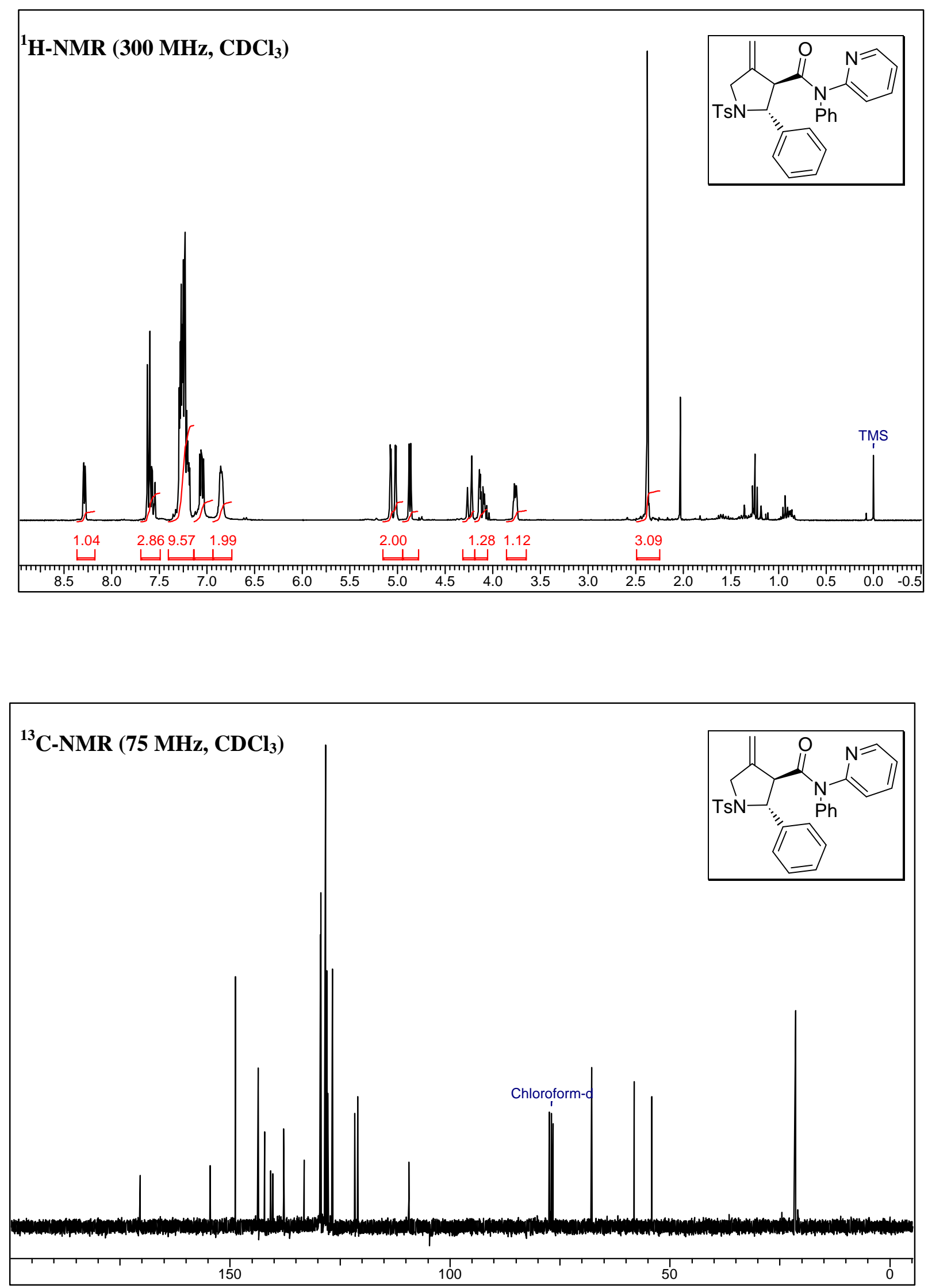


\section{HPLCs of ring-expanded products}

Chiralcel OD-H column, 94:06 Hexanes:i-PrOH, $1 \mathrm{~mL} / \mathrm{min}, 30{ }^{\circ} \mathrm{C}$.

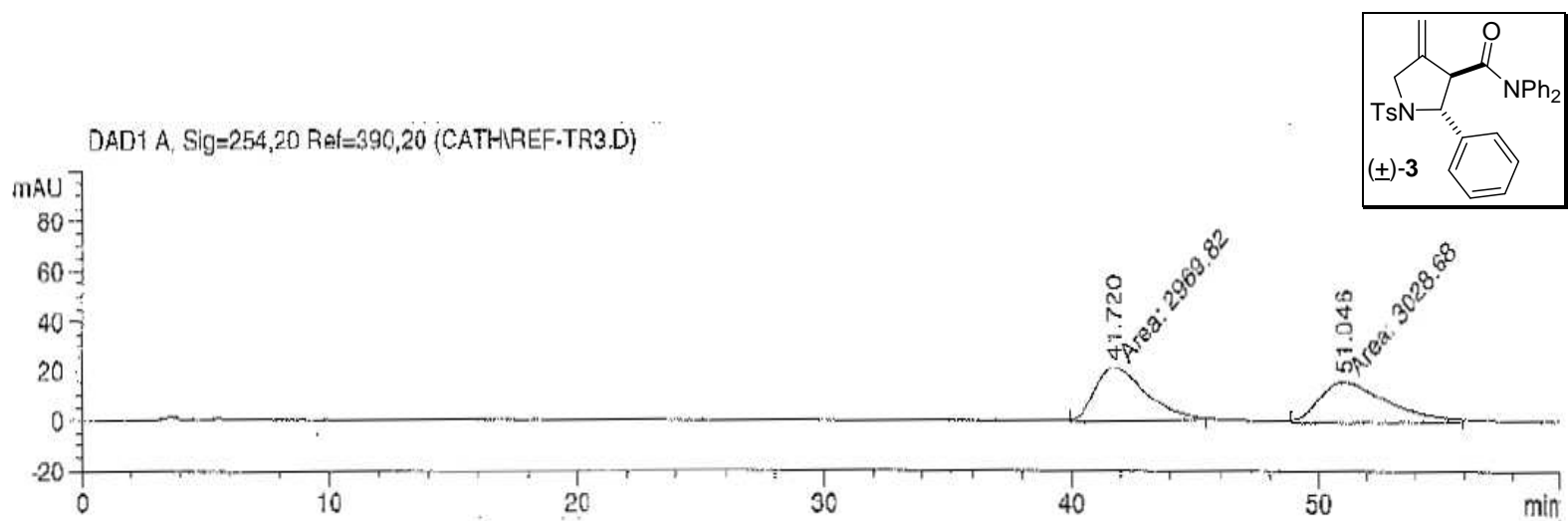

Signal 1: DAD1 A, Sigm254,20 Refm390,20

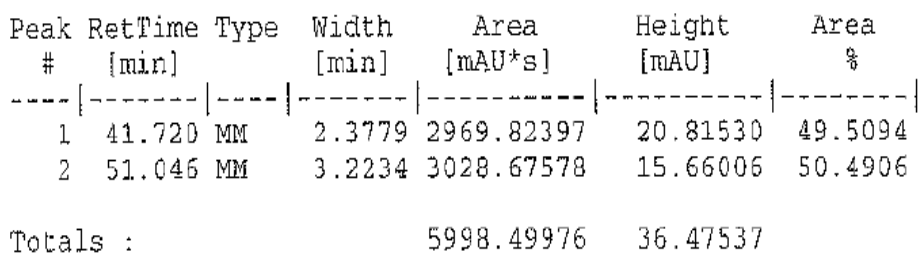

DADI A, Sig $=254,20$ Rel $=390,20$ (CATHICT-79-2B.D)

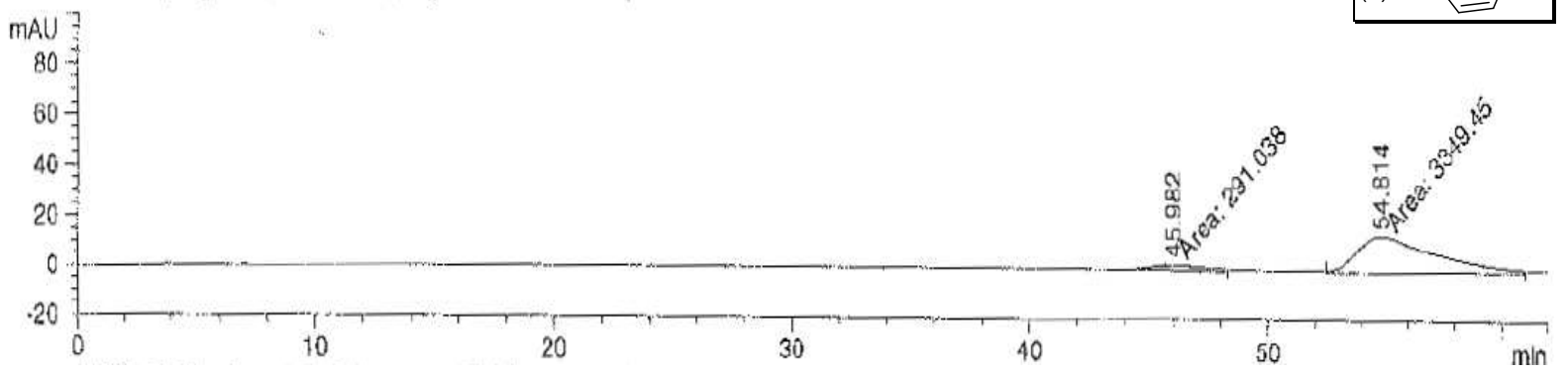

Signāl 1: DAD1 A, Sig $254,20 \operatorname{Ref}=390,20$

\begin{tabular}{|c|c|c|c|c|c|}
\hline $\begin{array}{c}\text { Peak } \\
\#\end{array}$ & $\begin{array}{l}\text { RetTime Type } \\
\text { [min] }\end{array}$ & $\begin{array}{l}\text { Width } \\
\text { [min] }\end{array}$ & $\begin{array}{c}\text { Area } \\
{\left[\mathrm{mAU}{ }^{*} \mathrm{~s}\right]}\end{array}$ & $\begin{array}{l}\text { Height } \\
\text { [mAU] }\end{array}$ & $\begin{array}{c}\text { Area } \\
\text { 뭄 }\end{array}$ \\
\hline & , & & & & r-r-an \\
\hline 1 & $45.982 \mathrm{MM}$ & 2.4896 & 291.03809 & 1.94832 & $7.9945^{\circ}$ \\
\hline 2 & $54.814 \mathrm{MM}$ & 3.9053 & 3349.44678 & 14.29427 & 92.0055 \\
\hline ta & & & 3640.48486 & 4259 & \\
\hline
\end{tabular}


Chiral HPLC: Chiralcel OD-H column, 94:06 Hexanes:i-PrOH, $1 \mathrm{~mL} / \mathrm{min}, 30{ }^{\circ} \mathrm{C}$.

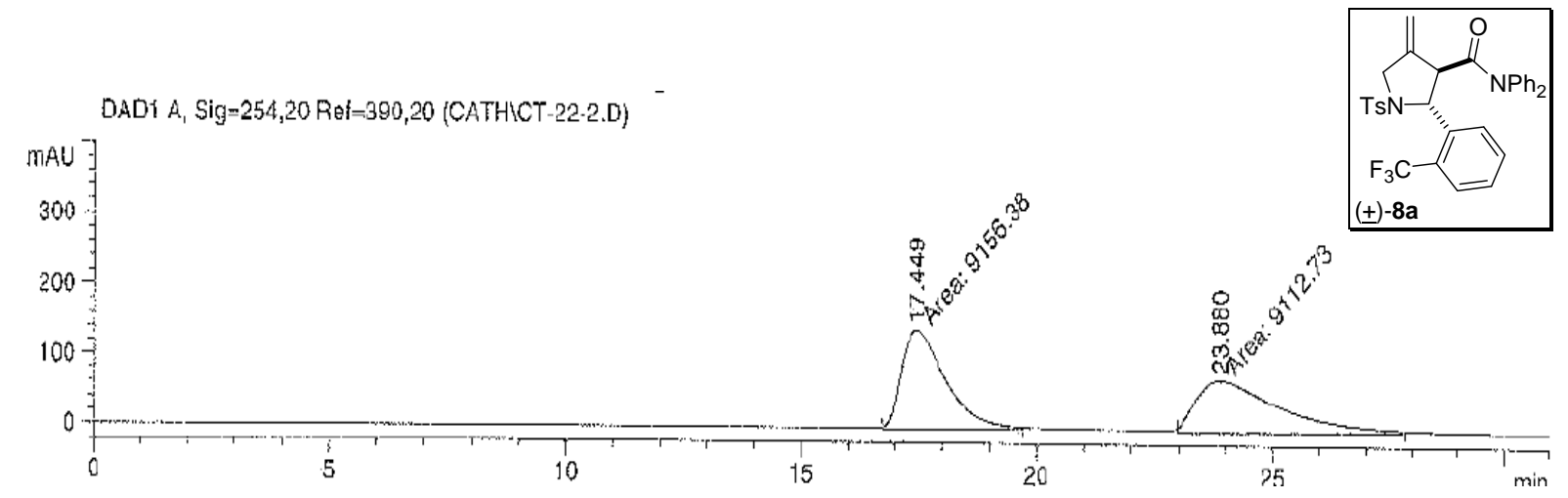

Signal 1: DAD.1 A, Sig=254,20 Ref $=390,20$

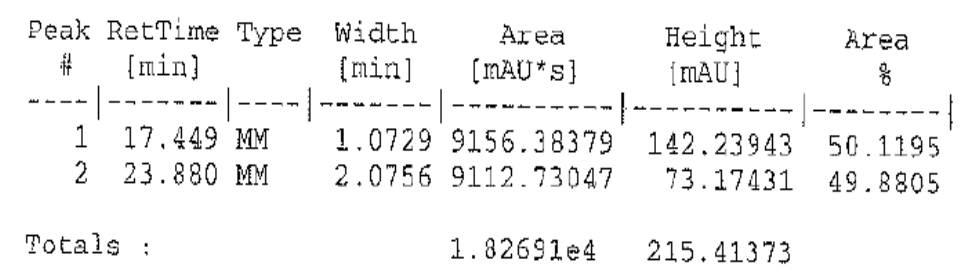

DAD1 A, Sig=254,20 Ref $=390,20$ (CATHLCT-101-2.D)

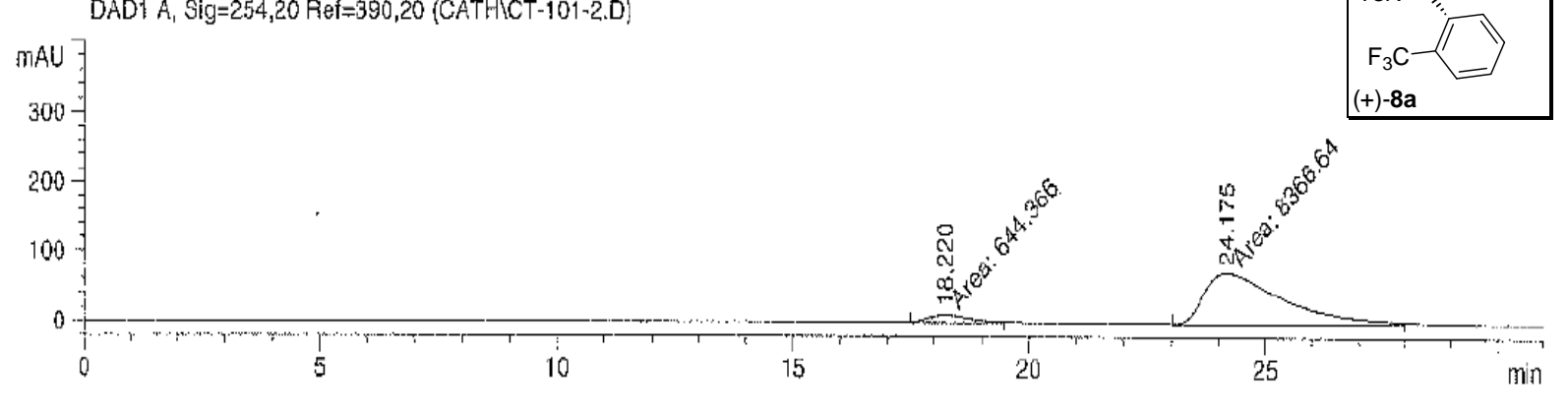

Signal 1: DAD1 A, Sig=254,20 Ref $=390,20$

\begin{tabular}{|c|c|c|c|c|c|}
\hline $\begin{array}{c}\text { Peak } \\
\text { \# }\end{array}$ & $\begin{array}{l}\text { RetTime Type } \\
\text { [min] }\end{array}$ & $\begin{array}{l}\text { Width } \\
\text { [min] }\end{array}$ & $\begin{array}{c}\text { Area } \\
{\left[\mathrm{MAJ}^{*} \mathrm{~S}\right]}\end{array}$ & $\begin{array}{l}\text { Height } \\
\text { [mat] }\end{array}$ & $\begin{array}{c}\text { Area } \\
\%\end{array}$ \\
\hline & & & & & 7.500 \\
\hline 1 & $18.220^{\circ} \mathrm{MM}$ & 1.0534 & 644.36615 & 10.19550 & 7.2509 \\
\hline 2 & $24.175 \mathrm{MM}$ & 1.93 & 8366.64160 & 72.13831 & 92.8491 \\
\hline $\mathrm{ta}$ & & & 9011.00775 & 381 & \\
\hline
\end{tabular}




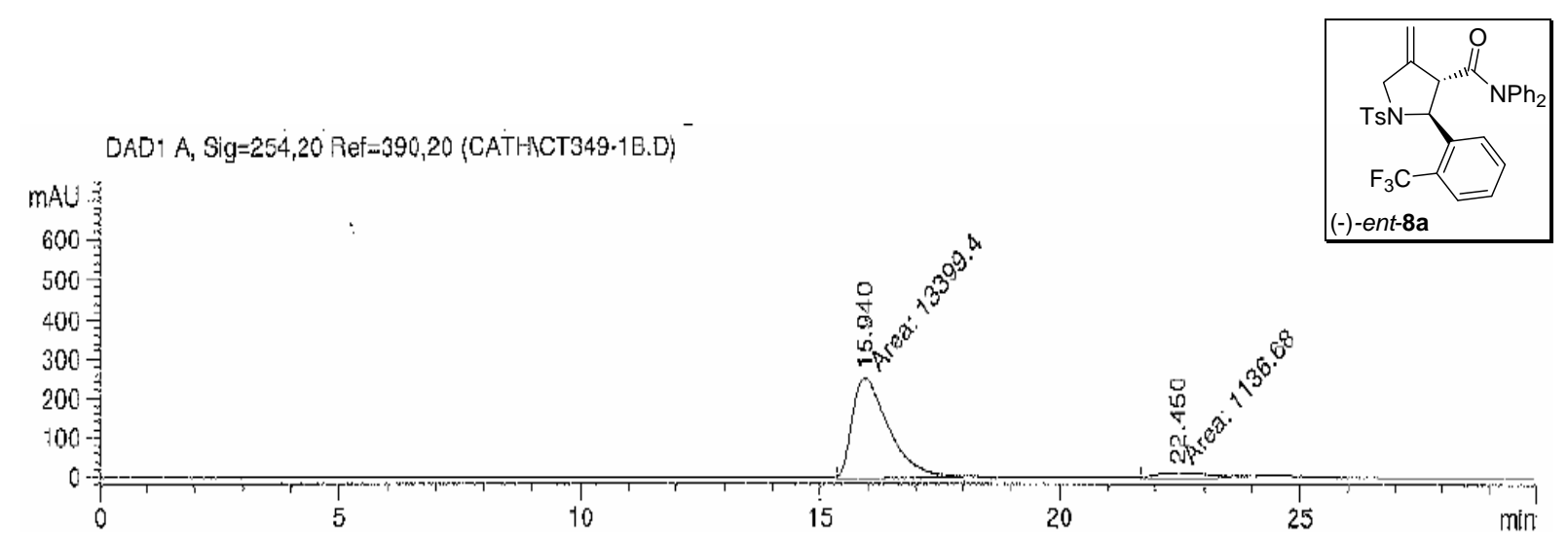

Signal 1: DADI A, Sig=254,20 ReE $=390,20$

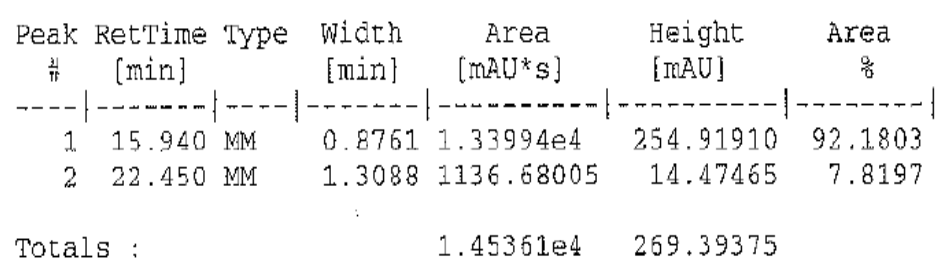


Chiralpak AD column, 95:05 Hexanes:i-PrOH, $1 \mathrm{~mL} / \mathrm{min}, 30{ }^{\circ} \mathrm{C}$.

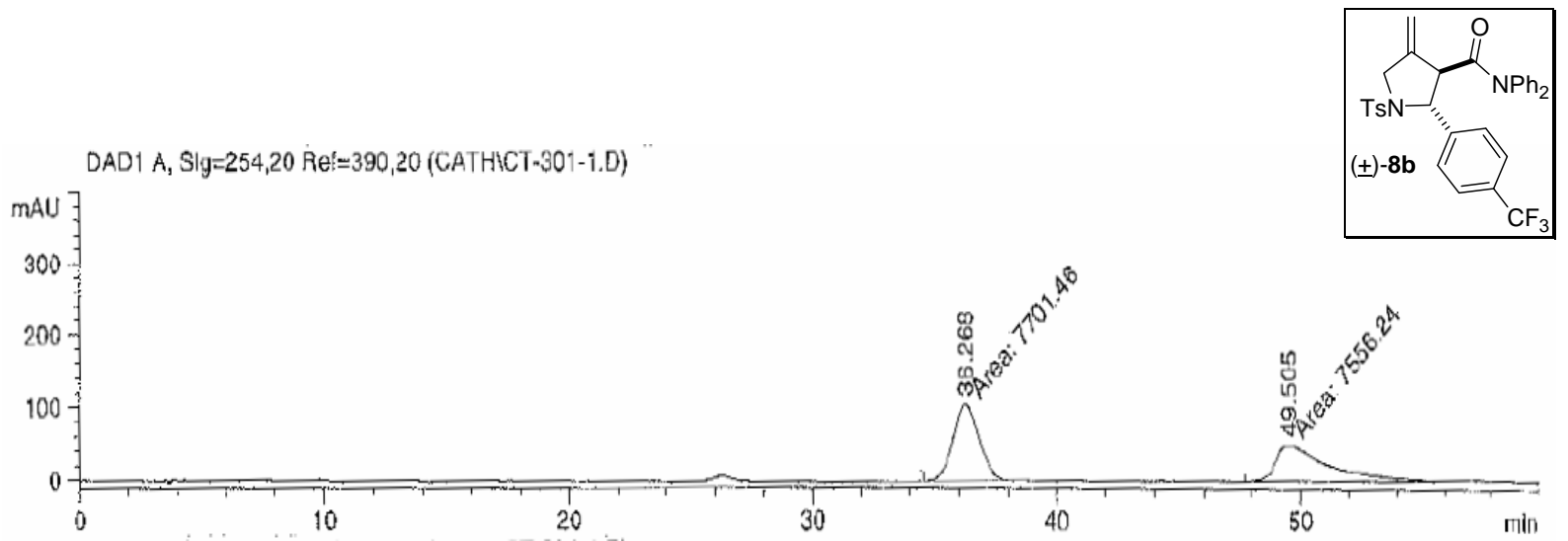

Signal 1: DAD1 A, Sig=254,20 Ref $=390,20$

\begin{tabular}{|c|c|c|c|c|c|}
\hline $\begin{array}{c}\text { Peak } \\
\quad \#\end{array}$ & $\begin{array}{l}\text { RetTime Type } \\
\text { [min] }\end{array}$ & $\begin{array}{l}\text { Width } \\
\text { [min] }\end{array}$ & $\begin{array}{c}\text { Area } \\
{\left[\mathrm{MAU}^{*} s\right]}\end{array}$ & $\begin{array}{l}\text { Height } \\
\text { [mAU] }\end{array}$ & $\begin{array}{c}\text { Area } \\
:\end{array}$ \\
\hline- & & & & & \\
\hline 2 & $\begin{array}{l}58 \mathrm{MM} \\
05 \mathrm{MM}\end{array}$ & $\begin{array}{l}1.2399 \\
2.5156\end{array}$ & $\begin{array}{l}7701.46240 \\
7556.24170\end{array}$ & $\begin{array}{r}103.52328 \\
50.06348\end{array}$ & 49.5241 \\
\hline
\end{tabular}

Totals : $\quad 1.52577 \mathrm{e} 4 \quad 153.58676$

DAD1 A, Sig $=254,20$ Fef $=390,20$ (CATHICT-307-1.D)

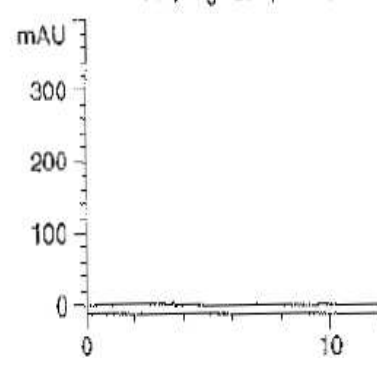

Signal 1: DAD1 A, Sig=254,20 Ref $=390,20$

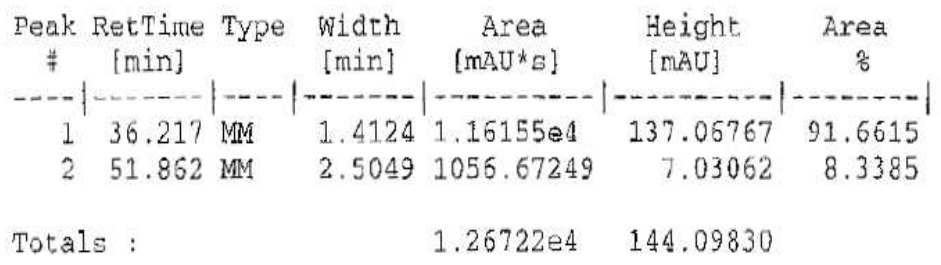


Chiralpak AD column, 95:05 Hexanes:i-PrOH, $1 \mathrm{~mL} / \mathrm{min}, 30{ }^{\circ} \mathrm{C}$.

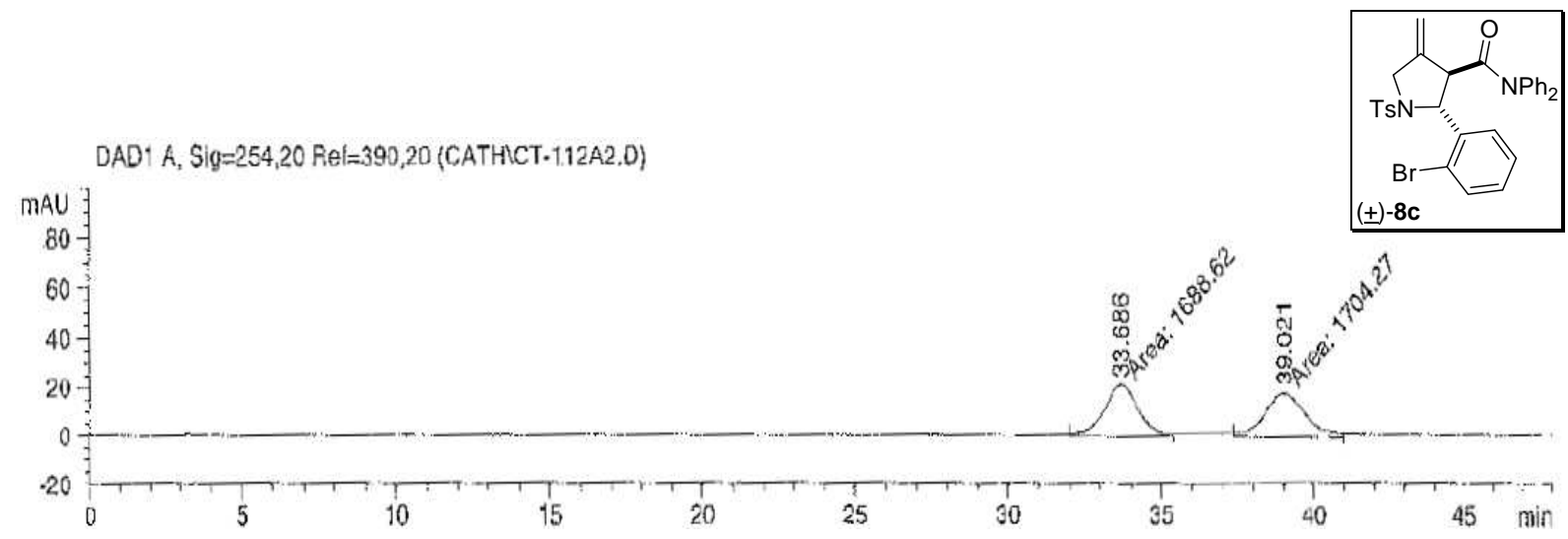

Signal 1: DAD1 A, Sig=254, 20 Ref $=390,20$

\begin{tabular}{|c|c|c|c|c|c|}
\hline eak & $\begin{array}{l}\text { RetTime Type } \\
\text { [min] }\end{array}$ & $\begin{array}{l}\text { Width } \\
\text { [min] }\end{array}$ & $\begin{array}{c}\text { Area } \\
{\left[\mathrm{mAU}^{*} \mathrm{~s}\right]}\end{array}$ & $\begin{array}{l}\text { Height } \\
\text { [mAU] }\end{array}$ & $\begin{array}{c}\text { Area } \\
\frac{2}{6}\end{array}$ \\
\hline 1 & $\mathrm{MM}$ & & 1688 & 21 & \\
\hline 2 & & & 2704 & & \\
\hline & & & 3392. & 9.45338 & \\
\hline
\end{tabular}

DAD1 A, Sig $=254,20$ Ret $=390,20$ (CATHICT-114-1,D)
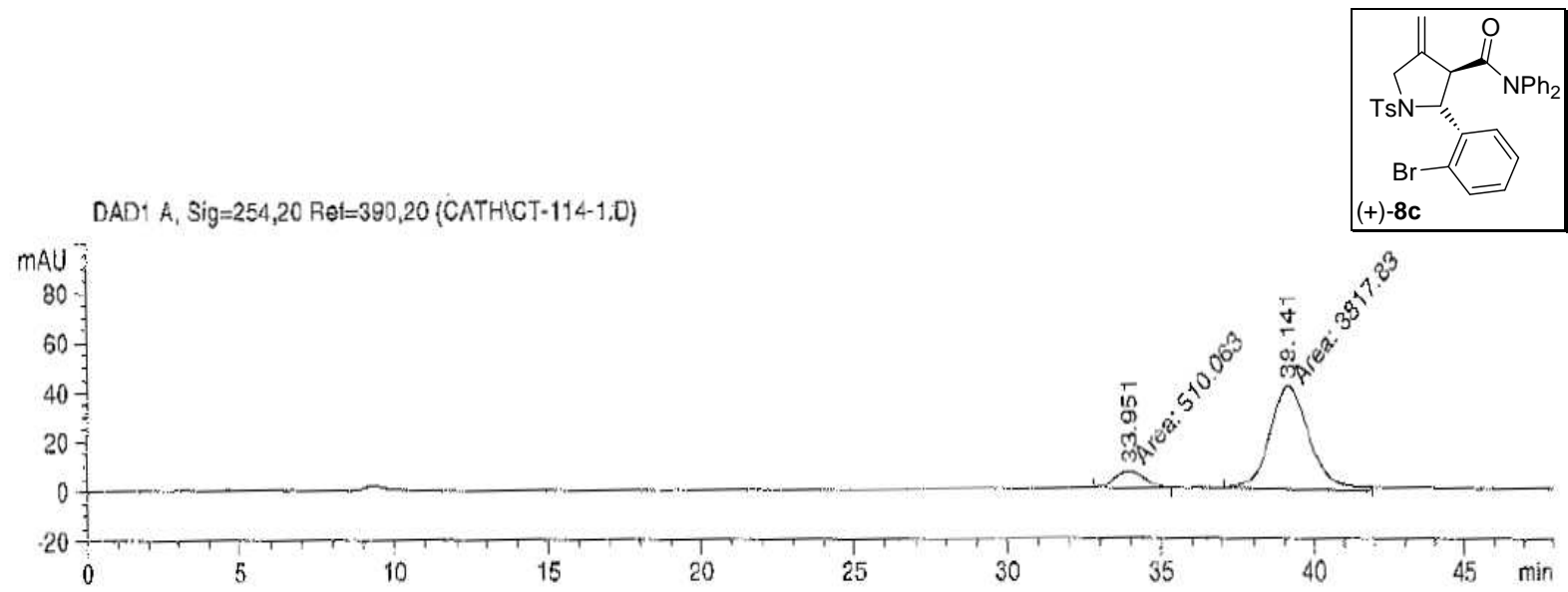

Signal 1: DAD1 A, $s i g=254,20$ Ref $=390,20$

\begin{tabular}{|c|c|c|c|c|c|}
\hline $\begin{array}{c}\text { Peak } \\
\#\end{array}$ & $\begin{array}{l}\text { RetTime Type } \\
\text { [min] }\end{array}$ & $\begin{array}{l}\text { widith } \\
\text { [min] }\end{array}$ & $\begin{array}{c}\text { Area } \\
{[m A J * s]}\end{array}$ & $\begin{array}{l}\text { Height } \\
\text { [mAU] }\end{array}$ & $\begin{array}{l}\text { Area } \\
\frac{8}{6}\end{array}$ \\
\hline 1 & $\mathrm{MM}$ & 1.2062 & 510 & 76 & \\
\hline 2 & & 1.5107 & 3817.82959 & 42.12120 & 88.2145 \\
\hline & & & 4327.8927 & 49.16896 & \\
\hline
\end{tabular}


Chiralcel OD-H column, 90:10 Hexanes:i-PrOH, $1 \mathrm{~mL} / \mathrm{min}, 30{ }^{\circ} \mathrm{C}$.

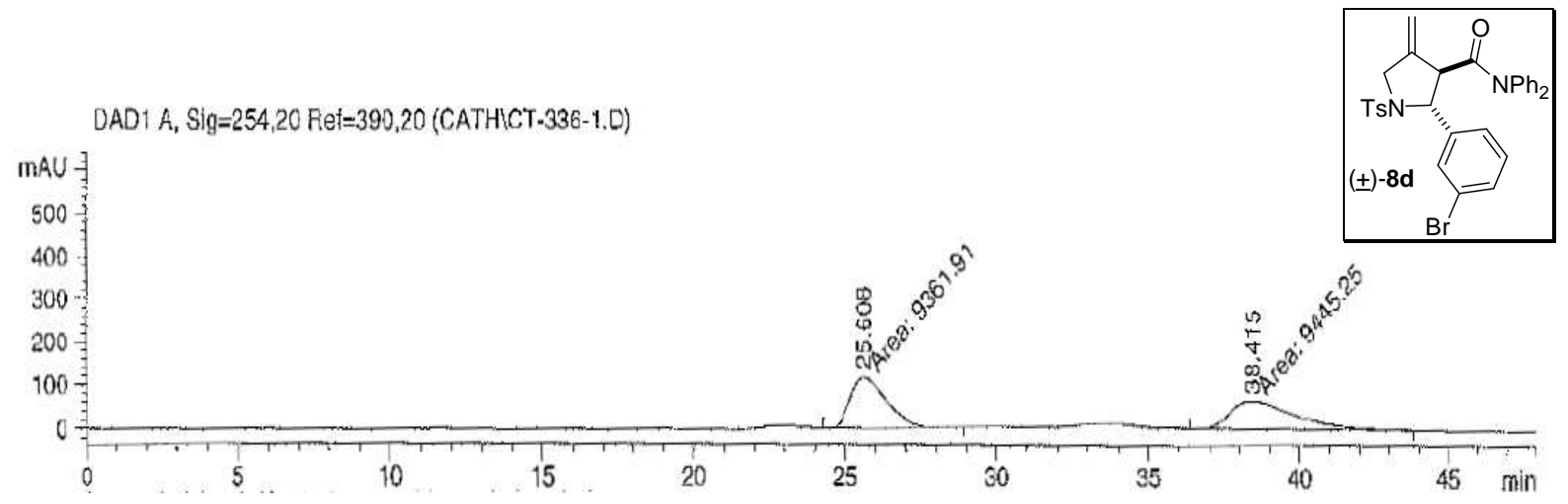

Signal 1: DAD1 A, $S i g=254,20$ Ref $=390,20$

\begin{tabular}{|c|c|c|c|c|c|}
\hline $\begin{array}{c}\text { Peak } \\
\#\end{array}$ & $\begin{array}{l}\text { RetTime Type } \\
\text { [min] }\end{array}$ & $\begin{array}{l}\text { width } \\
\text { [min] }\end{array}$ & $\begin{array}{c}\text { Area } \\
\text { [mAU*s] }\end{array}$ & $\begin{array}{l}\text { Height } \\
\text { [MAU] }\end{array}$ & $\begin{array}{c}\text { Area } \\
\text { \% }\end{array}$ \\
\hline 1 & $25.608 \mathrm{MM}$ & 1.3872 & 9361.91016 & 112.47778 & 49.7784 \\
\hline 2 & $38.415 \mathrm{MM}$ & 2.5236 & 9445.25098 & 62.37968 & 50.2216 \\
\hline Tota & Ls: & & $1.88072 \mathrm{e} 4$ & 174.85746 & \\
\hline
\end{tabular}

DAD1 A, Sig=254,20 Ref $=390,20$ (CATHICT-338-1.D)

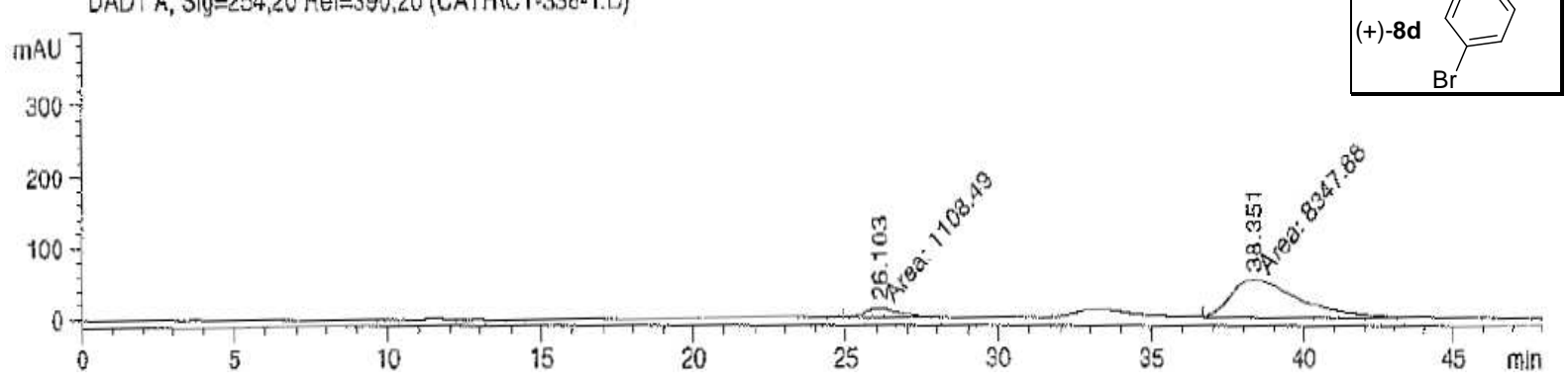

Signal 1: DAD1 A, Sig $=254,20$ Ref $=390,20$

\begin{tabular}{|c|c|c|c|c|c|}
\hline $\begin{array}{c}\text { Peak } \\
\#\end{array}$ & $\begin{array}{l}\text { RetTime Type } \\
\text { [min] }\end{array}$ & $\begin{array}{l}\text { Width } \\
\text { [min] }\end{array}$ & $\begin{array}{c}\text { Area } \\
{[m \Delta U * s]}\end{array}$ & $\begin{array}{l}\text { Height } \\
\text { [mAU] }\end{array}$ & $\begin{array}{c}\text { Area } \\
\frac{g}{6}\end{array}$ \\
\hline & & & & & \\
\hline 1 & $26.103 \mathrm{MM}$ & 1.3439 & 1108.48901 & 13.74700 & 31.7221 \\
\hline 2 & $38.351 \mathrm{MM}$ & 2.6159 & 8347.88086 & 53.18645 & 88.2779 \\
\hline t & & & 9456.36987 & 93345 & \\
\hline
\end{tabular}


Chiralpak AD column, 90:10 Hexanes:i-PrOH, $1 \mathrm{~mL} / \mathrm{min}, 30{ }^{\circ} \mathrm{C}$.

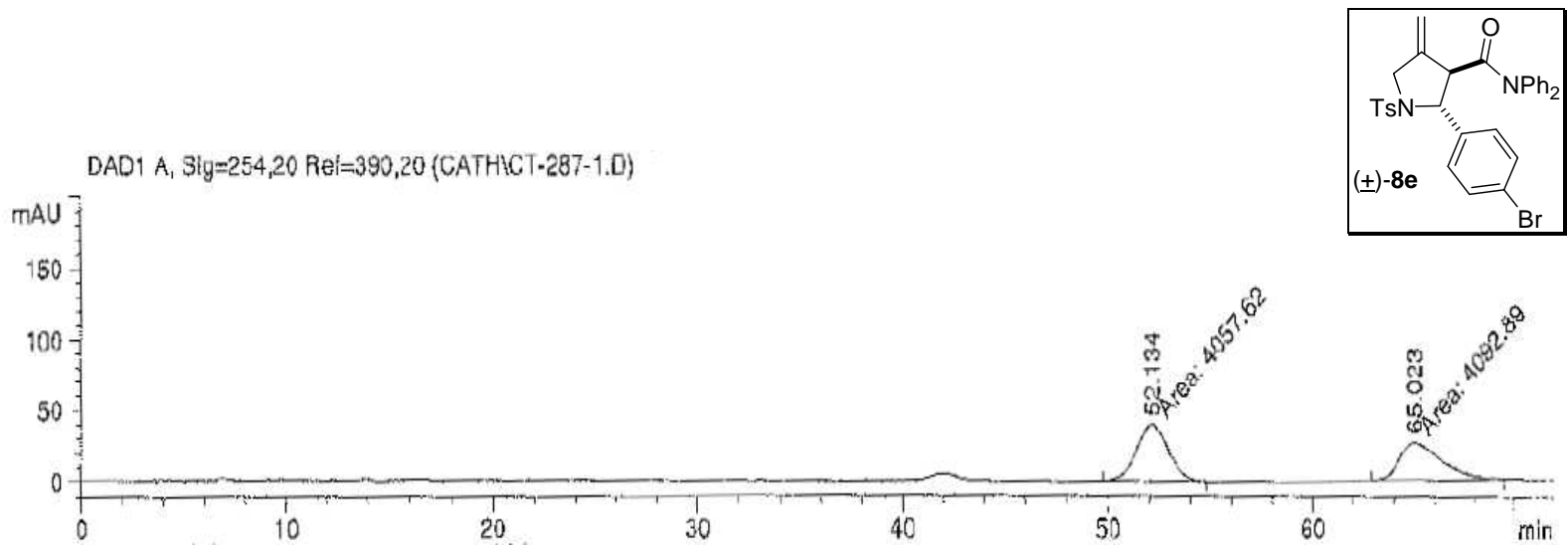

Signa1 1: $D A D 1 A, \quad S i g=254,20$ Ref $=390,20$

\begin{tabular}{|c|c|c|c|c|c|c|}
\hline $\begin{array}{c}\text { Peak } \\
\#\end{array}$ & $\begin{array}{c}\text { RetTime } \\
\text { [min] }\end{array}$ & Type & $\begin{array}{l}\text { width } \\
{[\mathrm{min}]}\end{array}$ & $\begin{array}{c}\text { Area } \\
{\left[\mathrm{mAJ}^{*} \in\right]}\end{array}$ & $\begin{array}{l}\text { Height } \\
\text { [mAU] }\end{array}$ & $\begin{array}{c}\text { Area } \\
\frac{8}{6}\end{array}$ \\
\hline 1 & 52.134 & MM & 1.7 & 4057.62451 & 38 & 36 \\
\hline 2 & 65.023 & MM & 2.5819 & 4092.89282 & 26.42012 & 50.2164 \\
\hline tal & & & & 8150.51733 & 65.03620 & \\
\hline
\end{tabular}

DAD1 A, Sig $=254,20$ Ree $=390,20$ (CATHICT-284-1,D)

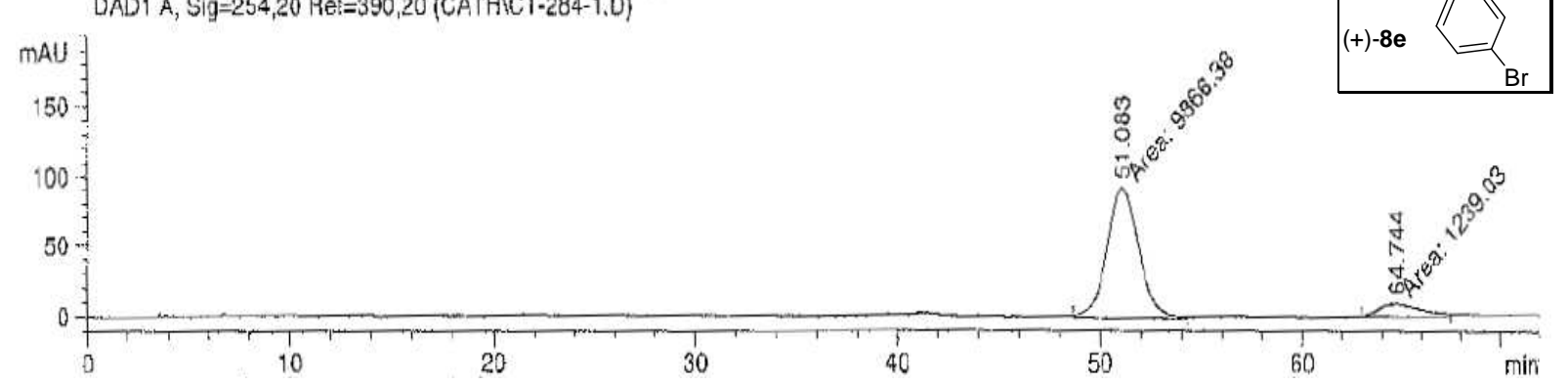

Signal 1: DAD1 A, $\operatorname{Sig}=254,20$ Ref $=390,20$

\begin{tabular}{|c|c|c|c|c|c|}
\hline $\begin{array}{c}\text { Peak } \\
\#\end{array}$ & $\begin{array}{l}\text { RetTime Type } \\
\text { [min] }\end{array}$ & $\begin{array}{l}\text { Width } \\
\text { [min] }\end{array}$ & $\begin{array}{c}\text { Area } \\
{\left[\mathrm{mAU}^{2} \mathrm{~s}\right]}\end{array}$ & $\begin{array}{l}\text { Height } \\
\text { [mAU] }\end{array}$ & $\begin{array}{c}\text { Area } \\
\frac{2}{6}\end{array}$ \\
\hline 1 & $\mathrm{MM}$ & & 9866 & & 88 \\
\hline 2 & $61.744 \mathrm{MM}$ & 2 & 1239.02747 & 8.19950 & 11.1570 \\
\hline & & & $11054 \mathrm{e}$ & 100.34516 & \\
\hline
\end{tabular}


Chiralpak AD column, 95:05 Hexanes:i-PrOH, $1 \mathrm{~mL} / \mathrm{min}, 30{ }^{\circ} \mathrm{C}$.

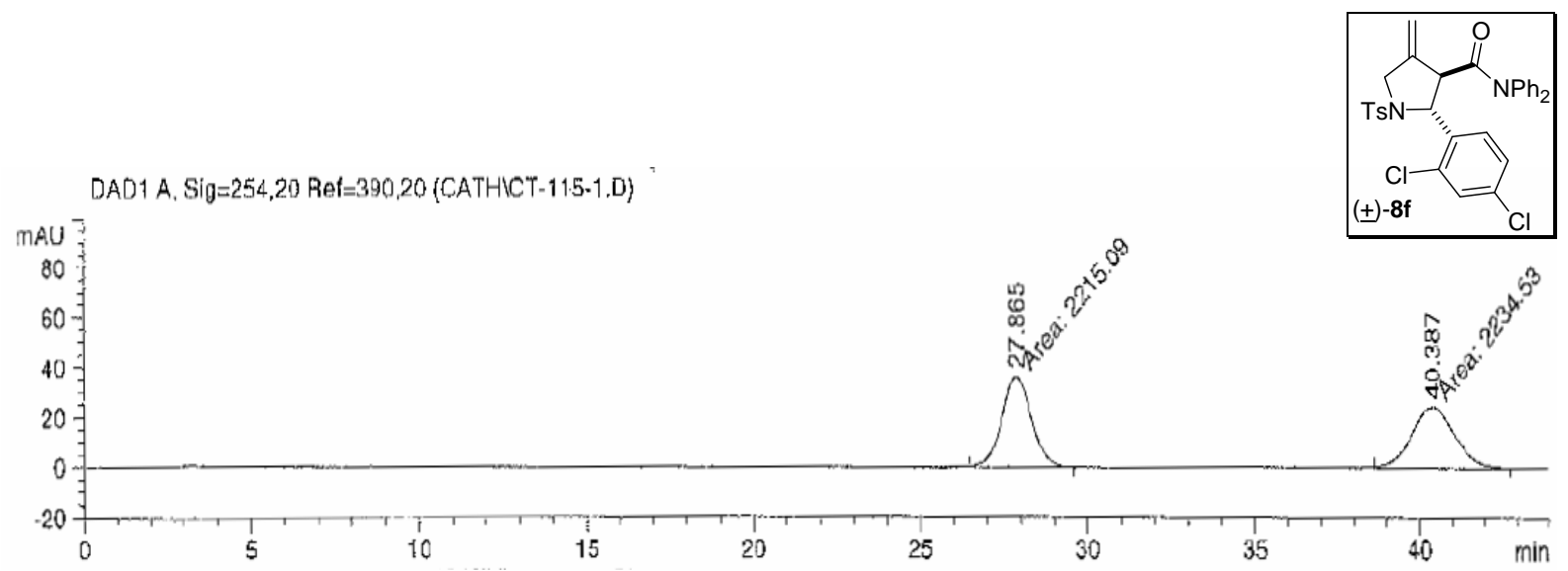

Signal 1: DAD1 A, $S i g=254,20$ Ref $=390,20$

\begin{tabular}{|c|c|c|c|c|c|}
\hline $\begin{array}{c}\text { Peak } \\
\#\end{array}$ & $\begin{array}{l}\text { RetTime Type } \\
\text { [min] }\end{array}$ & $\begin{array}{l}\text { width } \\
\text { [min] }\end{array}$ & $\begin{array}{c}\text { Area } \\
{\left[\mathrm{mAU} U^{*} \mathrm{~s}\right]}\end{array}$ & $\begin{array}{l}\text { Height } \\
\text { [mAU] }\end{array}$ & $\begin{array}{c}\text { Area } \\
\text { g }\end{array}$ \\
\hline-1 & - & & & & $-\cdots-\infty$ \\
\hline $\begin{array}{l}1 \\
2\end{array}$ & $\begin{array}{l}27.865 \mathrm{MM} \\
40.387 \mathrm{MM}\end{array}$ & $\begin{array}{l}1.0228 \\
1.5201\end{array}$ & $\begin{array}{l}2215.09009 \\
2234.52563\end{array}$ & $\begin{array}{l}36.09549 \\
24.50014\end{array}$ & $\begin{array}{l}49.7816 \\
50.2184\end{array}$ \\
\hline Total & & & 4449.61572 & 60.59563 & \\
\hline
\end{tabular}

DAD1 A, $\$$ ig $=254,20$ Ref $=390,20$ (CATHICT $\cdot 124-1 . D)$
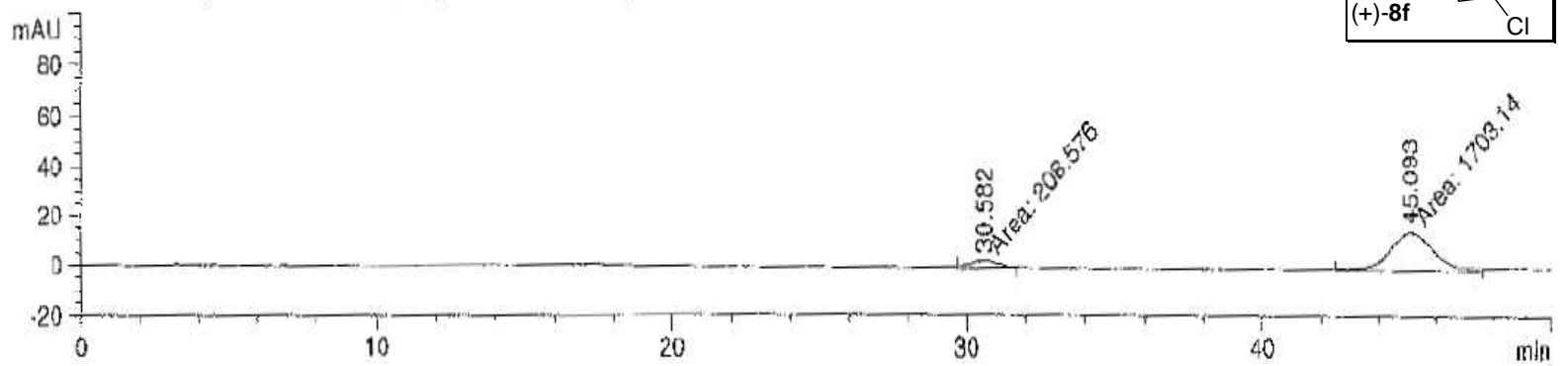

Signal 1: DAD1 A, Sig=254,20 Ref $=390,20$

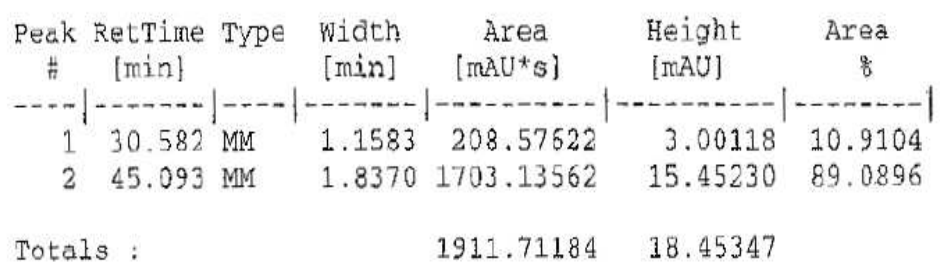




\section{Chiralpak AD column, 90:10 Hexanes:i-PrOH, $1 \mathrm{~mL} / \mathrm{min}, 30{ }^{\circ} \mathrm{C}$.}

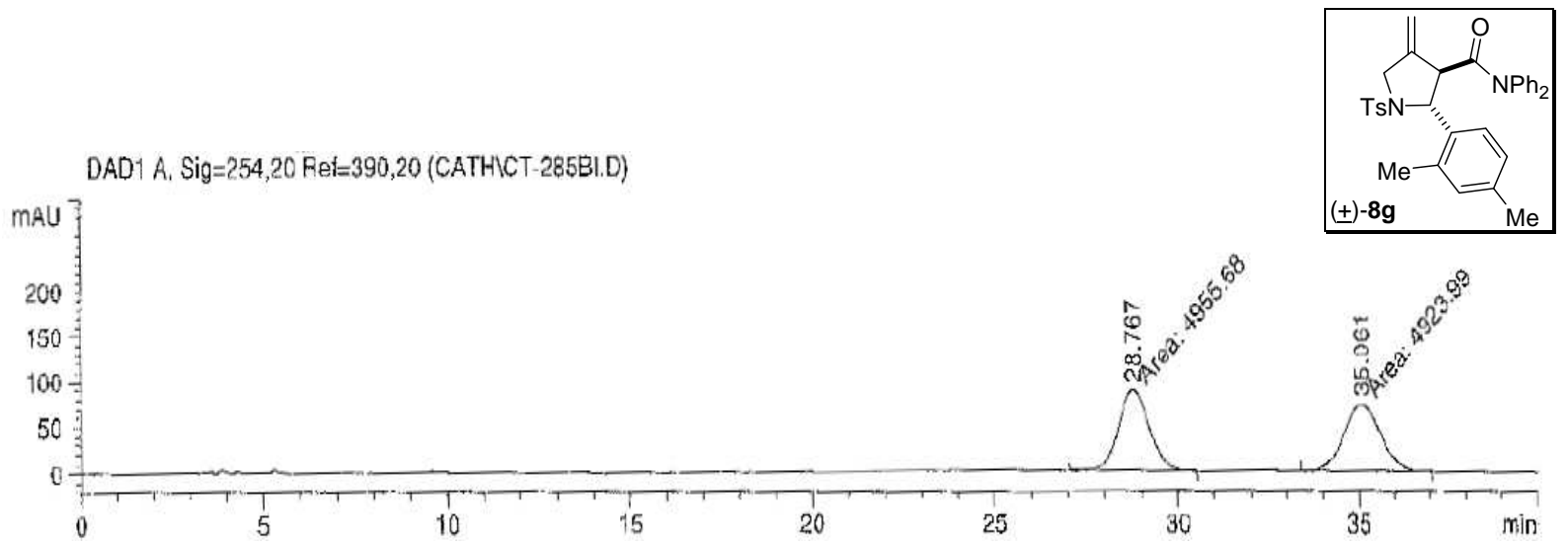

Signal 1: DADI A, Sig=254, 20 Ref $=390,20$

\begin{tabular}{|c|c|c|c|c|c|}
\hline $\begin{array}{c}\text { Peak } \\
\#\end{array}$ & $\begin{array}{l}\text { RetTime Type } \\
\text { [min] }\end{array}$ & $\begin{array}{l}\text { Width } \\
\text { [min] }\end{array}$ & $\begin{array}{c}\text { Area } \\
{\left[\mathrm{mAU}^{+} \mathrm{s}\right]}\end{array}$ & $\begin{array}{l}\text { Height } \\
\text { [MAU] }\end{array}$ & $\begin{array}{c}\text { Area } \\
\text { \% }\end{array}$ \\
\hline 1 & $28.767 \mathrm{MM}$ & 0.9592 & 4955.67627 & 86.10986 & 604 \\
\hline 2 & $35.061 \mathrm{MM}$ & 1.1601 & 4923.98682 & 70.74265 & 49.8396 \\
\hline Total & & & 9879.66309 & 156.85251 & \\
\hline
\end{tabular}

DAD1 A, Sig $=254,20$ Rel $=390,20$ (CATHCT 2828 BI.D)

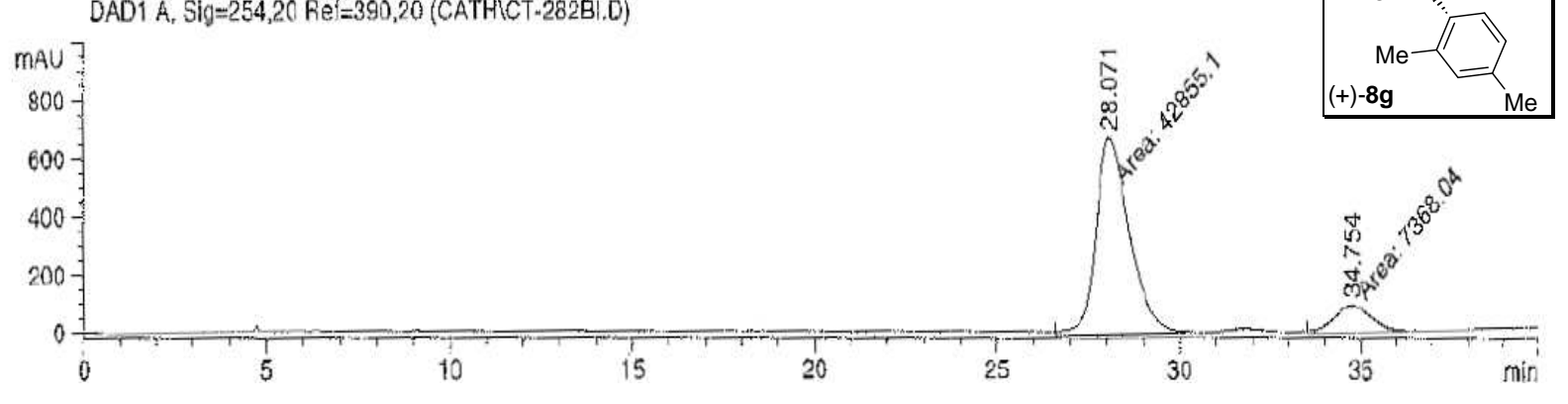

Signal 1: DAD1 A, Sig=254,20 Ref $=390,20$

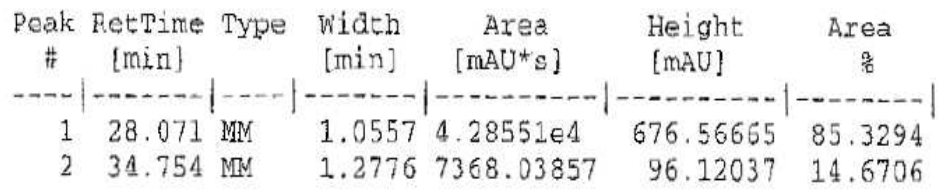

Totals: $\quad 5.02232 \mathrm{e} 4 \quad 772.68702$ 
Chiralcel OD-H column, 90:10 Hexanes:i-PrOH, $1 \mathrm{~mL} / \mathrm{min}, 30{ }^{\circ} \mathrm{C}$.

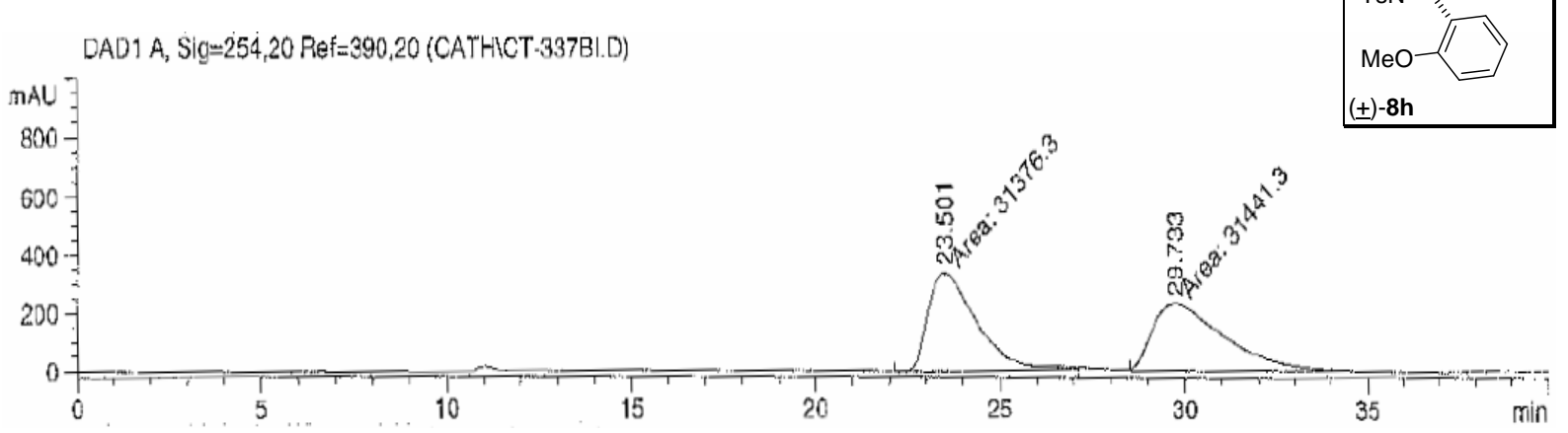

Signal 1: DADI A, $S i g=254,20$ Ref $=390,20$

\begin{tabular}{|c|c|c|c|c|c|}
\hline $\begin{array}{c}\text { Peak } \\
\#\end{array}$ & $\begin{array}{l}\text { RetTime Type } \\
\text { [nin] }\end{array}$ & $\begin{array}{l}\text { Width } \\
\text { [min] }\end{array}$ & $\begin{array}{c}\text { Area } \\
{\left[\mathrm{mAU}^{*} \&\right]}\end{array}$ & $\begin{array}{l}\text { Height } \\
\text { [mAU] }\end{array}$ & $\begin{array}{c}\text { Area } \\
\frac{\%}{6}\end{array}$ \\
\hline 1 & $23.501 \mathrm{MM}$ & 1.5372 & $3.13763 \mathrm{e} 4$ & 340.19492 & 49.9483 \\
\hline 2 & $29.733 \mathrm{MM}$ & 2.2442 & $3.14413 \mathrm{e} 4$ & 233.50130 & 50.0517 \\
\hline Tot: & & & 6.2817604 & 573.69621 & \\
\hline
\end{tabular}

DAD1 A, Stg=254,20 Reil=390,20 (CATHKCT-337-1.D)

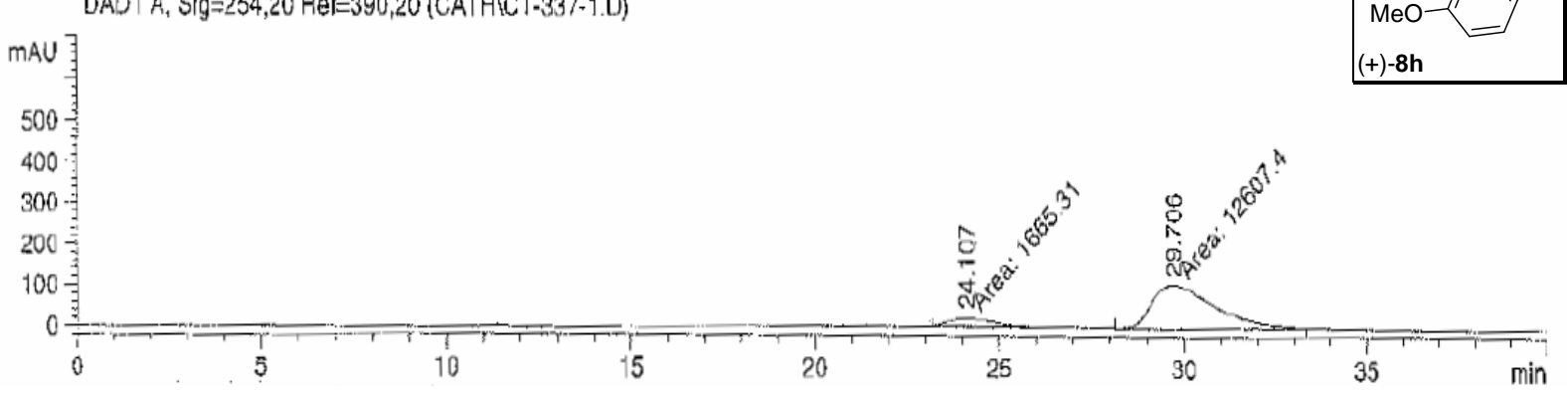

Signal 1: DAD1 A, Sig=254,20 Ref=390,20

\begin{tabular}{|c|c|c|c|c|c|}
\hline eak & $\begin{array}{l}\text { RetTime Type } \\
\text { [min] }\end{array}$ & $\begin{array}{l}\text { Width } \\
\text { [min] }\end{array}$ & $\begin{array}{c}\text { Area } \\
{\left[\mathrm{MAU}^{*} \mathrm{~s}\right]}\end{array}$ & $\begin{array}{l}\text { Height } \\
\text { [mAU] }\end{array}$ & $\begin{array}{c}\text { Area } \\
8\end{array}$ \\
\hline 1 & & & & & 11 \\
\hline 2 & & 1.9815 & $1.26074 \mathrm{e} 4$ & 106.04038 & 88.3322 \\
\hline & & & $1.42727 \mathrm{e} 4$ & 128.67183 & \\
\hline
\end{tabular}




\section{Chiralcel OD-H column, 90:10 Hexanes:i-PrOH, $1 \mathrm{~mL} / \mathrm{min}, 30{ }^{\circ} \mathrm{C}$.}

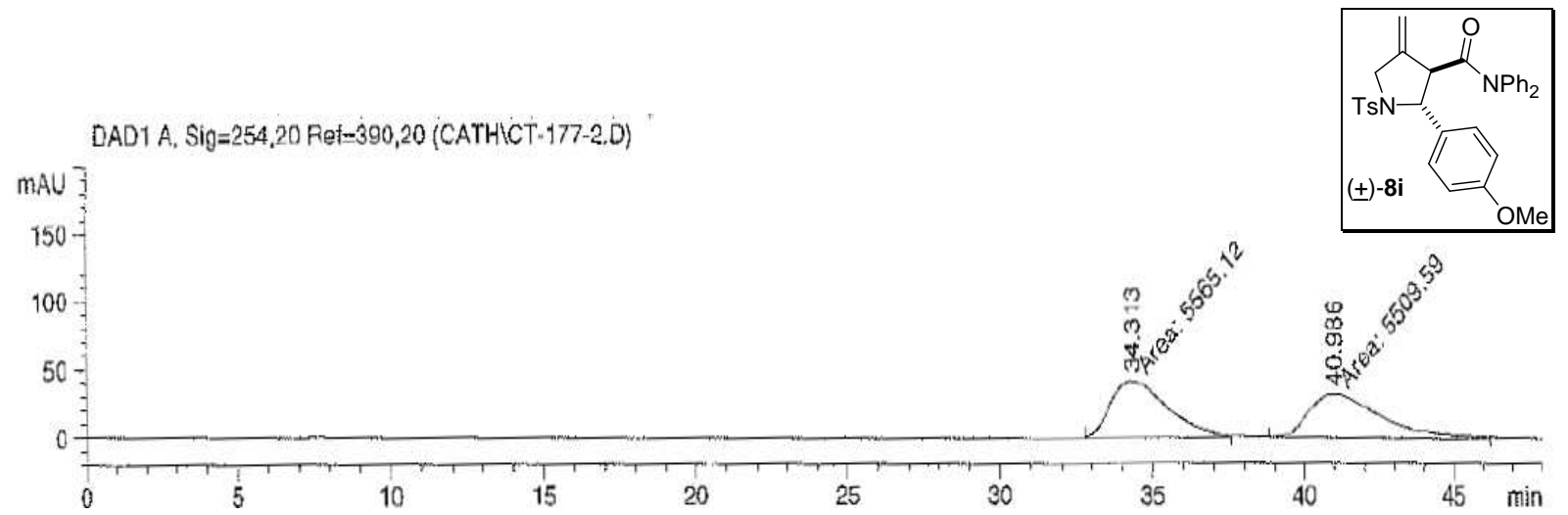

Signal 1: DAD1 A, Sig $=254,20$ Ref $=390,20$

\begin{tabular}{c|ccccc}
$\begin{array}{c}\text { Peak RetTime Type Width } \\
\text { \# } \\
\text { [min] }\end{array}$ & $\begin{array}{c}\text { Area } \\
\text { [min] }\end{array}$ & $\begin{array}{l}\text { Height } \\
\text { [mAU*s] }\end{array}$ & $\begin{array}{c}\text { Area } \\
\text { [mAU] }\end{array}$ & $\%$ \\
\hline 1 & $34.313 \mathrm{MM}$ & 2.1782 & 5565.12109 & 42.58122 & 50.2507 \\
2 & $40.986 \mathrm{MM}$ & 2.8204 & 5509.59229 & 32.55767 & 49.7493
\end{tabular}

Totals: $\quad 1.10747 \mathrm{e} 4 \quad 75.13889$

DAD 1 A, SIg $=254,20$ Fef $=390,20$ (CATHICT-187-1.D)

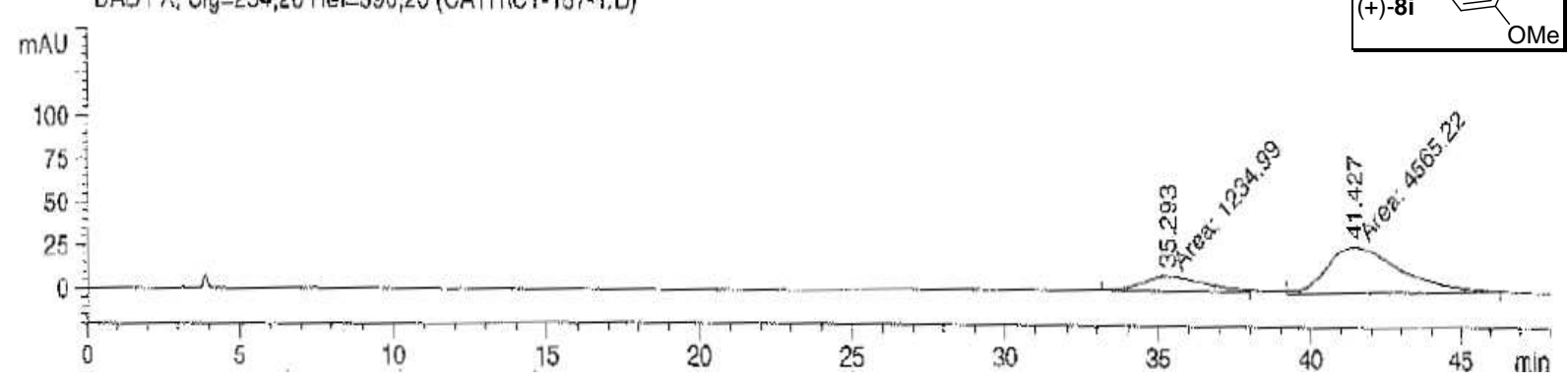

Signal 1: DAD1 A, Sig=254, 20 Ref $=390,20$

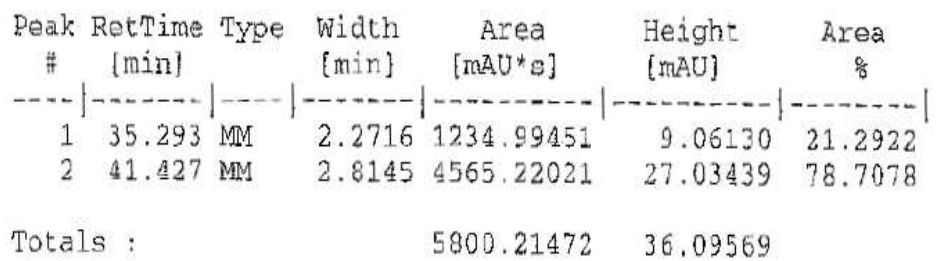


ChiralPak AD column, 85:15 Hexanes:i-PrOH, $1 \mathrm{~mL} / \mathrm{min}, 30{ }^{\circ} \mathrm{C}$.

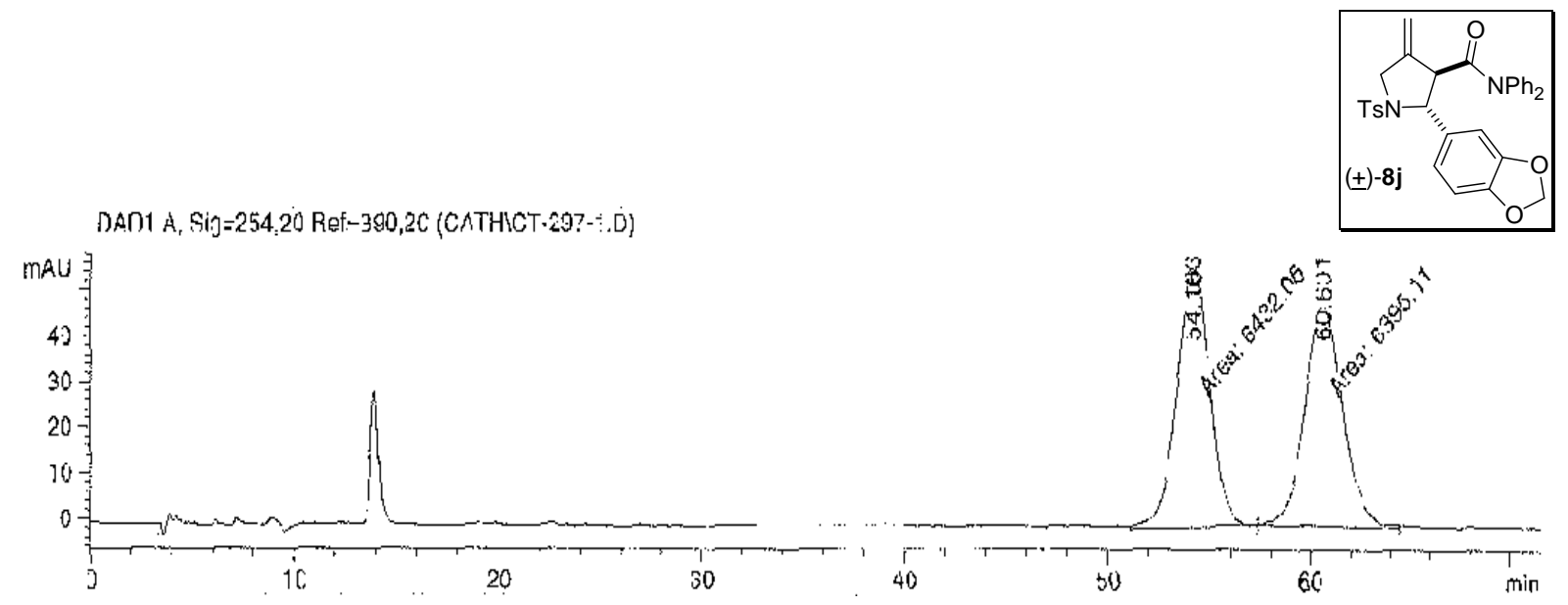

Signal i: DHL1 A, Sig $=254,20$ Ref $=390,20$

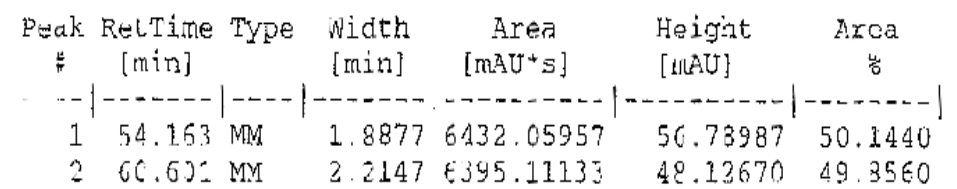

Totals : $\quad 1.2827294 \quad 104.91657$

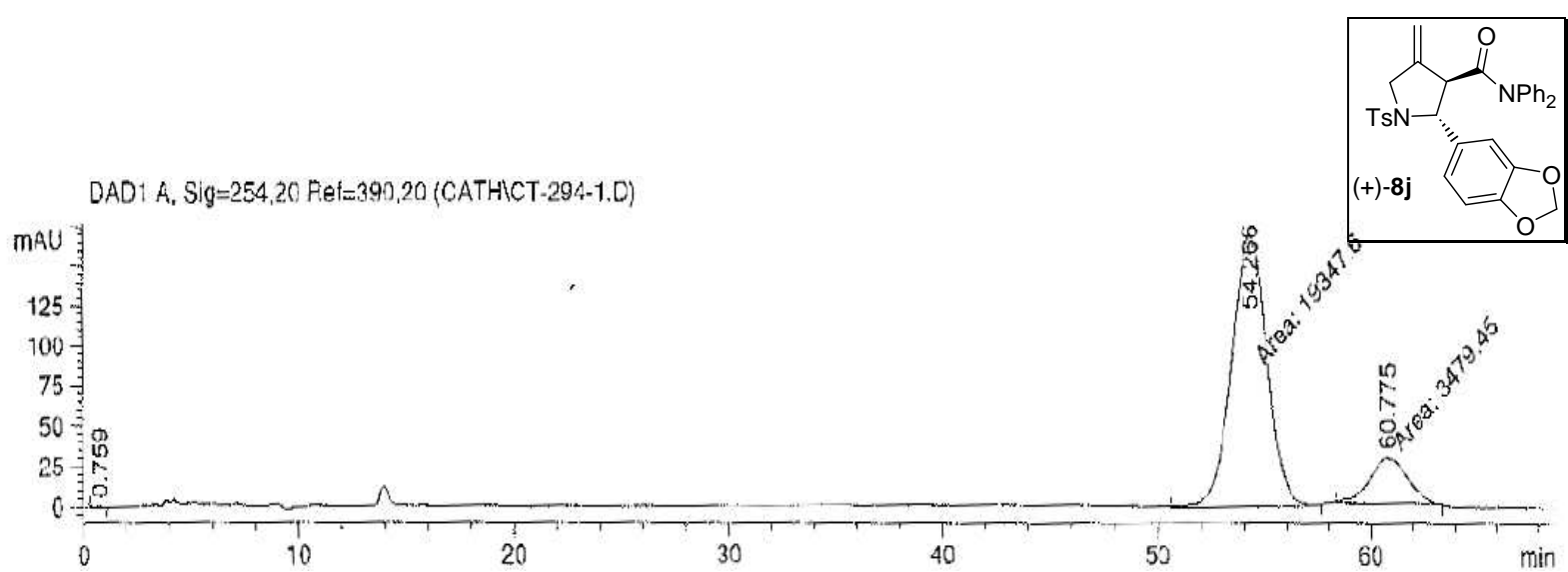

Signal 1: DAD1 A, $S i g=254,20$ Ref $=390,20$

\begin{tabular}{|c|c|c|c|c|c|}
\hline $\begin{array}{c}\text { Peak } \\
\quad \#\end{array}$ & $\begin{array}{l}\text { RetTime Type } \\
\text { [min] }\end{array}$ & $\begin{array}{l}\text { Width } \\
\text { [min] }\end{array}$ & $\begin{array}{c}\text { Area } \\
{[\mathrm{mAU} * \mathrm{~s}]}\end{array}$ & $\begin{array}{l}\text { Height } \\
\text { [mAU] }\end{array}$ & $\begin{array}{l}\text { Area } \\
\text { go }\end{array}$ \\
\hline 1 & $0.759 \mathrm{PV}$ & 0.3724 & 9.65342 & $3.50549 \mathrm{e}-1$ & 0.0423 \\
\hline 2 & $54.266 \mathrm{MM}$ & 1.9183 & $1.93476 \mathrm{e} 4$ & 168. & 84.7215 \\
\hline 3 & $60.775 \mathrm{MM}$ & 2.0675 & 3479.44678 & 28.04856 & 15.2362 \\
\hline & & & $2.28367 \mathrm{e} 4$ & 96.50171 & \\
\hline
\end{tabular}


Chiralpak AD column, 93:07 Hexanes:i-PrOH, $1 \mathrm{~mL} / \mathrm{min}, 30{ }^{\circ} \mathrm{C}$.

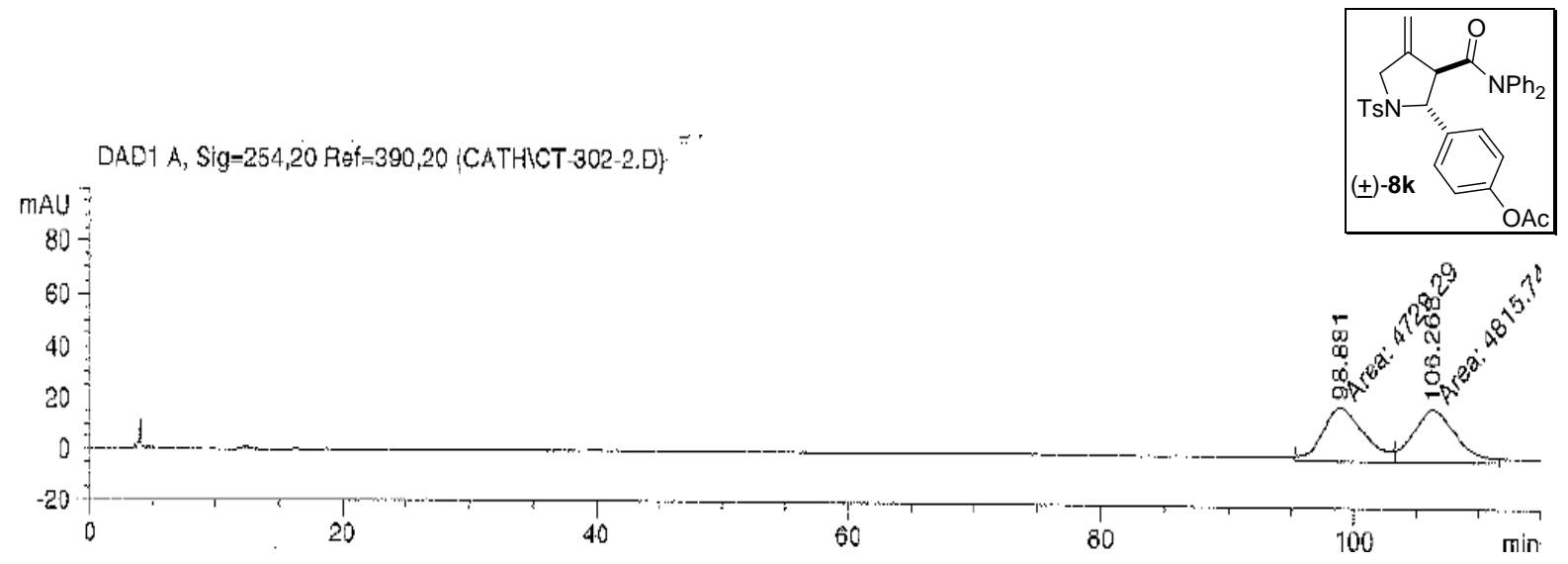

Signal 1: DAD1 A, $\operatorname{Sig}=254,20$ Ref $=390,20$

\begin{tabular}{|c|c|c|c|c|}
\hline $\begin{array}{l}\text { Peak RetTime Type } \\
\text { 茾 [min] }\end{array}$ & $\begin{array}{l}\text { Widith } \\
\text { [min] }\end{array}$ & $\begin{array}{c}\text { Area } \\
{\left[\mathrm{MAU} U^{\star} \mathrm{S}\right]}\end{array}$ & $\begin{array}{l}\text { Height } \\
{[\operatorname{mAU}]}\end{array}$ & $\begin{array}{c}\text { Area } \\
\%\end{array}$ \\
\hline & & & & \\
\hline $198.881 \mathrm{MF}$ & 3.8309 & 4729.29004 & 20.57533 & 471. \\
\hline $2106.268 \mathrm{EM}$ & 4 & 4815.74463 & 0.06341 & 50.4529 \\
\hline otals & & 9545.03467 & 873 & \\
\hline
\end{tabular}

DAD A A Sig=254,20 Rof $=390,20$ (CATHLCT-308-1,D)

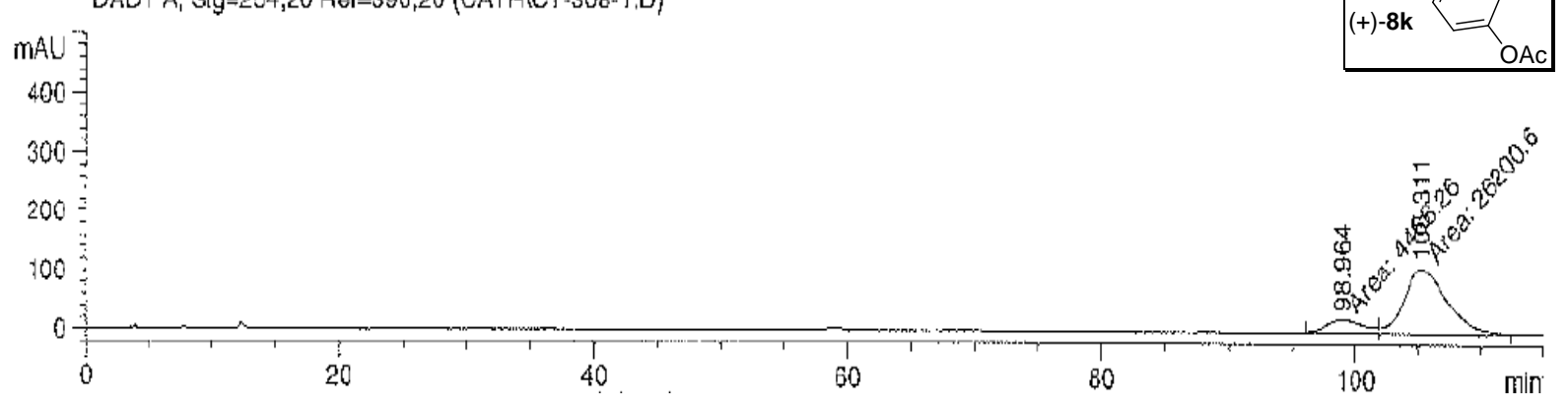

Signal 1: DAD1 A, Sig=254,20 Ref $=390,20$

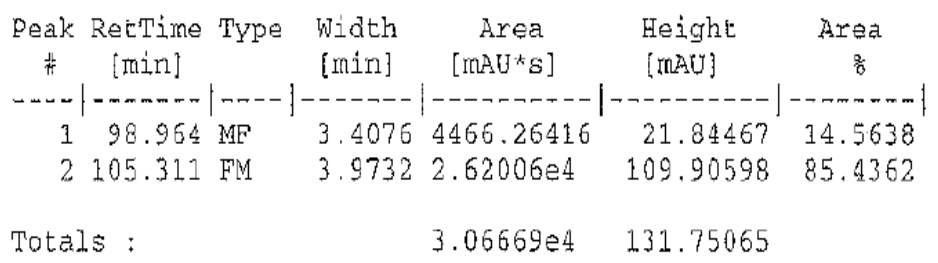


Chiralcel OD-H column, 90:10 Hexanes:i-PrOH, $1 \mathrm{~mL} / \mathrm{min}, 30{ }^{\circ} \mathrm{C}$.

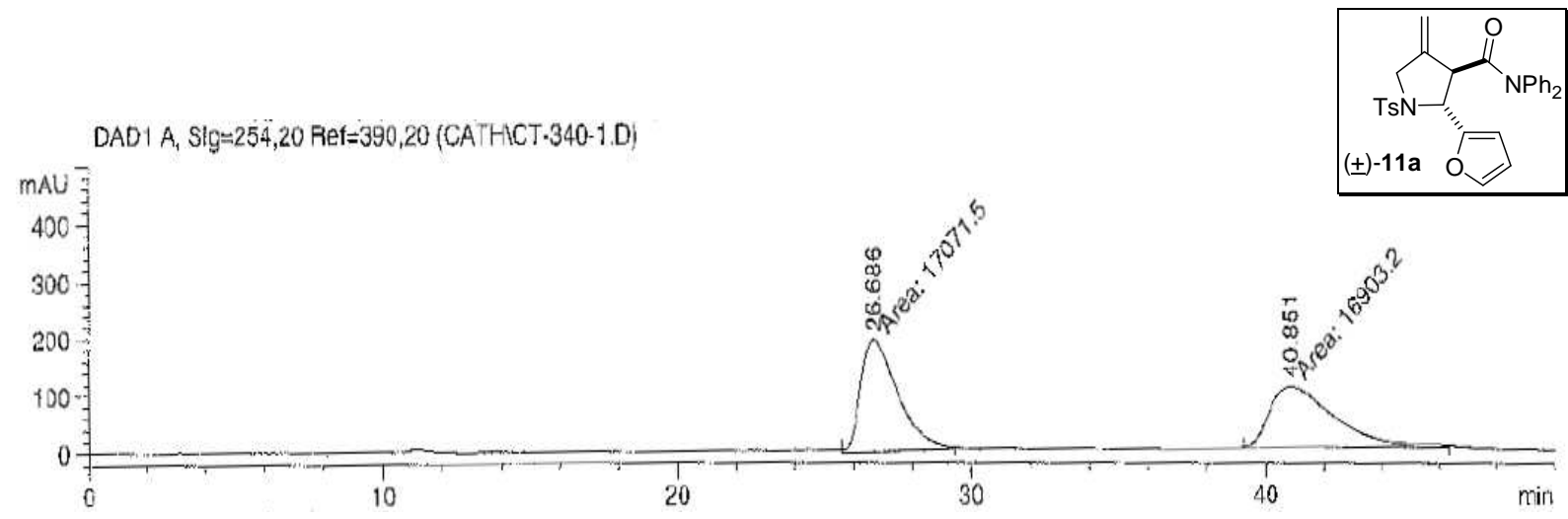

Signal 1: DAD1 A, Sig $=254,20$ Ref $=390,20$

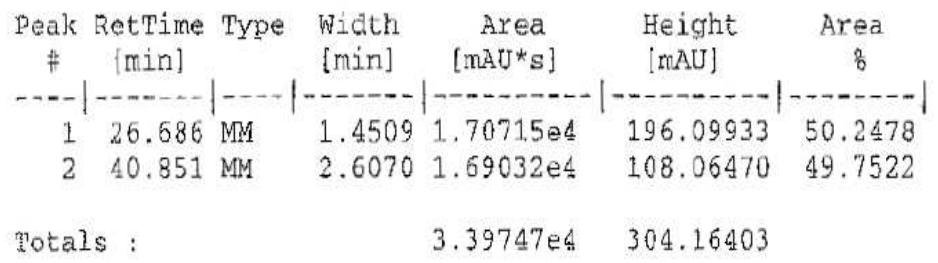

DAD1 A, Sig $=254,20$ Ref $=390,20$ (CATHICT-342-1.D)

$\mathrm{mAU}$
400
300
200
100
0

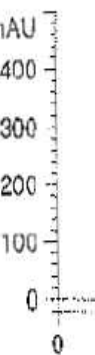

0

Signal 1: DAD1 A, Sig=254,20 Ref $=390,20$

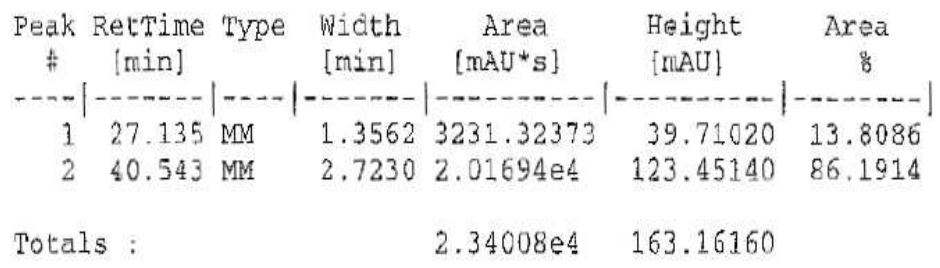


Chiralcel OD-H column, 90:10 Hexanes:i-PrOH, $1 \mathrm{~mL} / \mathrm{min}, 30{ }^{\circ} \mathrm{C}$.

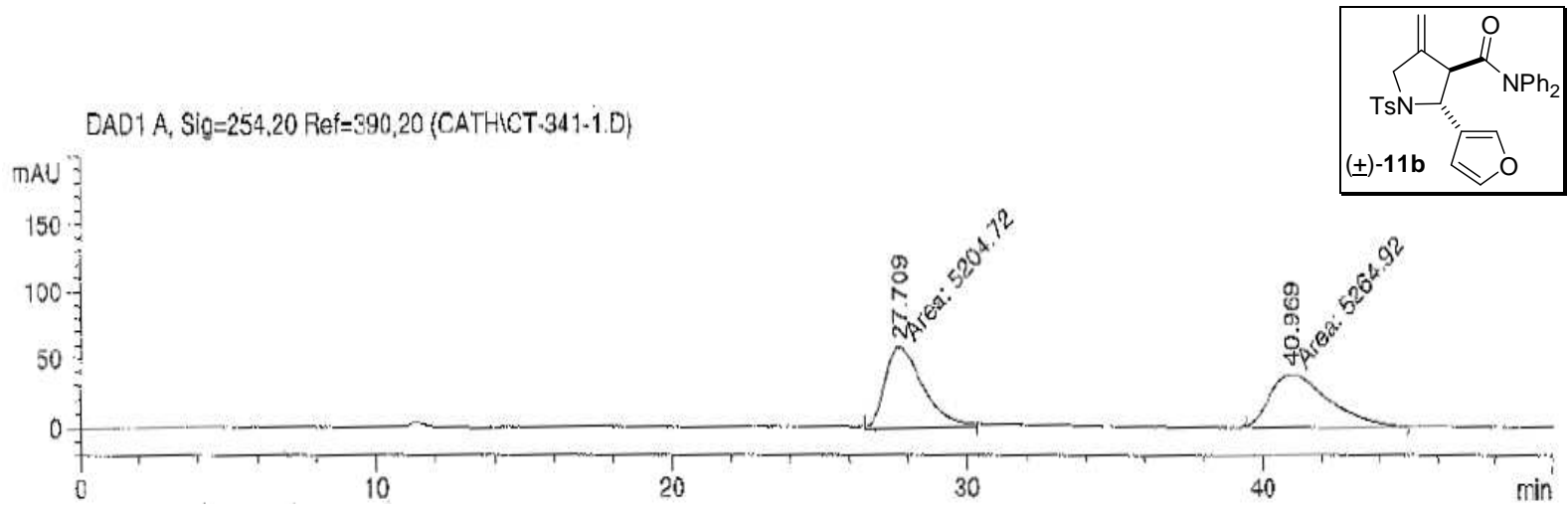

Signal 1: DAD1 A, Sig $=254,20$ Ref $=390,20$

\begin{tabular}{|c|c|c|c|c|c|}
\hline $\begin{array}{l}\text { Peak } \\
\text { \# }\end{array}$ & $\begin{array}{l}\text { RetTime Type } \\
\text { [min] }\end{array}$ & $\begin{array}{l}\text { Width } \\
{[\min ]}\end{array}$ & $\begin{array}{c}\text { Area } \\
{\left[\mathrm{mAU}^{*} \mathrm{~s}\right]}\end{array}$ & $\begin{array}{l}\text { Height } \\
\text { [mAU] }\end{array}$ & $\begin{array}{c}\text { Area } \\
\xi\end{array}$ \\
\hline 1 & 27 & & 5204 & & \\
\hline 2 & $40.969 \mathrm{MM}$ & 2.3504 & 5254.92285 & 37.33380 & 50.2875 \\
\hline To & & & $1.04696 \mathrm{e}$ & 95.58425 & \\
\hline
\end{tabular}

DAD1 $A$, Sig $=254,20$ Rei=390,20 (CATHICT-343-1.D)
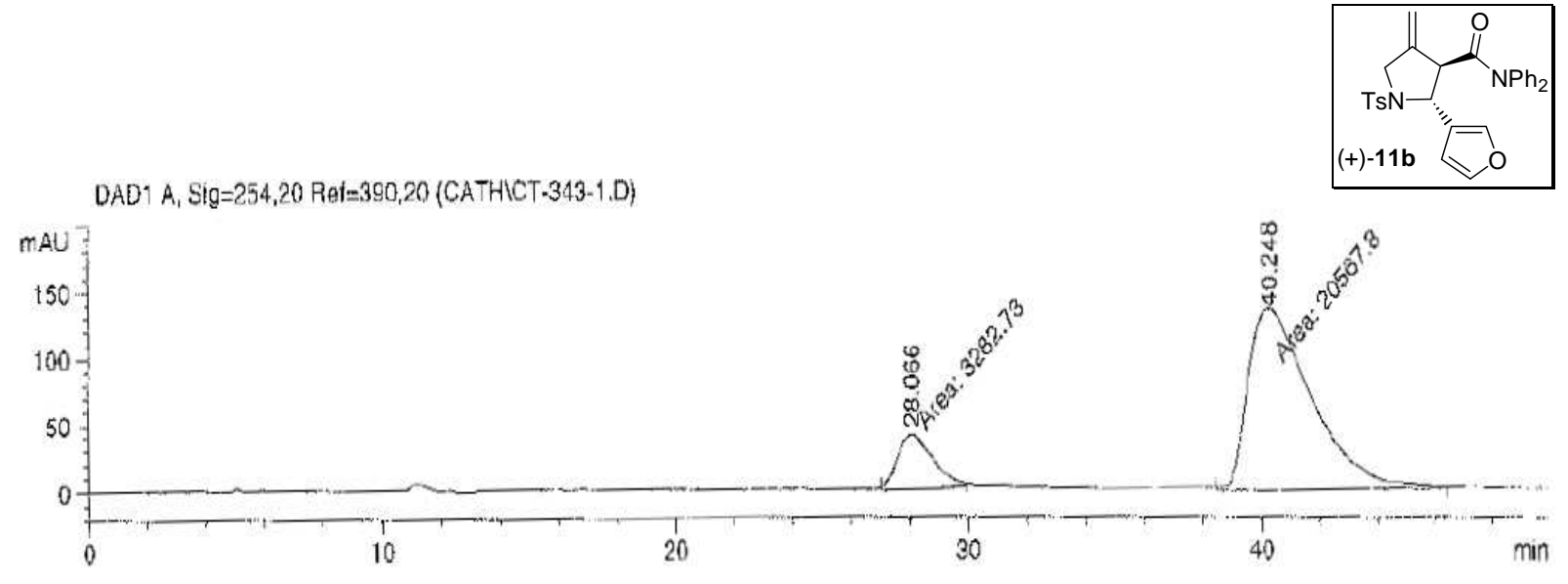

Signal 1: DAD1 $A, \operatorname{Sig}=254,20$ Ref $=390,20$

\begin{tabular}{|c|c|c|c|c|c|}
\hline $\begin{array}{c}\text { Peak } \\
\#\end{array}$ & $\begin{array}{l}\text { RetTime Type } \\
\text { [min] }\end{array}$ & $\begin{array}{l}\text { Width } \\
\text { [min] }\end{array}$ & $\begin{array}{c}\text { Area } \\
\text { [MAU*s] }\end{array}$ & $\begin{array}{l}\text { Height } \\
{[\mathrm{mAU}]}\end{array}$ & $\begin{array}{c}\text { Area } \\
\text { \% }\end{array}$ \\
\hline 1 & $28.066 \mathrm{Mv}$ & & 3282.72778 & & 7638 \\
\hline 2 & $40.248 \mathrm{MM}$ & 2.5215 & $2.05678 \mathrm{e} 4$ & 135.94855 & 86.2362 \\
\hline La & & & 2.3850504 & 175.04988 & \\
\hline
\end{tabular}


ChiralPak AD column, 85:15 hexane:i-PrOH, $1 \mathrm{~mL} / \mathrm{min}, 30{ }^{\circ} \mathrm{C}$.

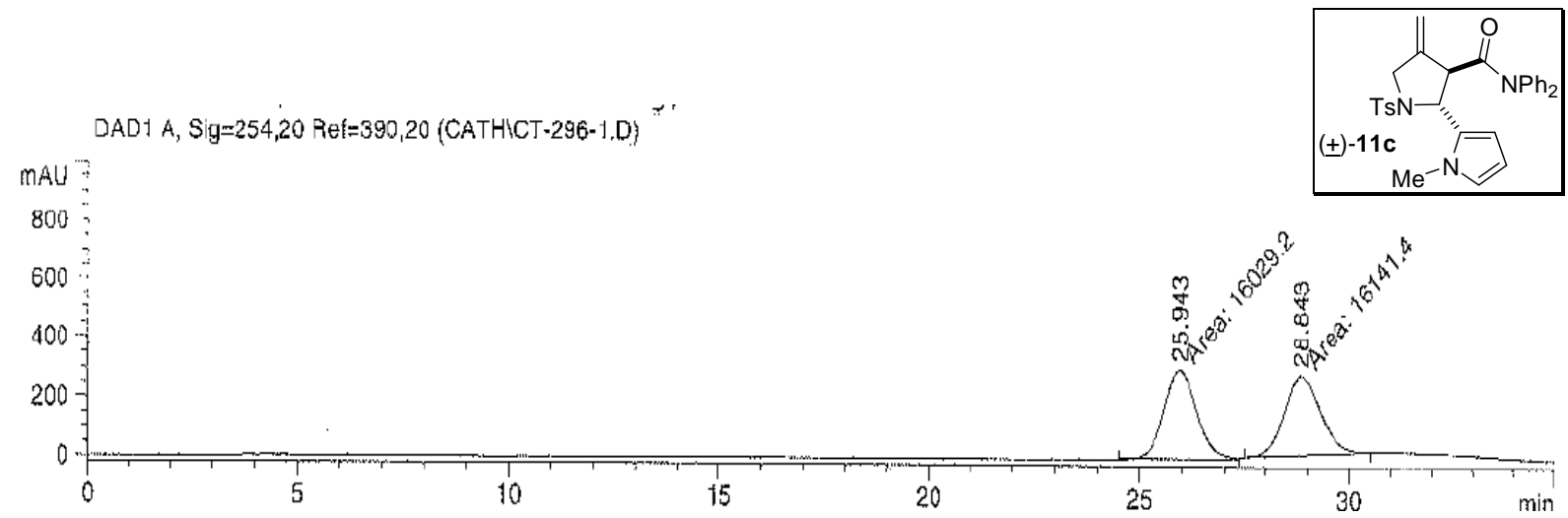

Signal 1: DAD1 A, $\$$ ig $=254,20$ Ref $=390,20$

\begin{tabular}{|c|c|c|c|c|c|}
\hline $\begin{array}{c}\text { Peak } \\
\text { \# }\end{array}$ & $\begin{array}{l}\text { RetTime Type } \\
\text { [min.] }\end{array}$ & $\begin{array}{l}\text { Width } \\
\text { [min] }\end{array}$ & $\begin{array}{c}\text { Area } \\
\text { [mAL*s] }\end{array}$ & $\begin{array}{l}\text { Helght } \\
\text { [mAU] }\end{array}$ & $\begin{array}{c}\text { Area } \\
\frac{\circ}{8}\end{array}$ \\
\hline & & & & & \\
\hline$\hat{1}$ & $\begin{array}{l}25.943 \mathrm{MM} \\
28.843 \mathrm{MM}\end{array}$ & 0.8932 & 1.602 & 299.08334 & \\
\hline 2 & & & 1.618 & 268.8 & 44 \\
\hline ta & & & 3.21 & 567 & \\
\hline
\end{tabular}

DAD1 A, Sig $=254,20$ Ref $=390,20$ (CATHCT-293-1.D)

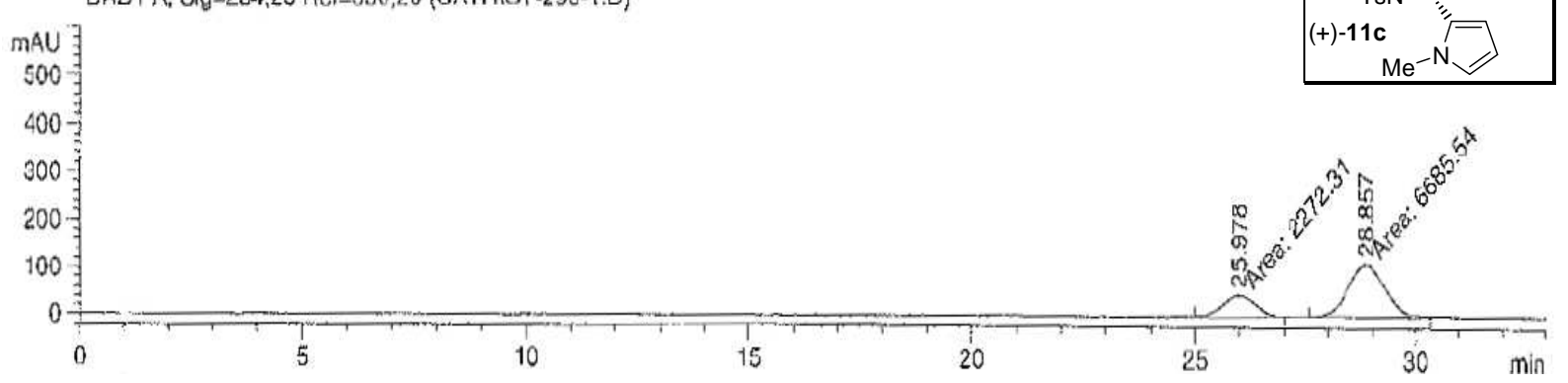

Signa1 1: DAD1 A, Sig=254,20 Ref=390,20

\begin{tabular}{|c|c|c|c|c|c|}
\hline $\begin{array}{c}\text { Peak } \\
\text { \# }\end{array}$ & $\begin{array}{l}\text { RetTime Type } \\
\text { [min] }\end{array}$ & $\begin{array}{l}\text { width } \\
\text { [min] }\end{array}$ & $\begin{array}{c}\text { Area } \\
{\left[\mathrm{MAU}^{*} \mathrm{~s}\right]}\end{array}$ & $\begin{array}{l}\text { Height } \\
\text { [MAU] }\end{array}$ & $\begin{array}{c}\text { Area } \\
8\end{array}$ \\
\hline 1 & $25.978 \mathrm{MM}$ & 0.8508 & - $20-10-1$ & 4300606 & 667 \\
\hline 2 & $28.857 \mathrm{MM}$ & 0.9985 & 6685.53711 & $\begin{array}{r}43.59000 \\
111.59783\end{array}$ & $\begin{array}{l}25.3067 \\
74.6333\end{array}$ \\
\hline otal & & & 8957.8 & 155 & \\
\hline
\end{tabular}


ChiralPak AD column, 80:20 hexane:i-PrOH, $1 \mathrm{~mL} / \mathrm{min}, 30{ }^{\circ} \mathrm{C}$.

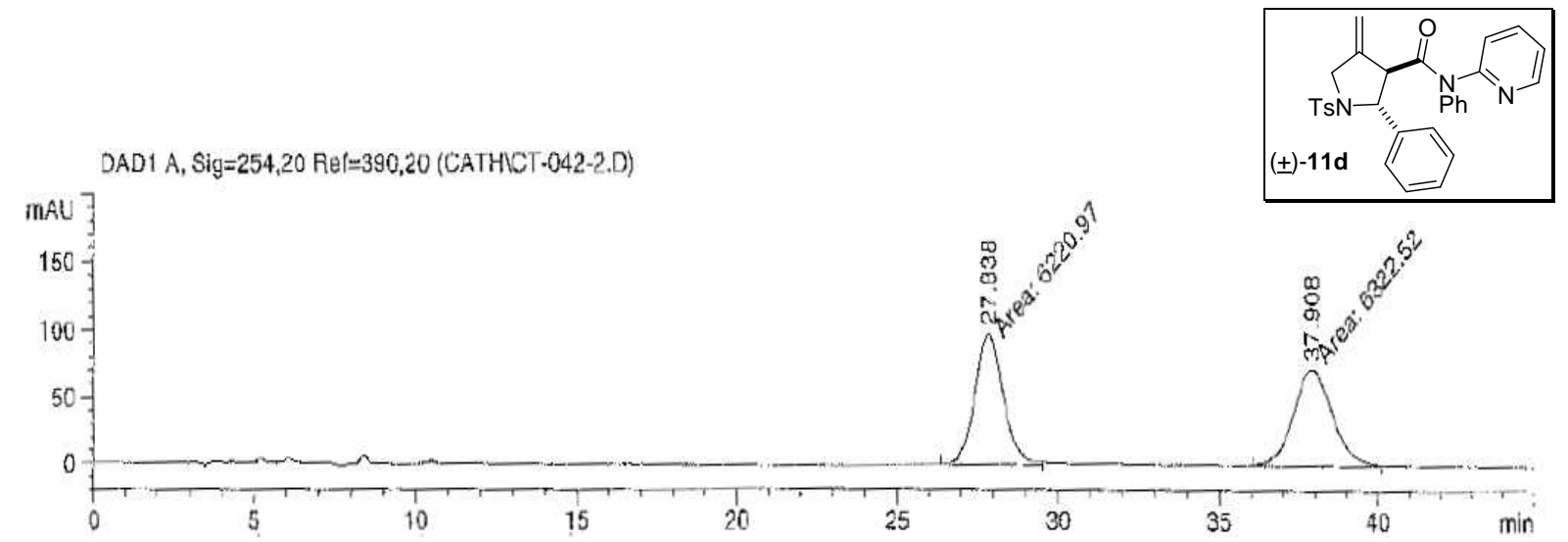

Signal 1: DAD1 A, $S i g=254,20$ Ref $=390,20$

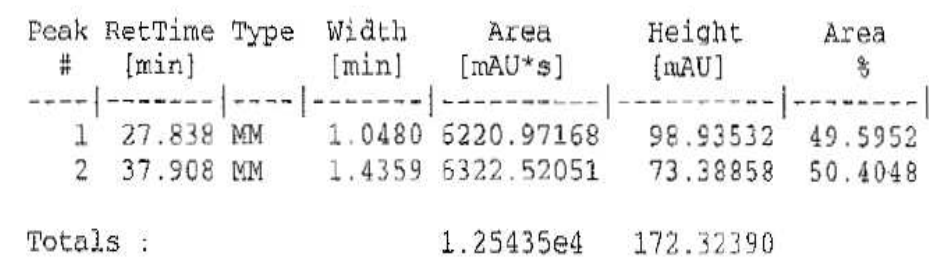

DAD1 A, Sig=254,20 Ref $=390,20$ (CATHICT-346-1.D)
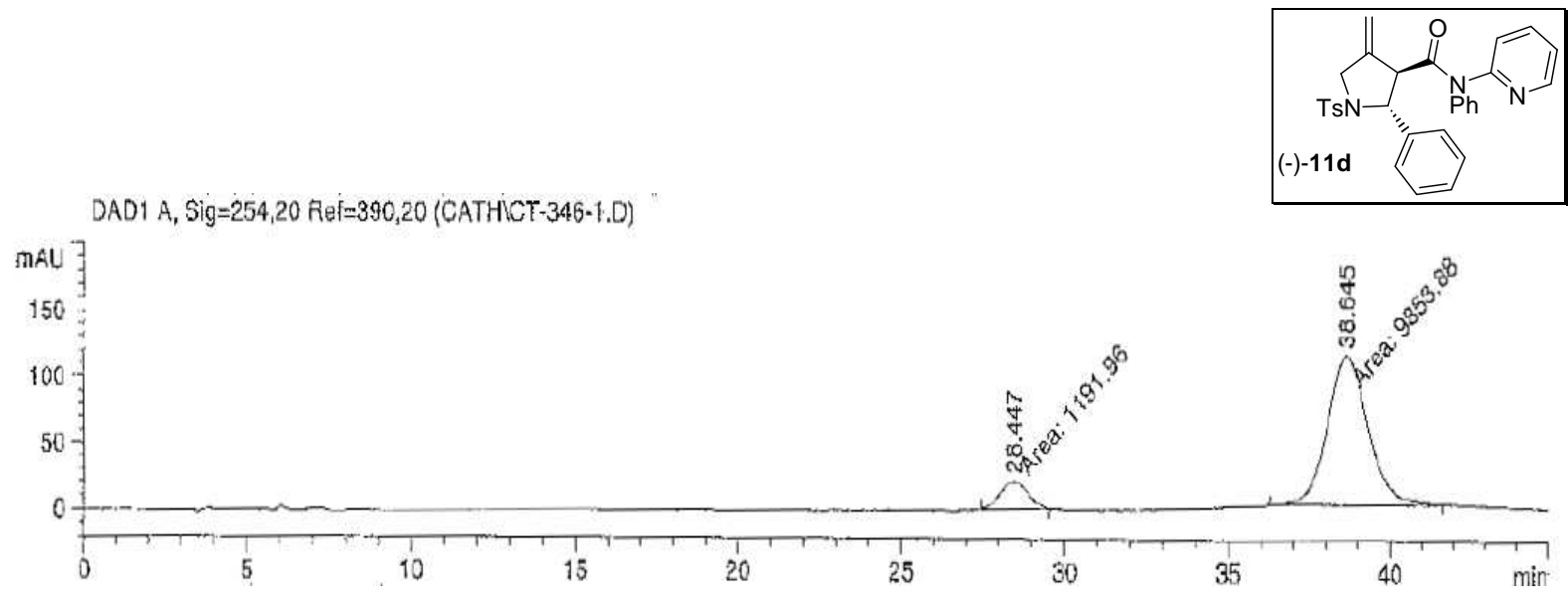

Signal 1: DAD1 A, Sig=254, 20 Ref $=390,20$

\begin{tabular}{|c|c|c|c|c|c|}
\hline $\begin{array}{c}\text { Peak } \\
\#\end{array}$ & $\begin{array}{l}\text { RetTime Type } \\
\text { [min] }\end{array}$ & $\begin{array}{l}\text { Width } \\
\text { [min] }\end{array}$ & $\begin{array}{c}\text { Area } \\
{\left[\mathrm{mAU}^{*} \mathrm{~s}\right]}\end{array}$ & $\begin{array}{l}\text { Height } \\
\text { \{mAU] }\end{array}$ & $\begin{array}{c}\text { Area } \\
\text { : }\end{array}$ \\
\hline 1 & $28.447 \mathrm{MM}$ & & 1191.95935 & 20.51290 & 10.7910 \\
\hline 2 & $38.645 \mathrm{mM}$ & 1. & 9853.87793 & 111.99243 & 89.2090 \\
\hline ted & & & $1.10458 \mathrm{e} 4$ & 132.50533 & \\
\hline
\end{tabular}

